\title{
Characterization of the airway epithelial bioelectric mechanisms associated with the effects of epithelium-derived relaxing factor in guinea pig-isolated trachea
}

\author{
Richard Alan Johnston \\ West Virginia University
}

Follow this and additional works at: https://researchrepository.wvu.edu/etd

\footnotetext{
Recommended Citation

Johnston, Richard Alan, "Characterization of the airway epithelial bioelectric mechanisms associated with the effects of epithelium-derived relaxing factor in guinea pig-isolated trachea" (2000). Graduate Theses, Dissertations, and Problem Reports. 1233.

https://researchrepository.wvu.edu/etd/1233

This Dissertation is protected by copyright and/or related rights. It has been brought to you by the The Research Repository @ WVU with permission from the rights-holder(s). You are free to use this Dissertation in any way that is permitted by the copyright and related rights legislation that applies to your use. For other uses you must obtain permission from the rights-holder(s) directly, unless additional rights are indicated by a Creative Commons license in the record and/ or on the work itself. This Dissertation has been accepted for inclusion in WVU Graduate Theses, Dissertations, and Problem Reports collection by an authorized administrator of The Research Repository @ WVU. For more information, please contact researchrepository@mail.wvu.edu.
} 


\title{
Characterization of the Airway Epithelial Bioelectric Mechanisms Associated with the Effects of Epithelium-Derived Relaxing Factor in Guinea-Pig Isolated Trachea
}

\author{
DISSERTATION \\ Submitted to the School of Medicine \\ at West Virginia University \\ In Partial Fulfillment of the Requirements \\ for the Degree of \\ Doctor of Philosophy \\ in Pharmacology and Toxicology \\ by \\ Richard A. Johnston \\ Jeffrey S. Fedan, Ph.D., Chairperson \\ Dale L. Birkle, Ph.D. \\ Charles R. Craig, Ph.D. \\ Michael I. Luster, Ph.D. \\ Mark J. Reasor, Ph.D. \\ Michael R. Van Scott, Ph.D. \\ Department of Pharmacology and Toxicology \\ Morgantown, West Virginia \\ 2000
}

Keywords: airway, asthma, electrophysiology, epithelium, epithelium-derived relaxing factor, guinea pig, ion channel, ion transporter, lipopolysaccharide, lung, pharmacology, reactivity, respiratory, smooth muscle, tonicity, trachea 


\section{Abstract \\ Characterization of the Airway Epithelial Bioelectric Mechanisms Associated with the Effects of Epithelium-Derived Relaxing Factor in Guinea-Pig Isolated Trachea}

\section{Richard A. Johnston}

In response to elevated serosal or mucosal tonicity, guinea-pig tracheal epithelium releases the non-nitric oxide, non-prostanoid, epithelium-derived relaxing factor (EpDRF), which subsequently diffuses to the underlying airway smooth muscle to initiate relaxation. Hypertonicity-induced smooth muscle relaxation via EpDRF involves epithelial $\mathrm{Na}^{+}$and $\mathrm{Cl}^{\text {! }}$ channels and is preceded by a depolarization of the transepithelial potential difference $\left(\mathrm{V}_{\mathrm{ms}}\right)$. By utilizing two different experimental systems, this study sought to characterize further the airway epithelial bioelectric mechanisms associated with the effects of EpDRF in guinea-pig isolated trachea.

First, this study attempted to elucidate which epithelial ion channels and/or transporters are involved in the depolarization of the $\mathrm{V}_{\mathrm{ms}}$. This was accomplished by determining the effect of ion channel and transporter inhibitors on in vitro transepithelial short-circuit current responses to elevated mucosal tonicity in guinea-pig tracheal segments mounted in Ussing chambers. The data from these studies indicate that apical $\mathrm{Na}^{+}$channels and the basolateral $\mathrm{Na}^{+}-\mathrm{K}^{+}-2 \mathrm{Cl}^{\text {! }}$ cotransporter are intimately involved in the epithelial bioelectric response to elevated mucosal tonicity, and thus, may play an important role in the synthesis, release, and/or effects of EpDRF.

Secondly, this study sought to determine the consequences of altered epithelial ion transport, following LPS-treatment (4 mg/kg, i.p.; $18 \mathrm{hr}$ post-treatment), on EpDRF-induced smooth muscle relaxation and its associated bioelectric events as well as airway reactivity to methacholine (MCh). In vitro tracheal smooth muscle contractile and relaxant responses as well as $\mathrm{V}_{\mathrm{ms}}$ responses were measured utilizing the isolated and modified isolated, perfused trachea apparatuses while the two-chambered, whole-body plethysmograph was used to measure in vivo airway reactivity. LPS-treatment caused numerous and complex changes in EpDRF-induced smooth muscle relaxation and its associated bioelectric events. In addition, LPS-treatment causes in vivo airway hyporeactivity to $\mathrm{MCh}$. These results suggest that the effects of EpDRF on smooth muscle reactivity are modulated by changes in the electrical activity of the epithelium which can ultimately impact on airway reactivity to $\mathrm{MCh}$. 


\section{Table of Contents}

Characterization of the Airway Epithelial Bioelectric Mechanisms Associated with the Effects of Epithelilm-Derived Kelaxing Hactor in Gillinea-Pig Isolafed Crachea_ I Ahstract

Table of Contents

List of Tables

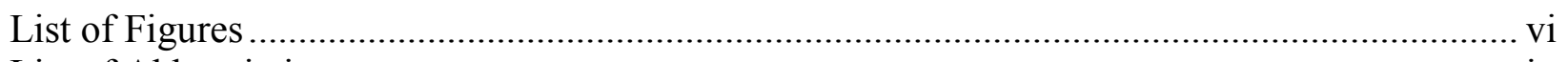

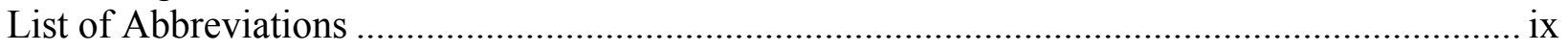

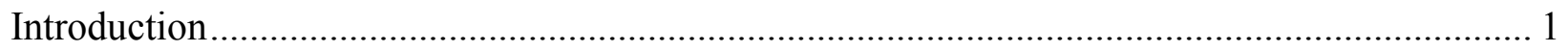

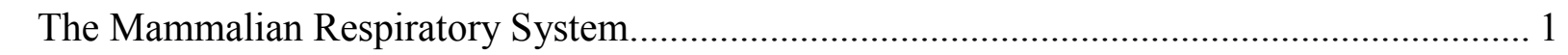

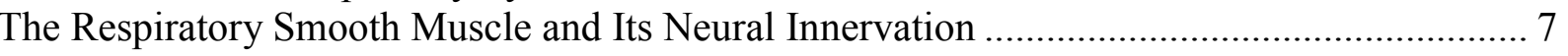

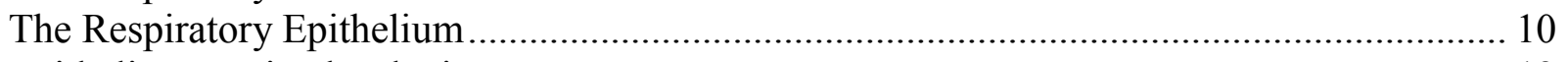

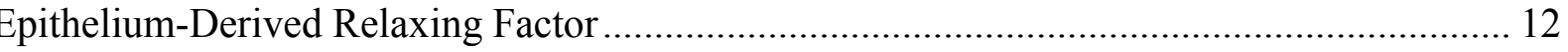

Eell- - olume Regulation in Response to Hypertonic Challenge $\ldots \ldots \ldots \ldots \ldots \ldots \ldots \ldots \ldots \ldots \ldots \ldots \ldots \ldots .18$

xercise-Induced Asthma_ _

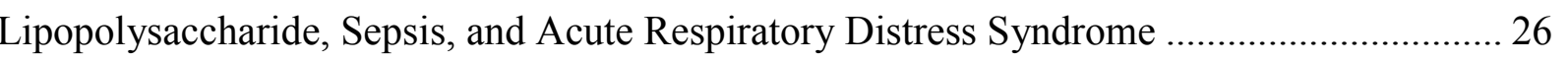

The Relationshin Between Enithelial Bioelectric Resnonses and Enithelium-Derived Relaxing

Factor-Induced Airway Smooth Muscle Relaxation in Response to Hypertonic Solution .......... 30

Dbjectives ................................................................................................. 31

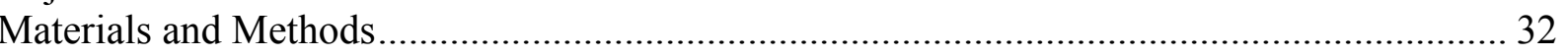

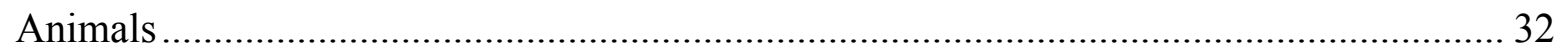

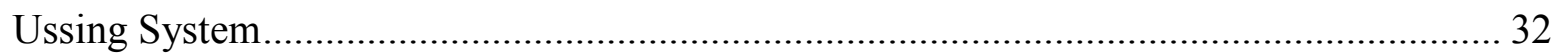

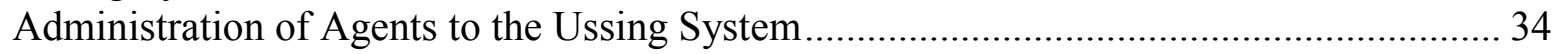

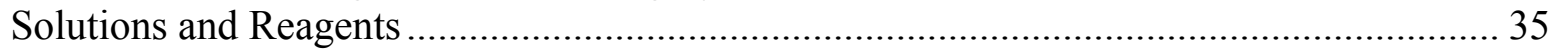

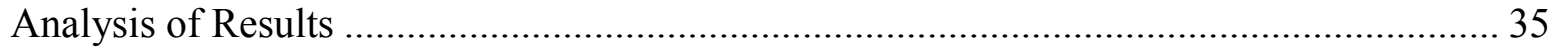

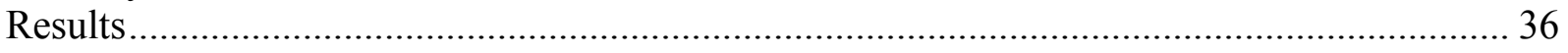

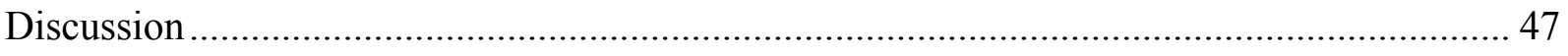

Lipopolvsaccharide-Induced Alferations in_EpitheliaL_Electrophysiology in Kelation to

Epithelium-Derived Relaxing Factor and Airwav Reactivity 55

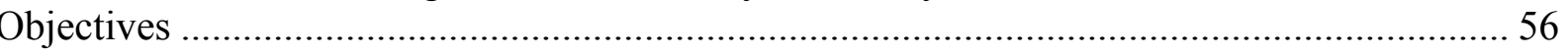

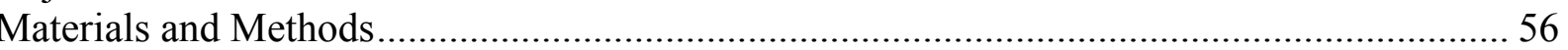

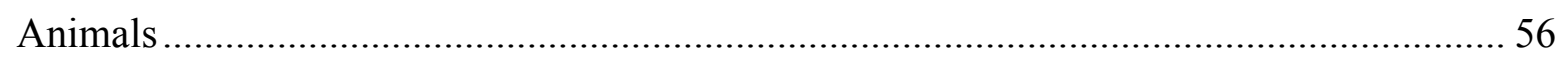

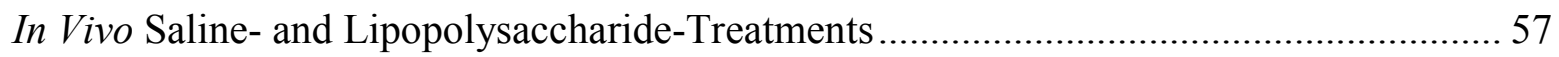

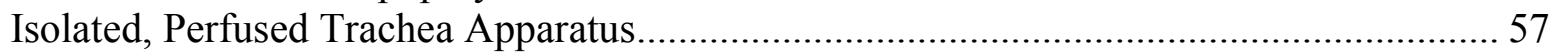

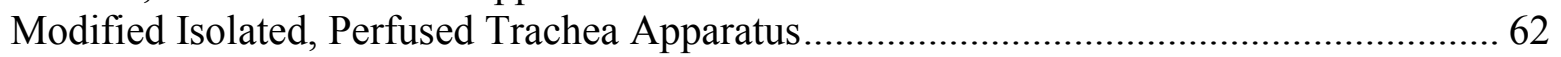

In Vitro Elevated Tonicity Concentration-Response Curves ...................................... 64

In Vitro Effects of Agents on EpDRF-Induced Smooth Muscle Relaxation ...................... 64

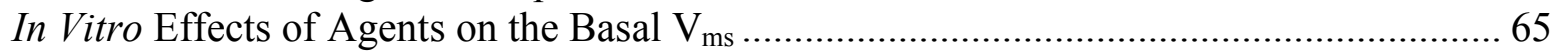

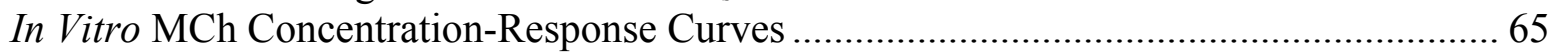

Pracheal thithelilim kemoval os

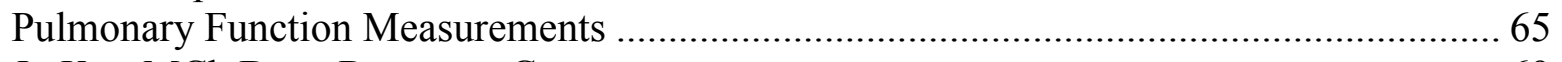

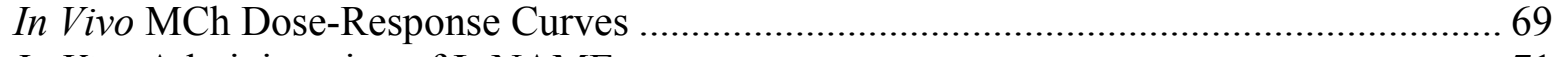

In Vivo Administration of L-NAME....................................................................... 71 


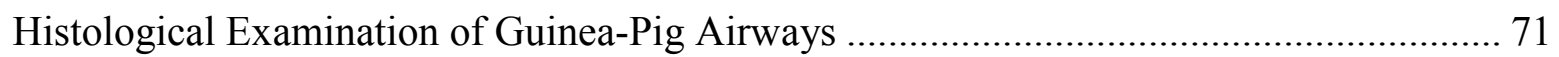

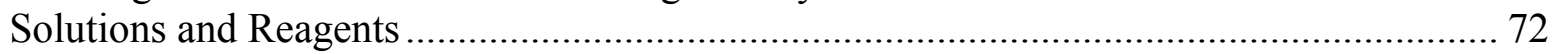

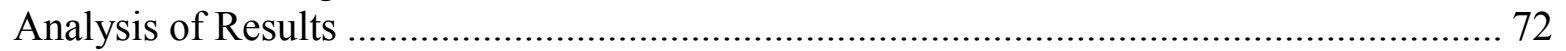

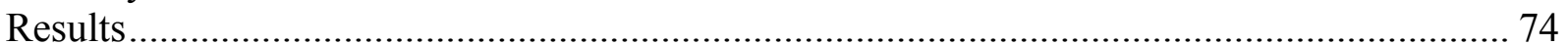

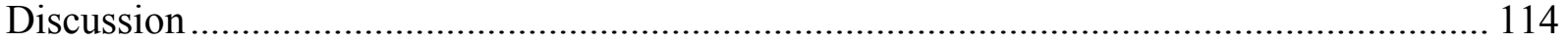

\begin{tabular}{lll}
\hline Conclusions & \\
\hline
\end{tabular}

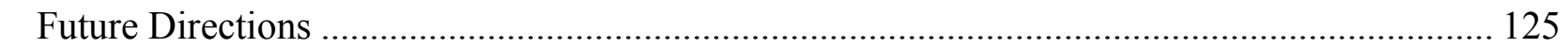

Diterature Cited 127 


\section{List of Tables}

1 Effect of LPS-treatment on in vitro airway reactivity to EpDRF in isolated, perfused trachea

2 Relationship between in vitro smooth muscle relaxation and epithelial depolarization in

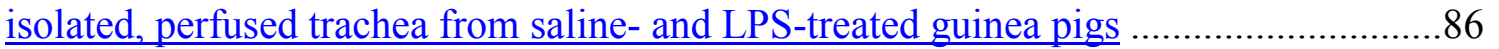

3 Effect of LPS-treatment on in vitro epithelial bioelectric reactivity to hypertonic $\mathrm{NaCl}$ in

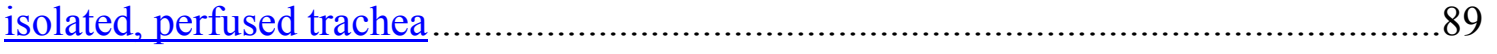

4 Effect of LPS-treatment on in vitro airwav reactivity to $\mathrm{MCh}$ in enithelium-intact.

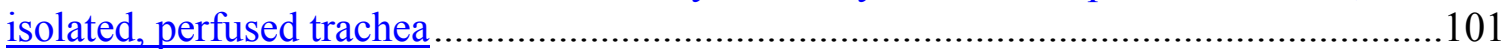

5 Effect of LPS-treatment on in vitro airway reactivity to MCh in epithelium-denuded, isolated, perfused trachea .................................................................................... 103

6 Effect of LPS-treatment on in vitro epithelial bioelectric reactivity to MCh in isolated,

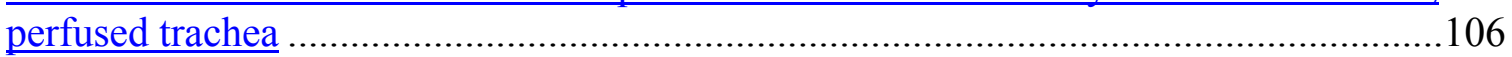

7 Effect of saline- and LPS-treatments on in vivo airway reactivity to inhaled MCh. .......109 


\section{List of Figures}

Divisions of the tracheobronchial tree

Epithelial cell morphology of the proximal and distal airways of the conducting zone. 5

The air-blood barrier

Mechanisms of airway smooth muscle contraction and relaxation ...............................9

Airway surface liquid. .............................................................................. 11

Distribution of airway epithelial ion channels and transporters and the major ion transport

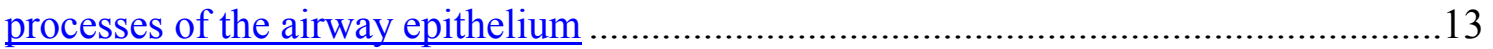

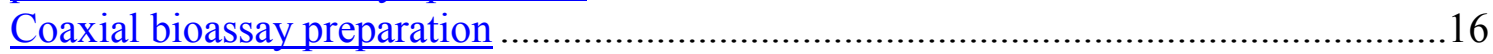

8

Actions of the airway epithelium-derived relaxing factor ...............................................2

Vechanisms of reouluarory volume increase

The elfects of airway drying on the alirway enithelium and airwav wall .......................2.

Localization of linonolvsaccharide and its chemical comnonents ..............................22

hypertonic with added $\mathrm{NaCl}$ or D-mannitol on $I_{\mathrm{sc}}$ in the presence of serosally-applied

MCh in isolated trachea.

Effect of mucosally-applied amiloride on the $I_{\text {sc }}$ responses of mucosally-applied MKH solution made hypertonic with added $\mathrm{NaCl}$ or D-mannitol in the presence of serosallyapplied MCh in isolated trachea .........................................................................39

15 Effect of serosally-applied bumetanide on the $I_{\text {sc }}$ responses of mucosally-applied MKH solution made hypertonic with added NaCl or D-mannitol in the presence of serosallyanded vich in isolated frachea mucosallv-applied MKH solution made hvpertonic with added $\mathrm{NaCl}$ or D-mannitol in the

bresence of serosallv-anolied MCh in isolated trachea

Effect of mucosally-applied NPPB on the $I_{\mathrm{sc}}$ responses of mucosally-applied MKH solution made hypertonic with added $\mathrm{NaCl}$ or D-mannitol in the presence of serosally-

applied MCh in isolated trachea

Effect of serosally-applied ouabain on the $I_{\text {sc }}$ responses of mucosally-applied MKH solution made hypertonic with added $\mathrm{NaCl}$ or D-mannitol in the presence of serosally-

applied MCh in isolated trachea ...........................................................................43

Lack of effect of mucosally- and serosally-applied DMSO on the $I_{\text {sc }}$ responses of

mucosally-applied MKH solution made hypertonic with added NaCl or D-mannito in the

presence of serosally-apphed $\mathrm{VI}$ ch in isolafed irachea _ 45

Comparison of the Iirs and second ce responses io mucosally-applied MKH solution

made hvnertonic with added $\mathrm{NaCl}$ or D-mannitol in the presence of serosally-annlied

MCh in isolated trachea .46

Isolated. perfused trachea apparatus ……..................................................................59

22 Differential pressure measurement in the isolated, perfused trachea apparatus ...............61

$23 \quad \mathrm{~V}_{\mathrm{ms}}$ measurement in the modified isolated, perfused trachea apparatus. .........................63

$24 \quad$ Two-chambered, whole-body plethysmograph. .........................................................67

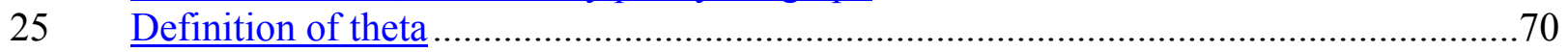

26 Effect of LPS-treatment on the basal $\mathrm{V}_{\text {ms }}$ in isolated, perfused trachea ...........................75 
27 Effect of LPS-treatment on EpDRF-induced smooth muscle relaxation elicited with IL

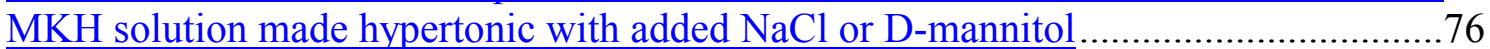

28 Effect of LPS-treatment on EpDRF-induced smooth muscle relaxation elicited with IL MKH solution made hypertonic with added urea

29 Lack of effect of LPS-treatment on EpDRF-1nduced smooth muscle relaxation elicited

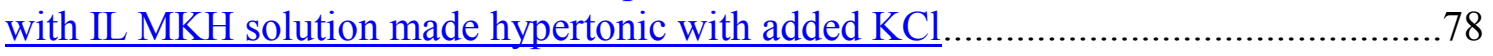

30 ack of effect of exfralliminallv-and infralliminallv-annled indomethacin on En DREmediated relaxation responses elicited with IL MKH solution made hvpertonic with added $\mathrm{NaCl}$ Lack of effect of extraluminally- and intraluminallv-applied L-NAME on EpDRFmediated relaxation responses elicited with IL MKH solution made hypertonic with

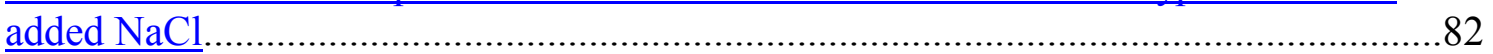

32 Effect of LPS-treatment on epithelial bioelectric reactivity to hypertonic $\mathrm{NaCl}$ in the presence of extraluminally-added MCh ........................................................... 84

33 Effect of LPS-treatment on the relationship between EpDRF-induced smooth muscle

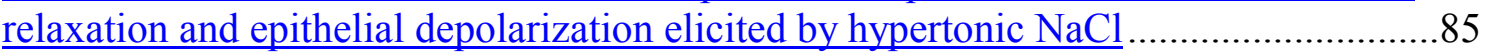

34 Effect of LPS-treatment on epithelial bioelectric reactivity to extraluminally- and infraliminaly-applied NaCl in the absence of MICh (a within-treatment group comparison t 87

Effect of LPS-treatment on enithelial bioelectric reactivity to extraluminally- and intraluminally-applied $\mathrm{NaCl}$ in the absence of $\mathrm{MCh}$ (a between-treatment group comparison). Effect of indomethacin on the basal $\mathrm{V}_{\mathrm{ms}}$ of isolated, perfused trachea from saline-and

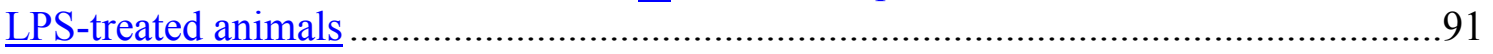

37 Effect of L-NAME on the basal $\mathrm{V}_{\mathrm{ms}}$ of isolated, perfused trachea from saline and LPS-

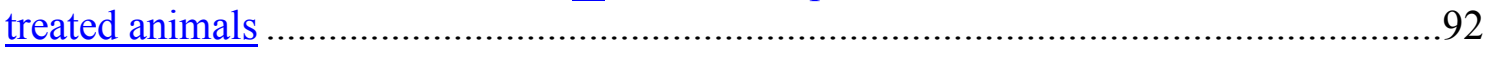

38 Effect of atropine on the basal $\mathrm{V}_{\mathrm{ms}}$ of isolated, perfused trachea from saline and LPS-

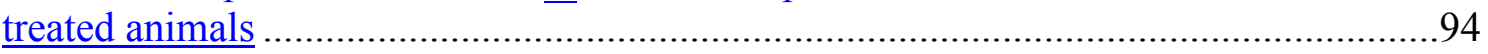

39 Effect of SNP on the basal $\mathrm{V}_{\text {ms }}$ of 1solated, perfused trachea from saline- and LPS treated animals os

40 Effect of amiloride on the hasal V of isolated perfused trachea from saline and LPStreated animals 96

41 Effect of the in vitro addition of LPS on the basal $V_{\text {ma }}$ of isolated nerfused trachea from

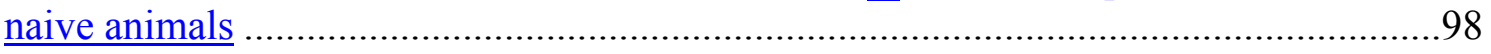

42 Lack of effect of LPS-treatment on tracheal smooth muscle reactivity to extraluminallyand intraluminally-applied MCh in epithelium-intact and denuded isolated, perfused

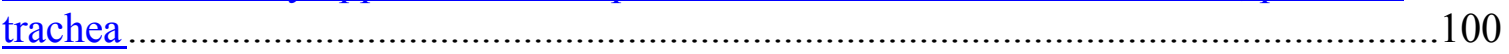

43 Effect of LPS-treatment on epithelial bioelectric reactivity to extraluminally- and

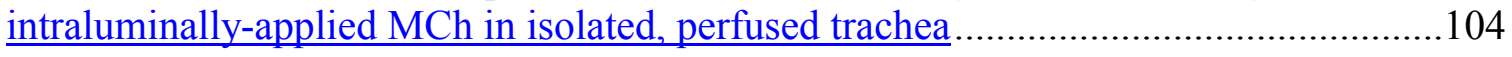

44 Effect of saline- and LPS-treatments on pre- and post-treatment basal specific airway

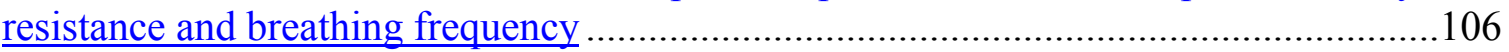

45 Effect git saline- and LPS-treatments on $i n$ vivo airway reactivity to inhaled MCh 107

46 Lack of effect of L-NAME on in vive airway reactivity to inhaled MCh aerosol in salineand LPS-treated guinea pigs. ............................................................................... 110 
47 Histological sections of guinea-pig airways following saline- and

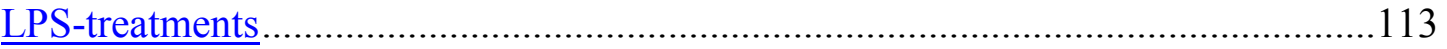




\section{List of Abbreviations}

$\mathrm{ACh}$

ARDS

ASL

ASM

$\mathrm{BF}$

C.I.

DMSO

$\mathrm{EC}_{50}$

EIA

EL

EpDRF

IL

$I_{\mathrm{sc}}$

$\mathrm{KCl}$

L-NAME

LPS

$\mathrm{MCh}$

MCh $\mathrm{PC}_{200}$

$\mathrm{MKH}$

$\mathrm{NaCl}$

NO

RVI

SRaw

$\mathrm{V}_{\mathrm{ms}}$

$\triangle \mathrm{P}$ acetylcholine

acute respiratory distress syndrome

airway surface liquid

airway smooth muscle

breathing frequency

confidence interval

dimethyl sulfoxide

effective concentration producing $50 \%$ of the maximum response

exercise-induced asthma

extraluminal

epithelium-derived relaxing factor

intraluminal

short-circuit current

potassium chloride

$\mathrm{N}^{\top}$-nitro-L-arginine methyl ester

lipopolysaccharide

methacholine chloride

provocative methacholine chloride concentration producing a twofold increase in basal specific airway resistance

modified Krebs-Henseleit

sodium chloride

nitric oxide

regulatory volume increase

specific airway resistance

transepithelial potential difference

differential pressure (inlet minus outlet pressure difference) 


\section{Introduction}

\section{The Mammalian Respiratory System}

The mammalian respiratory system is comprised of the lungs, the extra- and intrapulmonary airways, the chest wall, and the respiratory control centers of the central nervous system (Levitzky, 1995). The primary function of the respiratory system is to exchange gases between the atmosphere and the blood. This ensures that mammals are supplied with oxygen while carbon dioxide is removed (West, 1995; Johnson, 1997). Oxygen, in conjunction with other organic molecules, is utilized by the cell to produce energy in the form of adenosine triphosphate during the process of oxidative phosphorylation (Lehninger et al., 1993). Aside from gas exchange, the respiratory system has non-respiratory functions which include playing a significant role in (1) acid-base balance, (2) body defense, (3) metabolism, (4) olfaction, (5) phonation, and (6) water loss (Levitzky, 1995).

In order for cells to be supplied with oxygen, the oxygen within the inspired air must first gain access to the alveoli, or gas exchanging regions of the lung, before being distributed throughout the body by means of the circulatory system. Air is primarily inspired through the nose and to a lesser extent, the mouth, and once inspired, it passes through the pharynx, larynx, trachea, bronchi, and bronchioles until it eventually reaches the alveoli. The most distal portion of the trachea branches into right and left primary bronchi which enter the right and left lungs, respectively. The right and left primary bronchi continue to branch even further into at least two smaller daughter branches. The airways continue to branch until terminating into alveolar sacs (Weibel, 1963; Nagaishi et al., 1972; Curtis and Barnes, 1989; Sherwood, 1997). Figure 1 illustrates the branching of the tracheobronchial tree, and because of anatomical and functional differences along its length, it is divided into a conducting zone and a respiratory or transitional zone. The conducting zone consists of airway generations zero to sixteen while generations seventeen to twenty-three make up the respiratory or transitional zone (Weibel, 1963). 
Gas exchange is restricted to the respiratory or transitional zone while the conducting zone constitutes an anatomic dead space (Levitzky, 1995). When air moves through the conducting airways, it is humidified and warmed to body temperature (Levitzky, 1995; Sherwood, 1997). The respiratory or transitional zone occupies the last $5 \mathrm{~mm}$ of each airway branch and consists of small airways, namely bronchioles and alveoli, which are capable of gas exchange (Bouhuys, 1977). In addition to the functional differences between the conducting and respiratory or transitional zones, there is also a great deal of structural heterogeneity in the airways within and between these two regions.

The airways of the upper conducting zone are relatively nonmuscular, rigid tubes encircled by cartilaginous rings. Their walls are rich in connective tissue and cartilage but sparsely populated with smooth muscle (Johnson, 1997). The luminal surface of these airways are predominately lined with ciliated, pseduostratified, columnar epithelial cells, among which are dispersed submucosal glands, basal, brush, goblet, Kulchitsky, and serous cells as well as a small number of neuroendocrine cells

(Divertie and Brass, 1980; Fawcett and Jensh, 1997; Johnson, 1997). 


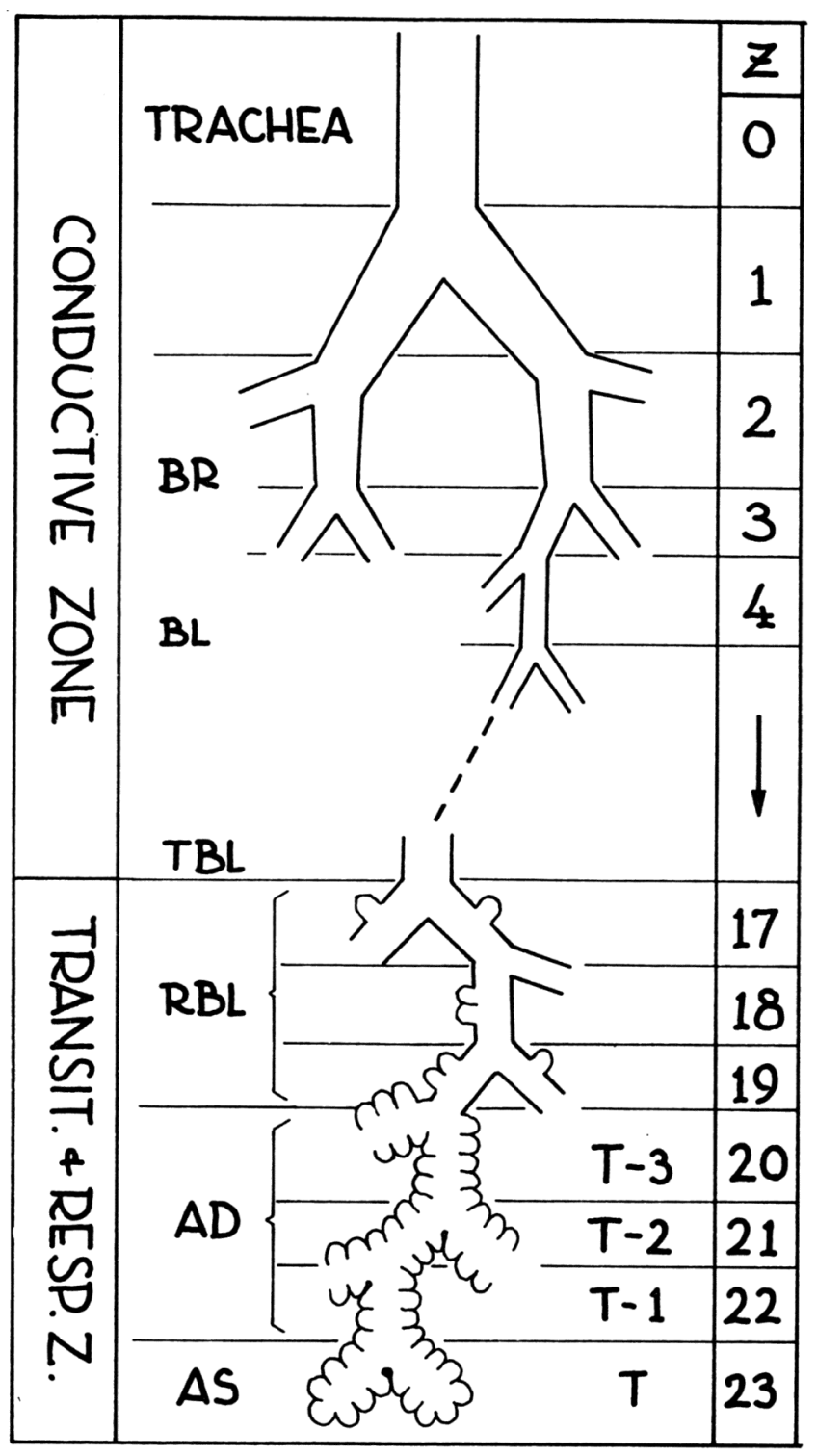

Figure 1. Divisions of the tracheobronchial tree. Z, generation; BR, bronchus; BL, bronchiole; TBL, terminal bronchiole; RBL, respiratory bronchiole; AD, alveolar duct; AS, alveolar sac. Reprinted with permission from Weibel ER (1963) Morphometry of the Human Lung, Academic Press Ltd, London UK. 
The airways located at the junction between the conducting and respiratory or transitional zones differ from those either proximal or distal to them. Their walls are devoid of cartilage and contain little connective tissue but are rich in smooth muscle (Weibel, 1963; Nagaishi et al., 1972; Johnson, 1997). Ciliated, cuboidal epithelial cells are the predominant epithelial cell type of this region; however, they are smaller than their more proximal counterparts. There are fewer goblet cells in this region while submucosal glands are virtually absent. Clara cells, secretory cells of ambiguous function, also populate this region (Divertie and Brass, 1980; Fawcett and Jensh, 1997; Johnson, 1997). The differences in epithelial cell morphology within the conducting zone are shown in Figure 2.

The airway walls of the respiratory or transitional zone lack smooth muscle and contain very little connective tissue (Johnson, 1997). Type I alveolar epithelial cells are the predominant cell type covering the luminal surface of the alveoli. They are long, thin cells lacking cilia. Type II alveolar epithelial cells, which synthesize and secrete surfactant, are also found, to a lesser extent, along the luminal surface of the alveoli (Sherwood, 1997). In order for gas exchange to occur between the alveoli and the blood in the pulmonary capillaries, the gas must diffuse through the air-blood barrier which is formed by the pulmonary capillary endothelium, the type I alveolar epithelium, and the interstitial space between the two (Sherwood, 1997). An illustration of the air-blood barrier is shown in Figure 3. There are an estimated 300 million alveoli and nearly 1000 pulmonary capillaries surrounding each alveolus, providing a 50-100 $\mathrm{m}^{2}$ surface area for gas exchange (Divertie and Brass, 1980; West, 1995). When this is taken with the fact that the air-blood barrier is only $0.5 \mu \mathrm{m}$ thick, the mammalian respiratory system is indeed an exquisitely efficient gas exchanger (West, 1995; Sherwood, 1997). 

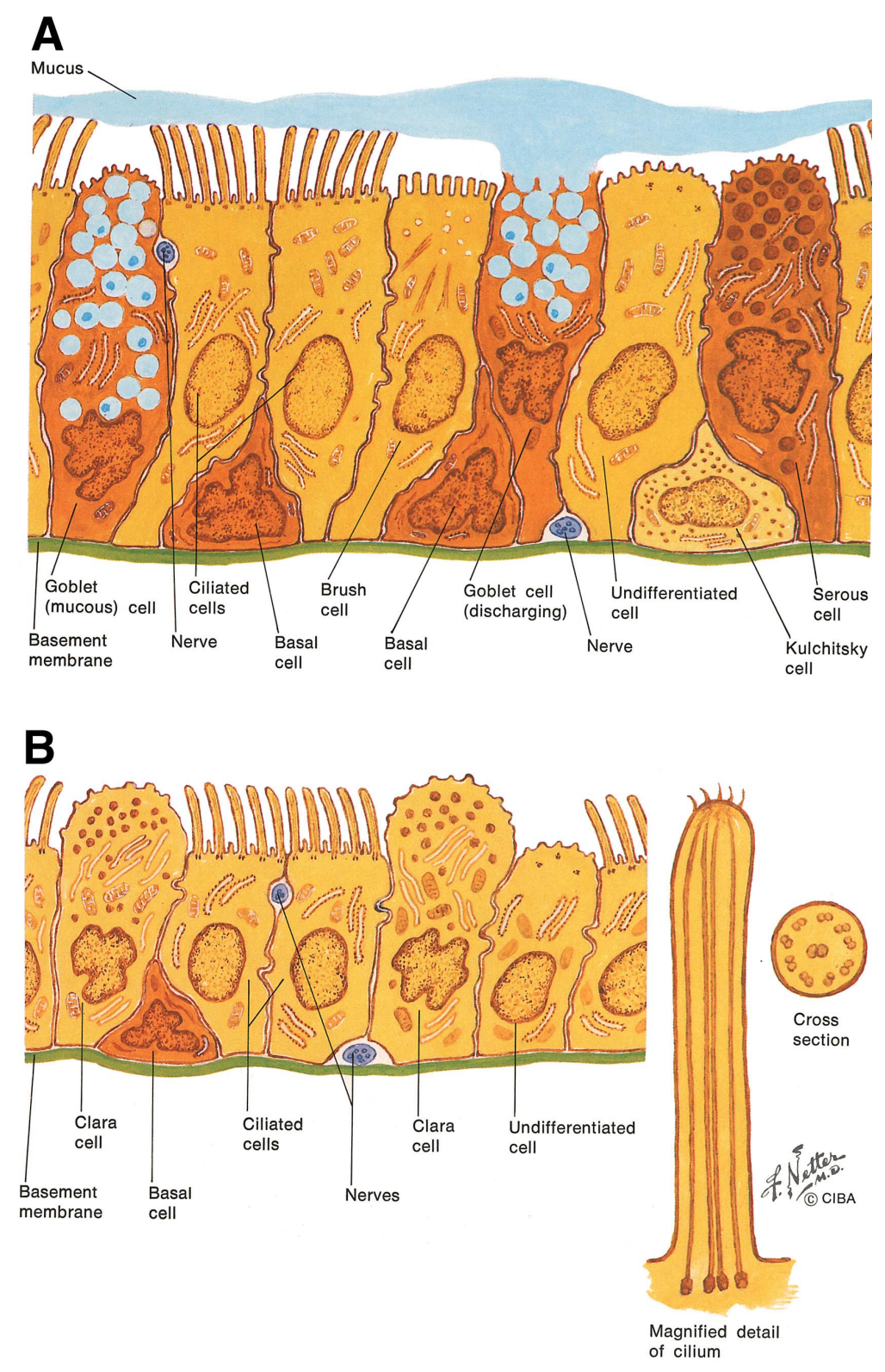

Figure 2. Epithelial cell morphology of the (A) proximal and (B) distal airways of the conducting zone. Along the respiratory tract, there are distinct differences in epithelial cell morphology. Copyright 1980. Novartis. Reprinted with permission from Novartis Medical Education, illustrated by Frank H. Netter, MD. All rights reserved. 


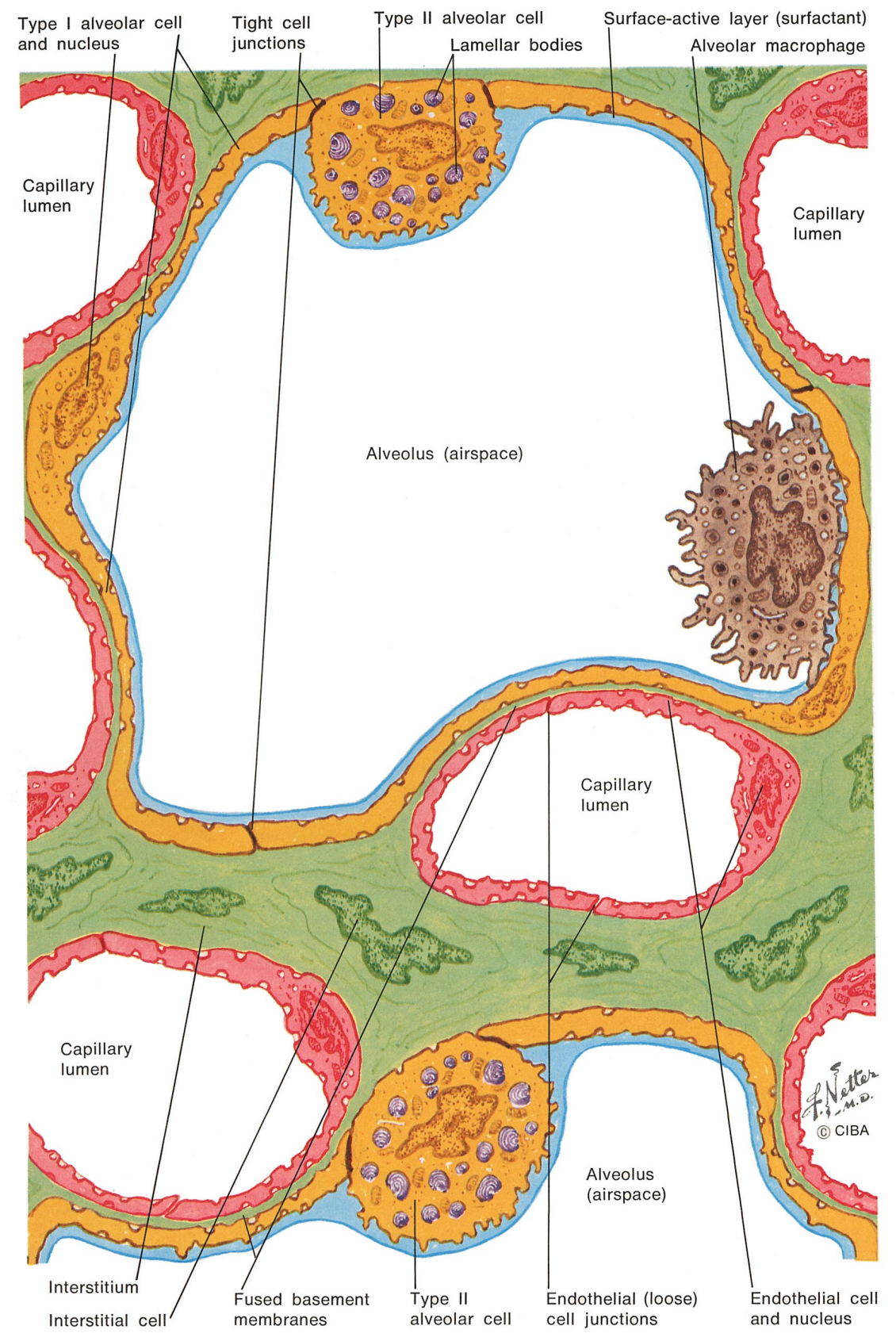

Figure 3. The air-blood barrier. This illustration depicts two of an estimated 300 million alveoli, the gas exchanging regions of the lung, and the other, affiliated structures of the airblood barrier. Copyright 1980. Novartis. Reprinted with permission from Novartis Medical Education, illustrated by Frank H. Netter, MD. All rights reserved. 


\section{The Respiratory Smooth Muscle and Its Neural Innervation}

The respiratory or airway smooth muscle is one of the six basic types of smooth muscle (Horowitz et al., 1996). It has many structural features in common with the other types of smooth muscle and is found from the trachea to the respiratory bronchioles, albeit in different quantities.

The parasympathetic nervous system has a profound impact on airway smooth muscle contraction. Postganglionic parasympathetic fibers directly innervate the smooth muscle. The release of acetylcholine (ACh) from these neurons initiates smooth muscle contraction via smooth muscle $\mathrm{M}_{3}$ cholinoceptors which are coupled to $\mathrm{G}_{\mathrm{q}}$-proteins on the muscle cells. Other spasmogens, by binding to their appropriate receptors, also initiate smooth muscle contraction through the activation of $\mathrm{G}_{\mathrm{q}}$-proteins. The " ${ }_{\mathrm{q}}$ subunit of the $\mathrm{G}_{\mathrm{q}}$-protein activates the inner, plasma membrane-bound enzyme phospholipase $\mathrm{C}$-\$, leading to the synthesis of inositol 1,4,5trisphosphate $\left(\mathrm{IP}_{3}\right)$ and diacylglycerol from a membrane-bound phospholipid, phosphatidylinositol 4,5 bisphosphate. $\mathrm{IP}_{3}$ elevates cytosolic $\mathrm{Ca}^{2+}$ levels by stimulating, either directly or indirectly, the release of $\mathrm{Ca}^{2+}$ from intracellular stores (sarcoplasmic reticulum and mitochondria), the influx of extracellular $\mathrm{Ca}^{2+}$ through $\mathrm{Ca}^{2+}$-selective ion channels and/or transporters, and $\mathrm{Ca}^{2+}$-induced $\mathrm{Ca}^{2+}$ release. Elevations in cytosolic $\mathrm{Ca}^{2+}$ activate the $\mathrm{Ca}^{2+} /$ calmodulin-dependent myosin light chain kinase which enhances actin-myosin interactions resulting in smooth muscle contraction (Figure 4A) (Gross, 1988; Allen and Walsh, 1994; Horowitz et al., 1996; Janssen et al., 1998).

The sympathetic nervous system can also influence airway smooth muscle tone. There is evidence that postganglionic sympathetic fibers terminate on postganglionic parasympathetic nerves, thereby having the capability to modulate cholinergic neural mechanisms (Ind and Barnes, 1988). Activation of smooth muscle $\$ 2$-adrenoceptors by adrenergic agonists initiates bronchodilation by activating adenylate cyclase which increases intracellular cyclic adenosine monophosphate (cAMP) levels. cAMP stimulates smooth muscle relaxation by down- 
regulating myosin light chain kinase activity, stimulating the uptake of $\mathrm{Ca}^{2+}$ into the sarcoplasmic reticulum, and activating $\mathrm{Ca}^{2+}$-dependent $\mathrm{K}^{+}$channels (Figure 4B) (Kotlikoff and Kamm, 1996; Janssen et al., 1998). In humans, prostaglandin $\mathrm{E}_{2}$ is capable of elevating intracellular smooth muscle cAMP concentrations and initiating relaxation. " Adrenoceptors are also present on airway smooth muscle, but the consequences of their activation are more controversial (Ind and Barnes, 1988).

Besides the autonomic nervous system, there are other neural systems capable of modulating airway smooth muscle tone. The inhibitory nonadrenergic, noncholinergic (iNANC) system is the only significant bronchodilator pathway in humans. Vasoactive intestinal peptide (VIP) and nitric oxide (NO) are the major neurotransmitters of this system (Barnes, 1988). VIP exerts its bronchodilating effects by elevating intracellular cAMP levels while NO initiates bronchodilation by activating soluble guanylate cyclase, resulting in the activation of smooth muscle $\mathrm{K}^{+}$channels via cyclic guanosine monophosphate (cGMP) (Figure 4B) (Janssen et al., 1998). The excitatory nonadrenergic, noncholinergic (e-NANC) system is another neural system capable of influencing airway smooth muscle tone. e-NANC fibers release substance $\mathrm{P}$ and tachykinins from their terminals located beneath the airway epithelium. Both substance $\mathrm{P}$ and tachykinins initiate smooth muscle contraction by activation of their respective $\mathrm{G}_{\mathrm{q}}$-protein-coupled receptors (Barnes, 1988; Janssen et al., 1998). 

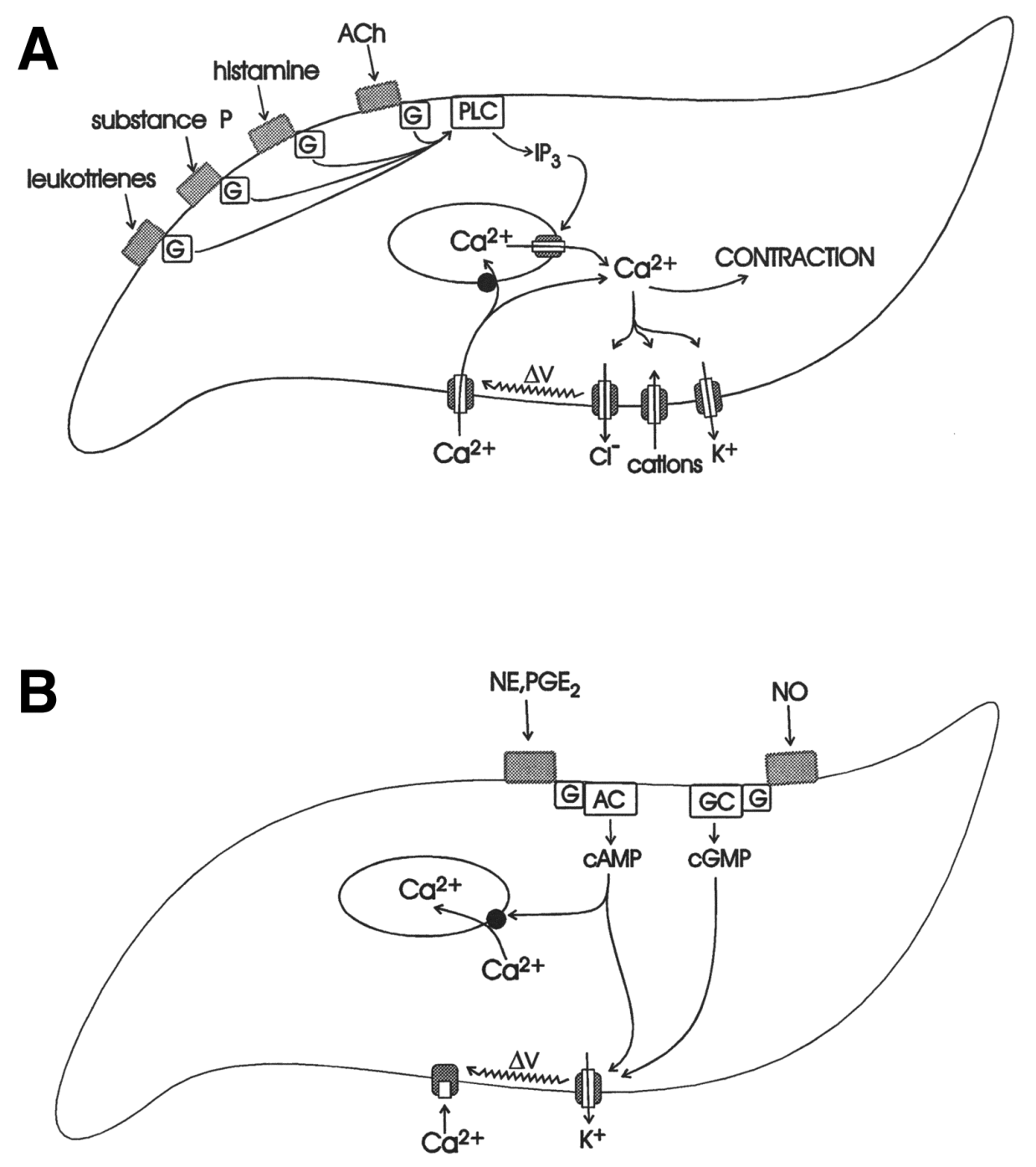

Figure 4. Mechanisms of airway smooth muscle (A) contraction and (B) relaxation. See text for details. ACh, acetylcholine; G, G-protein-coupled receptor; PLC, phospholipase C-\$; $\mathrm{IP}_{3}$, inositol 1,4,5-trisphosphate; ) $\mathrm{V}$, change in membrane potential: (A) depolarization and (B) hyperpolarization; $\mathrm{NE}$, norepinephrine; $\mathrm{PGE}_{2}$, prostaglandin $\mathrm{E}_{2}$; $\mathrm{NO}$, nitric oxide; $\mathrm{AC}$, adenylate cyclase; GC, soluble guanylate cyclase; cAMP, cyclic adenosine monophosphate; cGMP, cyclic guanosine monophosphate. Reprinted with permission from Janssen LJ, Daniel EE and Rodger IW (1998) Airway smooth muscle cells: Structure and function, in Asthma: Basic Mechanisms and Clinical Management (Barnes PJ, Rodger IW and Thomson NC eds) pp 90-112, Academic Press Ltd, London UK. 


\section{The Respiratory Epithelium}

The respiratory or airway epithelium constitutes a barrier to control the movement of substances between the external and internal environments of the body (Hulsmann and Jongste, 1996; Fawcett and Jensh, 1997); however, the epithelium functions as more than just a simple diffusion barrier (Widdicombe, 1991; Folkerts and Nijkamp, 1998). Under normal physiological conditions, the respiratory epithelium also functions as a mucus transporter (Widdicombe, 1991; Clarke and Boucher, 1993), a regulator of ion and fluid transport (Clarke and Boucher, 1993; Boucher, 1999), an osmotic sensor

(Willumsen et al., 1994; Dortch-Carnes et al., 1999; Fedan et al., 1999), a site of metabolism for both endogenous and exogenous substances, and a source of spasmogenic and relaxant substances which are capable of modulating the reactivity of the underlying airway smooth muscle

(Flavahan et al., 1985; Fedan et al., 1988; Goldie and Hay, 1997; Folkerts and Nijkamp, 1998).

Overlying the luminal or mucosal surface of the respiratory epithelium is the airway surface liquid (ASL) which consists of two layers: the sol and the gel. The sol layer is a thin, 5 to $7 \mu \mathrm{m}$ in depth, watery layer which blankets the surface. Its thickness approximates the length of the cilia extending from the luminal surface of the epithelium. The gel layer rests upon the sol layer and is a discontinuous mucus blanket, consisting of $95 \%$ water (Figure 5). Since the mucus is both elastic and viscous, it can easily trap, and subsequently hold, inhaled, foreign matter. As the cilia beats, both the sol and gel layers move in tandem in a cephalad direction which allows for the effective clearance of inhaled, foreign substances and microorganisms from the respiratory tract. Epithelial ion and fluid transport play a major role in determining the depth of the ASL (Clarke and Boucher, 1993; Widdicombe and Widdicombe, 1995; Boucher, 1999). 


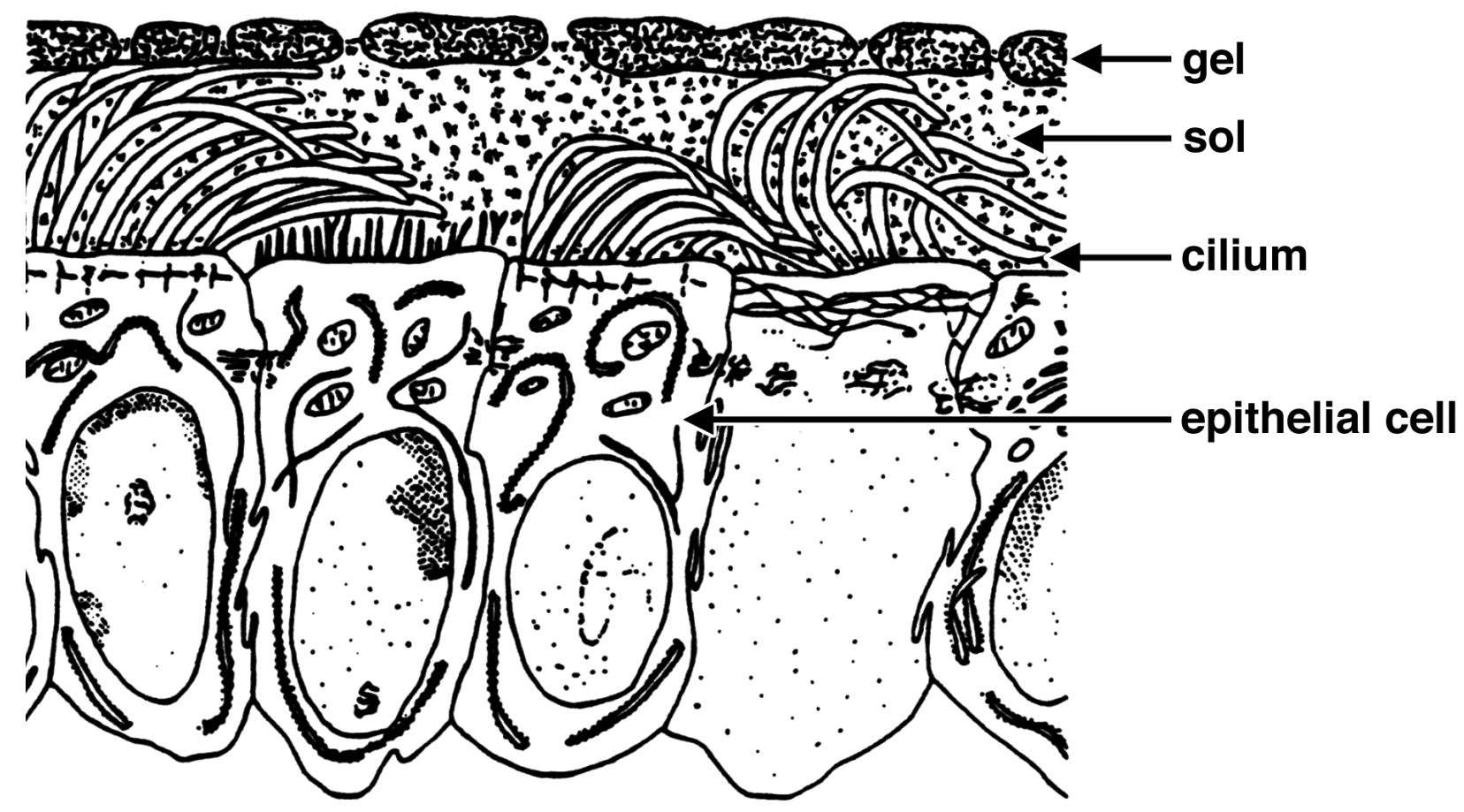

Figure 5. Airway surface liquid (ASL). The ASL blankets the mucosal surface of the airway epithelium and consists of two layers: the sol and the gel. The depth of the ASL ranges between 5-100 $\mu \mathrm{m}$ (Widdicombe and Widdicombe, 1995). Reprinted from Clarke LL and Boucher RC (1993) Ion and water transport across airway epithelia, in Pharmacology of the Respiratory Tract: Experimental and Clinical Research (Chung KF and Barnes PJ eds) pp 505-550 by courtesy of Marcel Dekker Inc. For clarity, the author of this dissertation added the figure labels. 
Under normal, physiological conditions, the epithelium of the conducting airways of most mammalian species, excluding the cow and the dog, is $\mathrm{Na}^{+}$-absorbing (Boucher et al., 1982; Langridge-Smith et al., 1984); however, all generate a lumen-negative potential with respect to the basolateral surface. A tentative localization of airway epithelial ion channels and transporters is shown in Figure 6. The activity of the $\mathrm{Na}^{+}-\mathrm{K}^{+}$-ATPase, localized to the basolateral surface of the epithelial cell, establishes an electrochemical gradient which draws $\mathrm{Na}^{+}$into the cell from the ASL through amiloride-sensitive $\mathrm{Na}^{+}$channels. The $\mathrm{Na}^{+}$is then extruded from the cell across the basolateral membrane by means of the $\mathrm{Na}^{+}-\mathrm{K}^{+}$-ATPase. $\mathrm{K}^{+}$channels and the electroneutral $\mathrm{Na}^{+}-\mathrm{K}^{+}-2 \mathrm{Cl}^{!}$cotransporter also play a role in epithelial ion transport. Under normal, physiological conditions for most mammalian species, there is no net transcellular movement of $\mathrm{Cl}^{!}$; however, in order to achieve electrochemical equilibrium, $\mathrm{Cl}^{!}$moves through the paracellular pathway as $\mathrm{Na}^{+}$is absorbed transcellularly (Figure 6)

(Boucher et al., 1982; Clarke and Boucher, 1993; Knowles et al., 1995).

\section{Epithelium-Derived Relaxing Factor}

The respiratory epithelium can synthesize and release substances which are capable of diffusing to the underlying airway smooth muscle and modulating its tone (Fedan et al., 1988; Morrison et al., 1990; Hulsmann and Jongste, 1996). These autacoids can have either an excitatory or inhibitory effect upon airway smooth muscle tone. The inhibitory, non-nitric oxide, non-prostanoid epithelium-derived relaxing factor (EpDRF) is one such autacoid (Munakata et al., 1988, 1990; Fedan et al., 1999).

In 1985 Flavahan and colleagues provided the first evidence that the respiratory epithelium could modulate the tone of the underlying airway smooth muscle. After mechanical removal of the epithelium from canine bronchial rings, the bronchial smooth muscle became more sensitive to the effects of contractile agonists (ACh, histamine, and 5-hydroxytryptamine). 


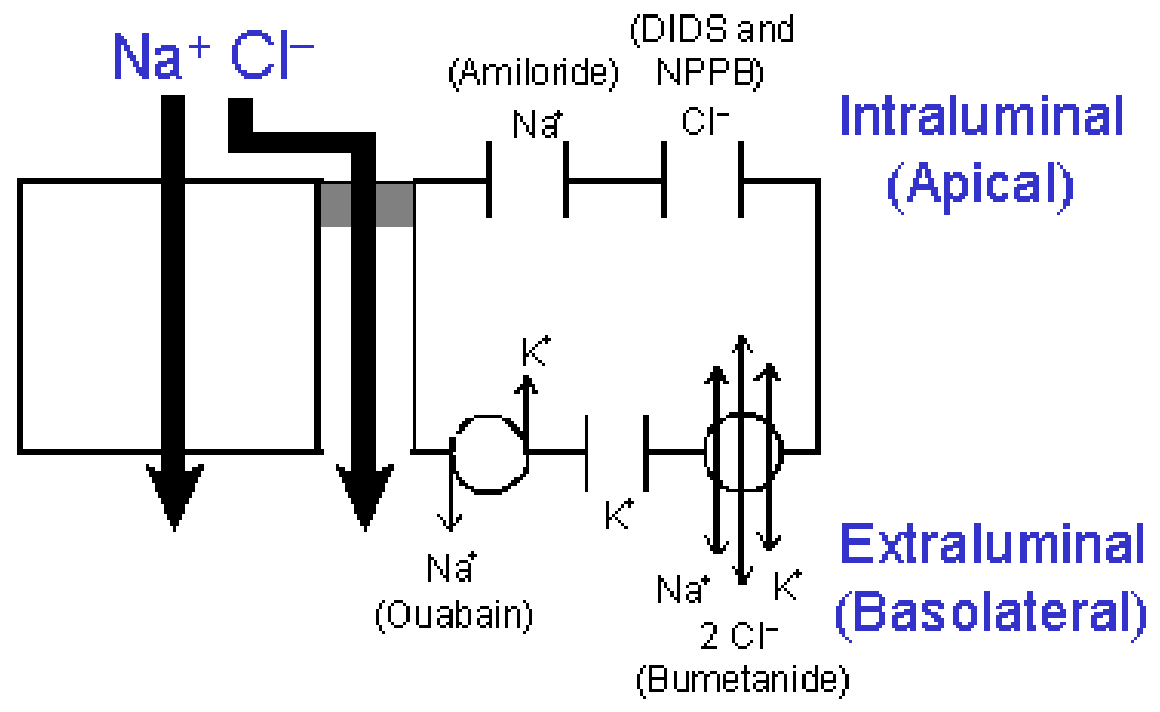

Figure 6. Distribution of airway epithelial ion channels and transporters and the major ion transport processes of the airway epithelium. This illustration holds true for most mammalian species (Boucher et al., 1982; Clarke and Boucher, 1993). The various ion channels and transporters of the airway epithelium are asymmetrically distributed between the apical and basolateral surfaces of these cells. The respective inhibitor of each ion channel and transporter is shown in parentheses. The arrows indicate the net, transcellular movement of $\mathrm{Na}^{+}$and the

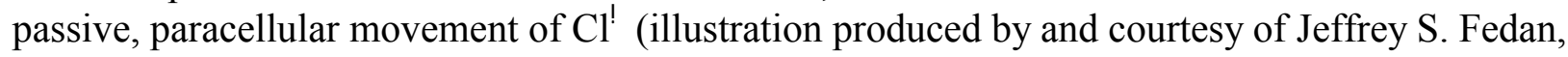
Ph.D.). 
In canine bronchial rings precontracted with $\mathrm{ACh}$, isoproterenol-induced relaxations were attenuated in epithelium-denuded preparations. This same study also demonstrated that contractile responses to transmural nerve stimulation were potentiated by epithelium removal. In epithelium-intact preparations, the peak contraction elicited by a particular electrical frequency was followed by a decrease in tone as the stimulation continued; however, this decrease in tone was absent in epithelium-denuded preparations (Flavahan et al., 1985). Later that same year, a separate group reported that epithelium removal increased the sensitivity of bovine tracheal strips to the contractile effects of $\mathrm{ACh}$, histamine, and 5-hydroxytryptamine (Barnes et al., 1985). In the months and years that followed, other studies with isolated, human (Raeburn et al., 1986a), guinea pig (Hay et al., 1986; Holroyde, 1986; Murlas, 1986), and rabbit (Raeburn et al., 1986b) airway preparations reported that epithelium removal increased the sensitivity of the airway smooth muscle to contractile agonists; however, the effect of epithelium removal upon airway smooth muscle reactivity to relaxant agonists was much more variable. Most of these studies, with the exception of Holroyde (1986) concluded that the increased sensitivity of the smooth muscle to contractile agonists following epithelium removal was due to the loss of an epitheliumderived inhibitory factor, EpDRF, which normally inhibits smooth muscle reactivity to contractile agonists. Some of these same studies also demonstrated that EpDRF was not a metabolite of arachidonic acid (Barnes et al., 1985; Flavahan et al., 1985), but nevertheless, a separate study concluded that the cytochrome $\mathrm{P}_{450}$ enzyme system was involved in epithelial modulation of smooth muscle tone (Raeburn et al., 1988).

The first evidence supporting the hypothesis that the airway epithelium releases an inhibitory factor capable of modulating the reactivity of adjacent smooth muscles came in 1986 with the development of the coaxial bioassay preparation. This preparation consisted of an endothelium-denuded rabbit aortic strip (recipient tissue) positioned within the lumen of an epithelium-intact or -denuded guinea-pig trachea (donor tissue) (Figure 7A). By attaching the endothelium-denuded rabbit aortic strip to an isometric force transducer, contractile and relaxant responses of this tissue could be measured. Following the contraction of the aortic strip with 
phenylephrine, an " ${ }_{1}$-adrenoceptor agonist, ACh was added to the preparation which subsequently relaxed the aortic strip. When the epithelium was removed from the guinea-pig trachea, and the same experiment repeated, the aortic strip failed to relax (Ilhan and Sahin, 1986) (Figure 7B). Coaxial bioassay experiments from other laboratories reported similar results when guinea-pig tracheal smooth muscle or rat anococcygeus muscle were used as the recipient tissue (Güc et al., 1988a; Güc et al., 1988b), or when human bronchus was used as a donor to relax phenylephrine-contracted rat aortic strips (Fernandes et al., 1990). In all of these coaxial bioassay experiments, as in the original, removal of the epithelium from the donor tissue abolished the relaxation response of the recipient tissue, supporting the notion that the relaxation of the recipient tissue was dependent upon an epithelium-derived relaxing factor, EpDRF. Further pharmacological characterization of EpDRF with the coaxial bioassay preparation demonstrated that this factor was not a product of nitric oxide synthase, cyclooxygenase, lipoxygenase, or the cytochrome $\mathrm{P}_{450}$ enzyme systems. This same study showed that EpDRF was not related to free radical production, soluble guanylate cyclase, \$-adrenoceptors, or platelet-activating factor (Fernandes and Goldie, 1990).

Until the late 1980s, EpDRF was studied using rings or strips of airway tissue, which had limited usefulness. In 1988 the guinea-pig isolated, perfused trachea apparatus was developed, allowing for much more elegant EpDRF studies (Munakata et al., 1988). This preparation allowed pharmacological agents to be added separately to either the extraluminal (serosal) or intraluminal (mucosal) surfaces of the trachea. In this preparation, contractile agonists added to the extraluminal surface were more potent since they had direct access to the smooth muscle. Agents added to the intraluminal surface must first diffuse through the epithelium before interacting with the smooth muscle. Early experiments with this preparation (Munakata et al., 1988) demonstrated that increasing the tonicity of the solution perfusing the intraluminal surface of the trachea was a major stimulus for the release of EpDRF. 

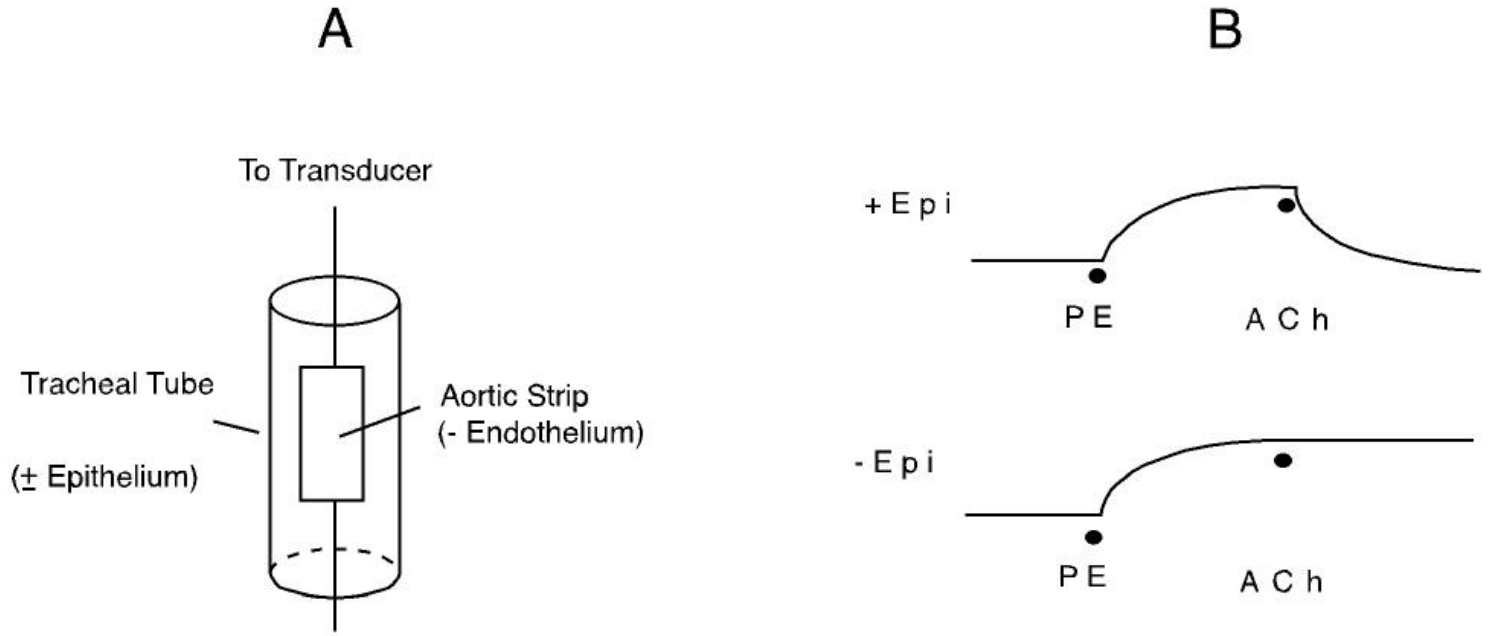

Figure 7. Coaxial bioassay preparation. (A) A diagram of the coaxial bioassay preparation whose use was first reported by Ilhan and Sahin in 1986. (B) A schematic illustration of the experimental results obtained from this preparation. The addition of $\mathrm{ACh}$ to an endotheliumdenuded, phenylephrine (PE)-contracted, rabbit aortic strip within an epithelium-intact (+Epi), guinea-pig trachea relaxed the aortic strip. When the same experiment was repeated using an epithelium-denuded (-Epi), guinea-pig trachea, the aortic strip failed to relax; thus, the ability of the epithelium to produce a transferrable EpDRF was concluded (illustration produced by and courtesy of Jeffrey S. Fedan, Ph.D.). 
When hypertonic solutions of $\mathrm{NaCl}, \mathrm{KCl}$, urea, or mannitol were added to the intraluminal surface of a carbachol-contracted trachea, the smooth muscle relaxed. When the experiment was repeated in epithelium-denuded trachea, smooth muscle relaxation was significantly attenuated, demonstrating that the relaxation response evoked by hypertonic solution was epitheliumdependent (Munakata et al., 1988). A separate study later demonstrated that increasing the tonicity of the extraluminal surface of the trachea also stimulated EpDRF-induced smooth muscle relaxation (Fedan et al., 1999). It has also been shown that stimulating the release of EpDRF with hypertonic solution decreased smooth muscle reactivity to methacholine and transmural nerve stimulation (Fedan et al., 1999). Although it has been shown that elevated extraluminal and intraluminal tonicity elicit epithelium-dependent smooth muscle relaxation via EpDRF, EpDRF's mechanism of action as well as its identity remains largely obscure.

It has been demonstrated clearly that EpDRF-induced smooth muscle relaxation, evoked by hypertonic solution, is unaffected by nitric oxide synthase inhibitors, indomethacin, methylene blue, propranolol, and tetrodotoxin (Munakata et al., 1988, 1990; Fedan et al., 1999). Interestingly, gossypol, a dual cyclooxygenase-lipoxygenase inhibitor and oxygen radical scavenger, and hemoglobin attenuate relaxation responses to elevated intraluminal tonicity (Teeter et al., 1988; Munakata et al., 1990; Fedan and Johnston, unpublished). Because hemoglobin, and not nitric oxide synthase inhibitors, attenuates EpDRF-induced smooth muscle relaxation, this suggests that carbon monoxide may play a role in the relaxation response to elevated tonicity since carbon monoxide is capable of binding to hemoglobin (West, 1995).

Previous work has suggested that the synthesis, release, and/or effects of EpDRF may be functionally linked to the electrical activity of epithelial cells (Raeburn and Fedan, 1989). Fedan et al. (1999) reported that amiloride-sensitive $\mathrm{Na}^{+}$channels and 4-4'-diisothiocyanatostilbene-2$2^{\prime}$ disulfonic acid (DIDS)-sensitive $\mathrm{Cl}^{\prime}$ channels seem to be involved in EpDRF-induced smooth muscle relaxation. Both amiloride and DIDS attenuate EpDRF-induced smooth muscle relaxation responses elicited by elevated intraluminal tonicity. Fedan et al. (1999) also reported that the $\mathrm{Na}^{+}-\mathrm{K}^{+}-2 \mathrm{Cl}^{!}$cotransporter and the $\mathrm{Na}^{+}-\mathrm{K}^{+}$-ATPase play virtually no role in EpDRF- 
induced smooth muscle relaxation. A separate study reported that epithelial $\mathrm{Ca}^{2+}$-activated $\mathrm{K}^{+}$ channels may be involved in the synthesis and/or release of EpDRF (Tamaoki et al., 1997). Dortch-Carnes et al. (1999) recently modified the isolated, perfused trachea apparatus to measure transepithelial potential difference simultaneously with smooth muscle contraction and relaxation. This modified preparation has provided more evidence that the synthesis, release, and/or effects of EpDRF are functionally linked to epithelial electrical activity. Dortch-Carnes et al. (1999) have reported that depolarization of the transepithelial potential difference precedes EpDRF-induced smooth muscle relaxation elicited by elevating intraluminal tonicity with either ionic or non-ionic osmolytes (Dortch-Carnes et al., 1999). A summary of EpDRF's actions are illustrated in Figure 8.

\section{Cell-Volume Regulation in Response to Hypertonic Challenge}

When exposed to anisotonic solutions, cells activate volume regulatory mechanisms to either maintain or return to their normal volume

(Parker, 1993; McManus et al., 1995; Haüssinger, 1996). When the tonicity of the mucosal and serosal surfaces of the airway epithelium are elevated, this is a major stimulus for the synthesis, release, and/or effects of EpDRF (Munakata et al., 1988; Fedan et al., 1999), it is therefore essential to understand the principles of cell-volume regulation as they pertain to hypertonic challenge. A cell loses water when exposed to a hypertonic environment. In order to regain its normal volume, the cell activates regulatory volume increase (RVI) mechanisms. Increases or decreases in cell volume can only be achieved by the gain or loss, respectively, of osmotically active solutes. Osmotically active solutes include inorganic ions such as $\mathrm{Na}^{+}, \mathrm{K}^{+}$, and $\mathrm{Cl}^{!}$and small, organic osmolytes. These small, organic osmolytes are divided into three major classes: (1) amino acids and their derivatives (i.e., alanine, proline, and taurine), (2) methylamines (i.e., betaine and glycerylphosphorylcholine), and (3) polyols (i.e., sorbitol and myo-inositol). These molecules are sometimes referred to as non-perturbing osmolytes since the cell can accumulate 
high concentrations of these substances without deleterious effects on cell structure and function (McManus et al., 1995; Lang et al., 1998). RVI mechanisms occur in two steps. First, within seconds after exposure to a hypertonic environment, a cell increases its net gain of $\mathrm{Na}^{+}$and $\mathrm{Cl}^{\text {! }}$ to maintain its volume through activation of the $\mathrm{Na}^{+}-\mathrm{K}^{+}-2 \mathrm{Cl}^{!}$cotransporter and the $\mathrm{Na}^{+}-\mathrm{H}^{+}$ exchanger, both of which are present on airway epithelial cells. Because the intracellular $\mathrm{pH}$ is altered by the activity of the $\mathrm{Na}^{+}-\mathrm{H}^{+}$exchanger, there is subsequent activation of the $\mathrm{Cl}^{!}-\mathrm{HCO}_{3}$ ! exchanger (Clarke and Boucher, 1993; McManus et al., 1995; Lang et al., 1998). 


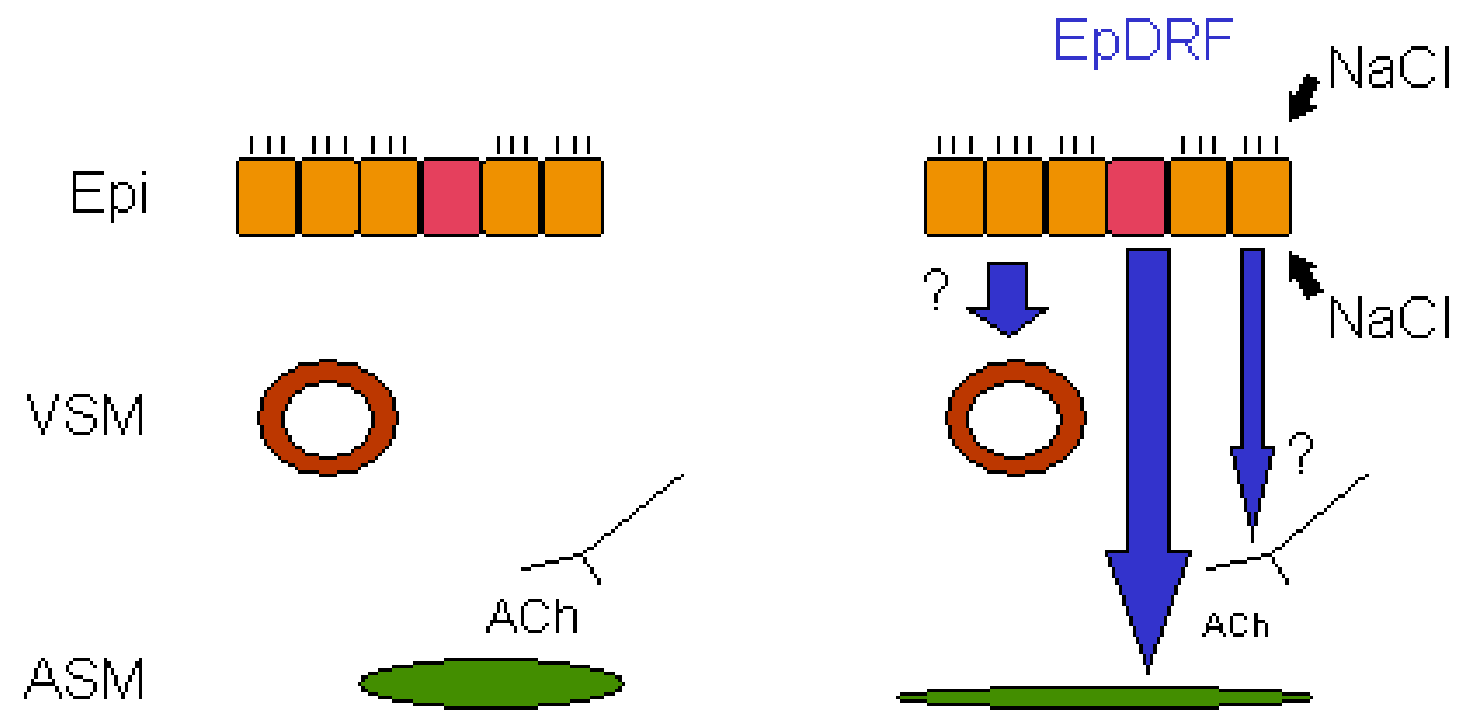

Figure 8. Actions of the airway epithelium-derived relaxing factor (EpDRF). EpDRF is known to be released when the tonicity of either the apical or basolateral surfaces of the airway epithelium are elevated, which in this illustration is denoted by $\mathrm{NaCl}$. Once released, EpDRF can relax the airway smooth muscle. It is still questionable whether or not EpDRF can relax vascular smooth muscle or if it is capable of attenuating the effects of ACh released from postganglionic parasympathetic nerve terminals. Epi, airway epithelium; VSM, vascular smooth muscle; ASM, airway smooth muscle; ACh, acetylcholine (illustration produced by and courtesy of Jeffrey S. Fedan, Ph.D.). 
The second step involves the accumulation of small organic osmolytes by activating the transcription of genes encoding osmolyte synthetic enzymes and transporters (Figure 9). These osmolytes are accumulated along with $\mathrm{Na}^{+}$by means of $\mathrm{Na}^{+}$-coupled osmolyte transporters, and this process can last for hours or days (McManus et al., 1995; Lang et al., 1998). In addition during RVI, cells breakdown cytoplasmic proteins while the anabolic pathways leading to the synthesis of organic osmolytes are increased (Haüssinger, 1996).

\section{Exercise-Induced Asthma}

In general, asthma can be defined as a chronic, inflammatory airway disease characterized by reversible airway obstruction. Asthma affects over $5 \%$ of the inhabitants of the world's industrialized countries (Shelhamer et al., 1995; Barnes et al., 1998). In the United States as many as twelve million persons may be afflicted with asthma (Shelhamer et al., 1995). The most common pathophysiological features of asthma include: (1) sloughing and desquamation of the respiratory epithelium, (2) thickening of the respiratory epithelial basement membrane, (3) goblet cell hyperplasia, (4) submucosal gland enlargement, (5) airway smooth muscle hypertrophy and hyperplasia, (6) dilation of the bronchial vasculature, (7) airway edema, (8) recruitment of inflammatory cells into the airways (Jeffrey, 1998), and (9) nonspecific airway hyperreactivity to nonsensitizing physical or chemical stimuli (Cockcroft and O'Byrne, 1993).

Approximately $70-80 \%$ of asthmatics experience exercise-induced asthma (EIA) which is a general term used to describe a transient increase in airway resistance following strenuous exercise. A decrease of $10 \%$ or more in the volume of air forcibly expired in one second $\left(\mathrm{FEV}_{1}\right)$ following exercise is used to diagnose EIA (Anderson, 1985; Anderson and Daviskas, 1999). 


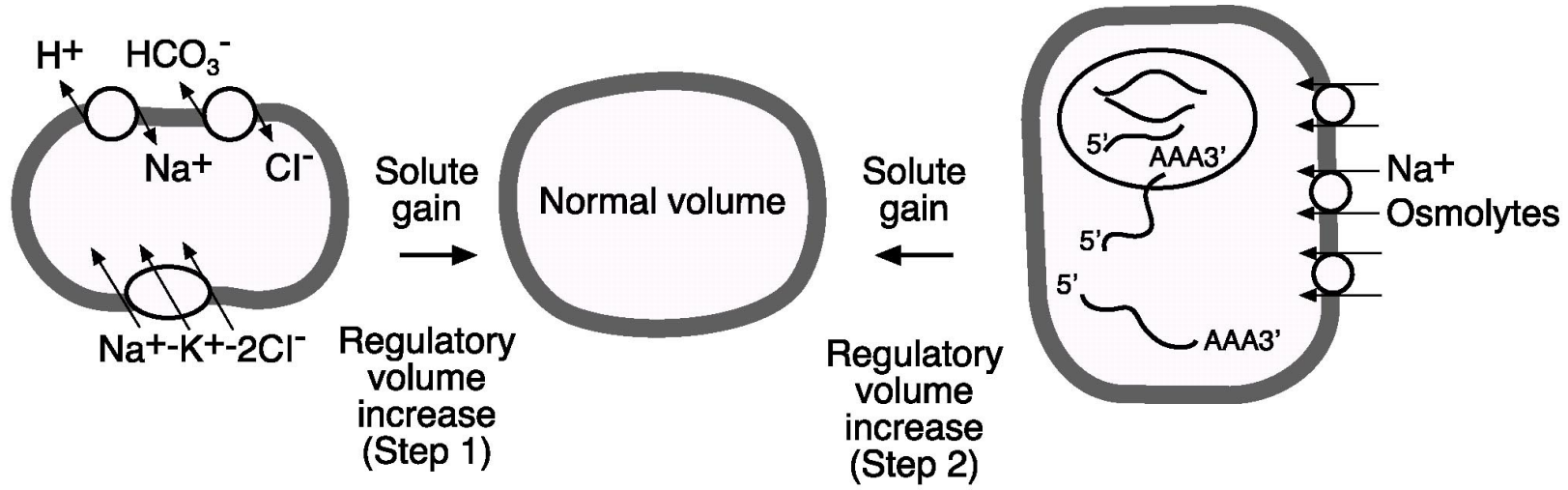

Figure 9. Mechanisms of regulatory volume increase (RVI). The RVI mechanisms occur in two steps. First, immediately after cells shrink due to exposure to a hypertonic environment, cells accumulate inorganic ions, namely $\mathrm{Na}^{+}$and $\mathrm{Cl}^{!}$through the activation of the $\mathrm{Na}^{+}-\mathrm{K}^{+}-2 \mathrm{Cl}$ ! cotransporter, the $\mathrm{Na}^{+}-\mathrm{H}^{+}$exchanger, and subsequently the $\mathrm{Cl}^{!}-\mathrm{HCO}_{3}$ ! exchanger. The second step occurs over many hours or days and involves the accumulation of organic osmolytes via the induction of genes encoding for osmolyte synthetic enzymes and ion-coupled transporters.

5'•AAA3' represents elevations in mRNA levels to convey an up-regulation of the transcription of genes encoding osmolyte synthetic enzymes and ion-coupled transporters. Figure adapted with permission from McManus ML, Churchwell KB and Strange K (1995) Regulation of cell volume in health and disease, $N$ Engl J Med 333:1260-1266. 
EIA is the most extreme five to ten minutes upon the cessation of exercise; however, a refractory period exists for up to three hours after the initial attack has ended (Anderson, 1985; McFadden, 1995). In contrast to asthmatics who may experience bronchoconstriction following exercise, normal individuals may actually experience bronchodilation during exercise (Kagawa and Kerr, 1970). Many hypotheses have tried to explain the phenomenon of EIA (Freed, 1995); however, two hypotheses in particular have gained the most attention: (1) the thermal hypothesis of EIA and (2) the osmotic theory of EIA. Most of the experimental evidence seems to support the osmotic theory of EIA (McFadden, 1995; Anderson and Daviskas, 1999) which is described in the following paragraphs.

Because of temperature and water vapor concentration gradients, heat and water are added to the inspired air as it passes over the respiratory mucosa of the conducting airways, a process referred to as conditioning. Under normal physiological conditions, most of the inspired air is conditioned by the time it reaches the oropharynx. During strenuous exercise, an individual's ventilation rate increases dramatically; therefore, the respiratory tract has to condition a greater volume of air over a much shorter period of time. As a consequence, more airways are actively recruited to condition the inspired air, and in the process, the respiratory mucosa loses more heat and water than normal to the inspired air. The ASL is the major source of water used to condition the inspired air during exercise. The water lost from the ASL during exercise is not readily replaced, and as a result, the osmolarity of the ASL increases (Anderson and Daviskas, 1999).

The osmotic theory of EIA suggests that the increased ASL osmolarity results in the release of bronchoactive mediators from the epithelium, bronchial mucosal mast cells, and sensory nerves which lead to EIA. Spasmogenic substances released from these cells include prostaglandin $\mathrm{D}_{2}$, leukotrienes, histamine, and substance $\mathrm{P}$, all of which can readily diffuse to the smooth muscle and initiate bronchoconstriction. Increasing the osmolarity of the ASL also leads to the release of bronchorelaxant substances from the airway epithelium, including NO, 
prostaglandin $\mathrm{E}_{2}$, and $\mathrm{EpDRF}$

(Munakata et al., 1988; Anderson and Daviskas, 1999; Fedan et al., 1999). The effects of airway drying on the airway epithelium and surrounding cells of the airway wall are illustrated in Figure 10.

The osmotic theory of EIA explains why some asthmatics could experience bronchoconstriction following exercise whereas normal individuals do not. In asthmatics, the airway epithelium is sloughed and desquamated (Laitinen et al., 1985), and as a consequence, more airways would have to be recruited to condition the inspired air. This could lead to an increase in the ASL osmolarity throughout the respiratory tract, resulting in the release of bronchospastic mediators from various sources to initiate EIA (Anderson and Daviskas, 1999). In normal individuals, the intact epithelium may produce enough bronchorelaxant factors $(\mathrm{NO}$, $\mathrm{PGE}_{2}$, and EpDRF) to offset the effects of these bronchospastic mediators; therefore EIA is prevented. 


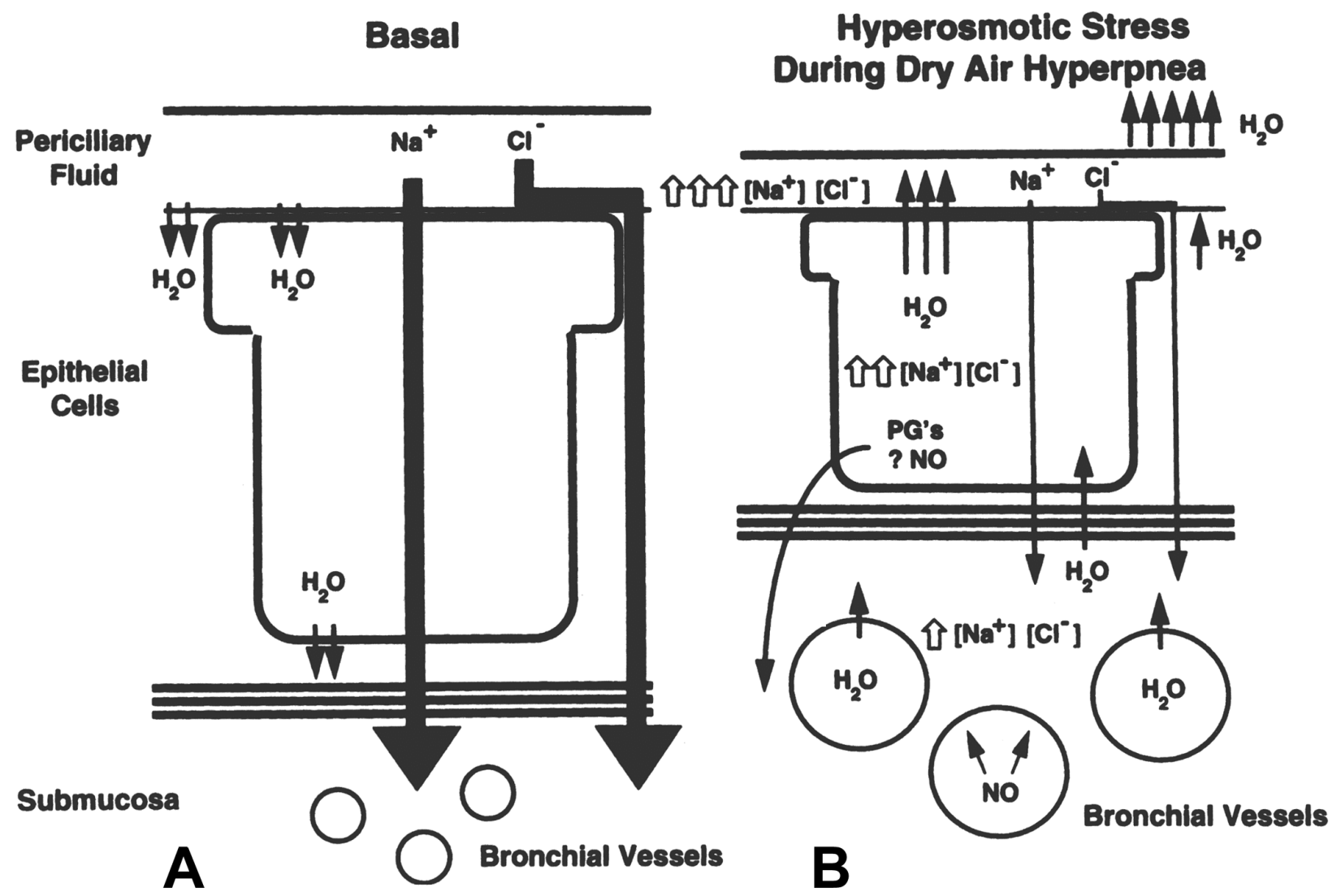

Figure 10. The effects of airway drying on the airway epithelium and airway wall. (A) The net direction of ion and water movement across the airway epithelium under normal, physiological conditions is indicated by the arrows. (B) Evaporative water loss (airway drying) as a result of hyperpnea reduces the depth of the periciliary fluid or ASL and increases its concentration of inorganic ions; thus, its osmolarity is elevated. This results in the release of bronchoactive mediators from the epithelium and the vascular endothelium of bronchial vessels in the airway wall. These mediators are capable of diffusing to the airway smooth muscle and modulating its tone. In addition, water movement from the submucosal tissues increases during this time in order to replenish the water lost from the periciliary fluid during airway drying. PGs, prostaglandins; NO, nitric oxide. Reprinted from Anderson SD and Daviskas E (1999) Airway drying and exercise-induced asthma, in Exercise-Induced Asthma (McFadden ER ed) pp 77-113 by courtesy of Marcel Dekker Inc. 


\section{Lipopolysaccharide, Sepsis, and Acute Respiratory Distress Syndrome}

Lipopolysaccharide (LPS) is an amphiphilic macromolecule which is the major component of the outer membrane of gram-negative bacteria (Figure 11A). LPS consists of two major structural components: a polysaccharide portion and a lipid A portion. The lipid A component is covalently bound on one side to the outer membrane of the bacterium whereas the polysaccharide portion is bound to the other end of the lipid A component and extends into the extracellular space. As Figure 11B illustrates, the polysaccharide portion can be further subdivided into a core region and an $\mathrm{O}$-specific chain. The lipid A portion is highly conserved among gram-negative bacteria and is the toxic component of the LPS macromolecule. As gramnegative bacteria die or begin to replicate, LPS is released and capable of initiating its pathological effects (Lüderitz et al., 1982; Rietschel et al., 1994, 1996; Raetz, 1996).

LPS, in itself, is not toxic, but it is the immunologic and inflammatory events that it initiates which are detrimental. Various serum proteins are capable of modulating the effects of LPS, but the most important is the lipopolysaccharide-binding protein (LBP). By binding to the LBP, the LPS macromolecule is delivered to a membrane-bound receptor, CD14, found on macrophages and monocytes, and once bound, LPS-induced signal transduction can commence (Rietschel et al., 1996). LPS stimulates a wide variety of cellular processes, some of which are summarized in Figure 12. An overwhelming gram-negative bacterial infection can result in sepsis and/or acute respiratory distress syndrome (ARDS). 

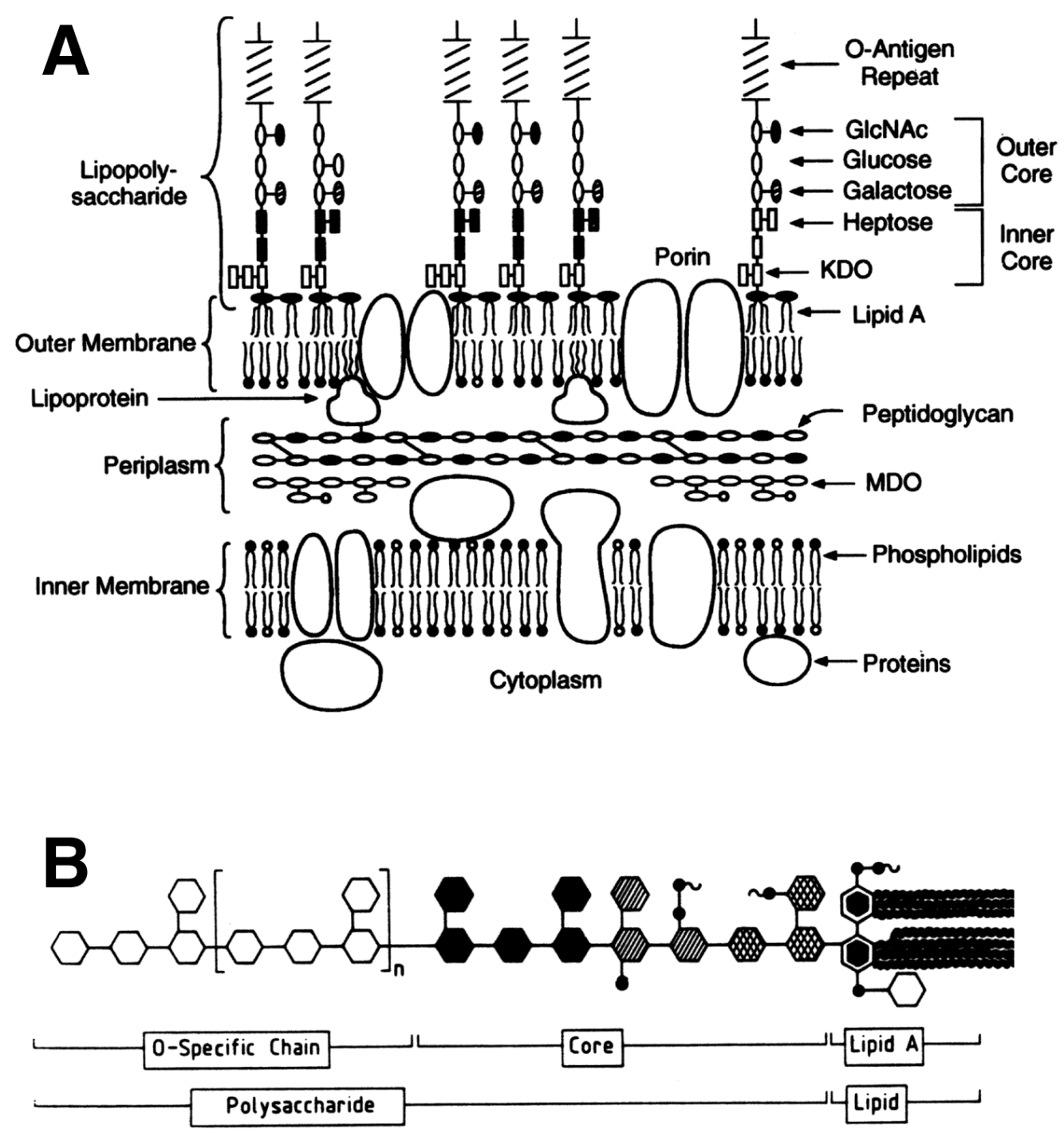

$\square$ :Monosaccharide, $\bullet$ :Phosphate, 〜 :Ethanolamine 〜 Long Chain(Hydroxy) Fatty Acid

Figure 11. Localization of lipopolysaccharide (LPS) and its chemical components. (A) LPS is the major component of the outer membrane of gram-negative bacteria. GluNAc, N-acetyl-Dglucosamine; KDO, 3-deoxy-D-manno-octulosonic acid; MDO, membrane-derived oligosaccharides. Reprinted with permission from Raetz CRH (1996) Bacterial lipopolysaccharides: A remarkable family of bioactive macroamphiphiles, in Escherichia coli and Salmonella: Cellular and molecular biology (Neidhardt FC ed) pp 1035-1063, ASM Press, Washington, D.C. (B) The LPS macromolecule is divided into two major regions: the lipid A portion and the polysaccharide portion. The polysaccharide region can be further subdivided. Reprinted with permission from, Academic Press Ltd, London UK. 


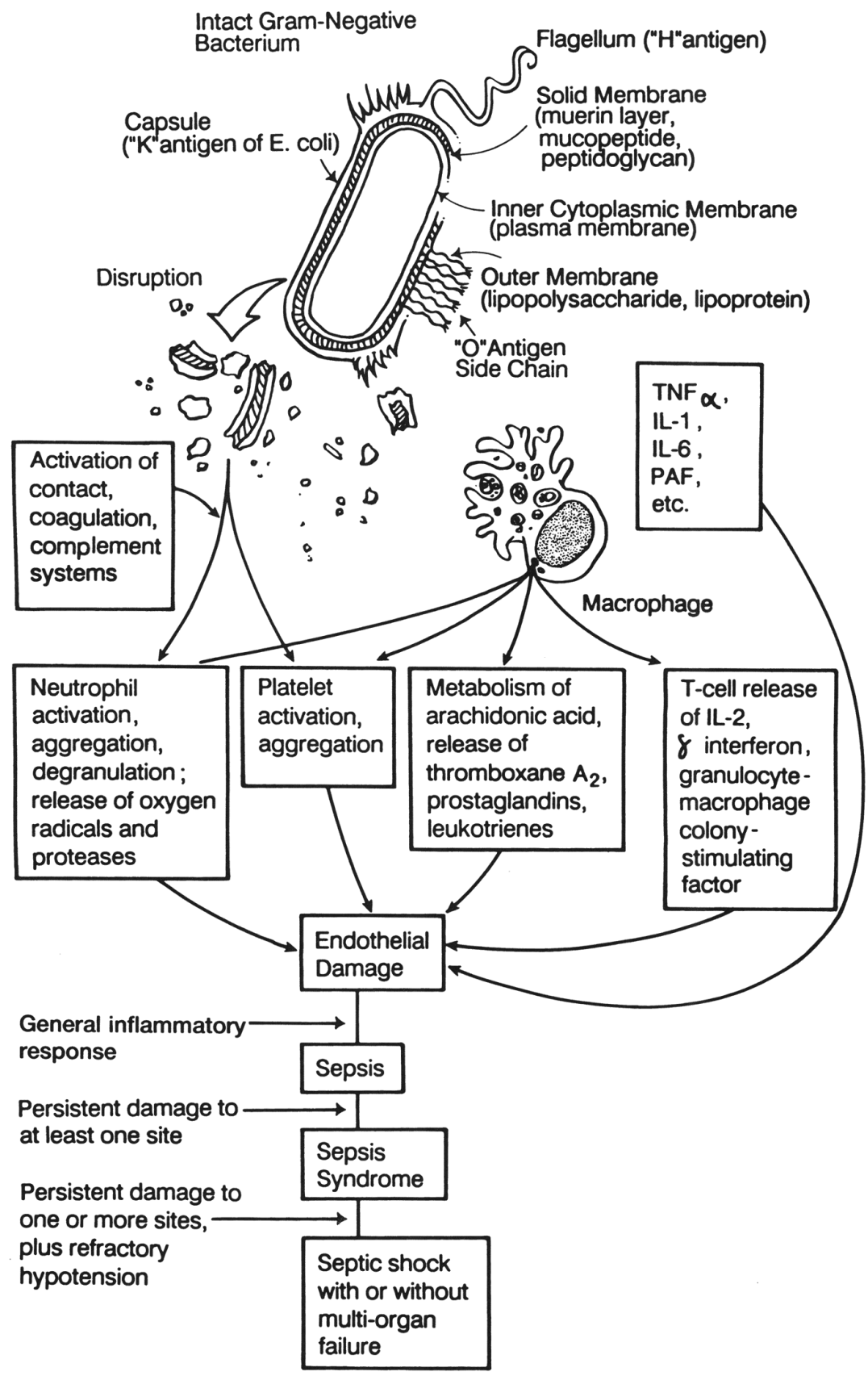

Figure 12. Toxic effects of lipopolysaccharide (LPS). As gram-negative bacteria begin to replicate or die, LPS is released and capable of exerting its pathological effects. If a gramnegative bacterial infection is severe enough, it can result in sepsis. TNF", tumor necrosis factor "; IL-1, interleukin-1; IL-6, interleukin-6; PAF, platelet-activating factor; IL-2, interleukin-2. Reprinted with permission from Bone RC (1991) The pathogenesis of sepsis, Ann Int Med 115(6):457-469. 
As defined by the American College of Chest Physicians/Society of Critical Care Medicine, sepsis is the systemic response to infection (Bone, 1996), and as many as 500,000 new cases are reported annually in the United States (Sessler et al., 1996). Sepsis is a serious medical condition with a 40\% mortality rate (Marsh and Wewers, 1996). A few of the clinical manifestations of sepsis include fever, hypothermia, tachycardia, tachypnea, oliguria, acidosis, and hypotension (Rietschel et al., 1996). Fifty percent of those individuals diagnosed with sepsis develop ARDS within the first twenty-four hours after the onset of sepsis. Acute lung injury is recognized clinically as ARDS (Sessler et al., 1996). The clinical manifestations of ARDS include arterial hypoxemia, dyspnea, decreased lung compliance, pulmonary edema, and atelectasis (McFeely and Hudson, 1994; Sessler et al., 1996).

This dissertation describes an investigation into two separate, but related questions pertaining to the role and mechanism(s) of action of EpDRF. In the first, the effects of epithelial ion transport inhibitors on the epithelial bioelectric response to elevated mucosal tonicity were examined in order to elucidate which ion channels and/or transporters are involved in the epithelial depolarization which precedes EpDRF-induced smooth muscle relaxation. In the second study, the effects of LPS-treatment on EpDRF-induced smooth muscle relaxation and related bioelectric events were examined, since LPS has been implicated in a number of pulmonary diseases. 


\section{The Relationship Between Epithelial Bioelectric Responses and Epithelium-Derived Relaxing Factor- \\ Induced Airway Smooth Muscle Relaxation in Response to Hypertonic Solution}




\section{Objectives}

When cells are exposed to a hypertonic environment, they activate RVI mechanisms which alter ion transport through perturbations in ion channel and/or transporter activity (McManus et al., 1995; Lang et al., 1998). In addition, when the extraluminal (serosal) and intraluminal (mucosal) surfaces of guinea-pig tracheal epithelium are challenged with hypertonicity, EpDRF is released (Munakata et al., 1988; Fedan et al., 1999), an event which seems to be functionally linked to epithelial ion transport (Dortch-Carnes et al., 1999; Fedan et al., 1999). With a consideration of these statements, the purpose of this study was to characterize further the epithelial bioelectric events associated with the synthesis, release, and/or effects of EpDRF in guinea-pig isolated trachea.

The hypothesis underlying this study was that hypertonicity-induced alterations in epithelial ion transport are functionally linked to the synthesis, release, and/or effects of EpDRF. The hypothesis was tested by measuring transepithelial short-circuit current $\left(I_{\mathrm{sc}}\right)$ responses to pharmacological agents added to either surface of guinea-pig isolated trachea mounted in Ussing chambers. Dortch-Carnes et al. (1999) have shown that EpDRF-induced smooth muscle relaxation is preceded by a depolarization of the transepithelial potential difference while Fedan et al. (1999) have shown that EpDRF-induced smooth muscle relaxation is significantly attenuated by amiloride ( $\mathrm{Na}^{+}$channel blocker) and DIDS ( $\mathrm{Cl}^{!}$channel blocker). The experiments in this study will try to determine which epithelial ion channels and/or transporters are involved in the transepithelial depolarization preceding EpDRF-induced airway smooth muscle relaxation. This will be accomplished by examining the effect of various ion channel and transporter inhibitors on $I_{\mathrm{sc}}$ responses to hypertonic modified Krebs Henseleit (MKH) solution. In this study, the MKH solution was made hypertonic by adding either $120 \mathrm{mOsm} \mathrm{NaCl}$ or Dmannitol to the MKH solution. 


\section{Materials and Methods}

\section{Animals}

Specific, pathogen-free, male Dunkin-Hartley guinea pigs (Harlan Sprague Dawley, Inc.; Indianapolis, IN; 370-714 g) were used during the course of this study. The guinea pigs were housed in separate, actively ventilated, micro-isolator cages (Lab Products, Inc.; Seaford, DE) within a single room. The room within which these animals were housed were part of an 8800 $\mathrm{ft}^{2}$ modified barrier, animal care facility. Within this facility, the animals were acclimated to their new environment prior to experimentation, given food and water ad libitum, and breathed fresh air. All of the animals in the animal care facility, as well as the facility itself, were under the supervision of a full-time veterinarian.

Throughout this study, the guinea pigs were sacrificed according to a standard protocol. The animals were first anesthetized intraperitoneally (i.p.) with sodium pentobarbital (Veterinary Laboratories, Inc.; Lenexa, KS; $65 \mathrm{mg} / \mathrm{kg}$ ), and once it was determined that the animals were unresponsive to any stimuli, they were exsanguinated by severing the abdominal aorta and cutting the heart.

All of the experimental protocols in this study were approved by the Institutional Animal Care and Use Committee (IACUC) while the facilities where the experimental protocols were conducted were fully accredited by the Association for the Assessment and Accreditation of Laboratory Animal Care (AAALAC) International. These facilities have been accredited by AAALAC International since February 18, 1987.

\section{Ussing System}

A classical Ussing system [World Precision Instruments (WPI); Sarasota, FL] was utilized to directly measure $I_{\mathrm{sc}}$ in freshly isolated, guinea-pig tracheal segments. $I_{\mathrm{sc}}$ can be defined as the current required to offset the spontaneous transepithelial potential difference 
generated by the tissue. Under $I_{\mathrm{sc}}$ conditions there is no net ion transport; therefore, $I_{\mathrm{sc}}$ is a measurement of total active ion transport (Soybel, 1994).

The Ussing system consisted of two separate, water-jacketed, glass circulation reservoirs whose temperature was maintained at $37^{\circ} \mathrm{C}$. The reservoirs were filled with recirculating, $\mathrm{MKH}$ solution, a physiological salt solution, which was maintained at $37^{\circ} \mathrm{C}$ and continuously gassed with $95 \% \mathrm{O}_{2}-5 \% \mathrm{CO}_{2}$. The reservoirs were connected to opposing hemi-chambers, between which a guinea-pig tracheal segment was mounted. Because of the manner in which the tissue was mounted, pharmacological agents could be added selectively to either the mucosal or serosal surfaces of the trachea.

In order to prepare a guinea-pig tracheal segment for mounting, the animal was sacrificed as previously described. The trachea was exposed in vivo, the entire trachea excised, placed in MKH solution gassed with $95 \% \mathrm{O}_{2}-5 \% \mathrm{CO}_{2}$, and cleaned of extraneous tissue. Once cleaned, the trachea was slit along its longitudinal axis by cutting through the airway smooth muscle. The trachea was then opened as a flat sheet and cut horizontally so that the proximal end of the trachea was mounted in the Ussing chamber. After mounting, only a $0.125 \mathrm{~cm}^{2}$ surface area of the trachea was exposed to the gassed, MKH solution from the glass circulation reservoirs.

The fluid which bathed the mucosal and serosal surfaces of the trachea was in contact with silver/silver chloride electrode cartridges (WPI; Sarasota, FL) which were filled with $0.9 \%$ sodium chloride irrigation, USP (saline; Baxter Healthcare Corporation; Deerfield, IL) in 4 \% melted bacteriological agar. A two-channel DVC-1000 voltage/current clamp (WPI; Sarasota, FL) was connected to the Ussing chamber via four silver/silver chloride electrodes. Two electrodes, placed distal to the tissue, were connected to the current-passing circuit while two electrodes, placed proximal to the tissue, monitored transepithelial potential difference. $I_{\mathrm{sc}}$ was measured continuously, and the data recorded on a conventional strip chart recorder (Gould Instrument Systems; Valley View, $\mathrm{OH})$. In addition, the $I_{\mathrm{sc}}$ was normalized to surface area. To accomplish this, the $I_{\mathrm{sc}}$ was multiplied by the inverse of the surface area of the trachea exposed to 
the fluid bathing the mucosal and serosal surfaces. In this instance, the surface area was calculated from the formula describing the surface area of a circle:

$$
\text { surface area of a circle }=\mathrm{Br}^{2}
$$

where $\mathrm{r}$ is the radius $(0.2 \mathrm{~cm})$. The following sample calculation demonstrates how the $I_{\mathrm{sc}}$ was eventually expressed in $\mu \mathrm{A} / \mathrm{cm}^{2}$ :

$$
I_{\mathrm{sc}} \times\left(1 / \mathrm{Br}^{2}\right)
$$

then, by substitution of arbitrary numerical values

$$
5.0 \mu \mathrm{A} \times 1 /\left[\mathrm{B}(0.2 \mathrm{~cm})^{2}\right]=39.8 \mu \mathrm{A} / \mathrm{cm}^{2}
$$

The tissue was equilibrated for $3 \mathrm{hr}$ prior to any experimental manipulations, with the MKH solution bathing the mucosal and serosal surfaces of the trachea being changed every 15 min.

\section{Administration of Agents to the Ussing System}

The Ussing system was used in this study to determine the effect of amiloride $\left(\mathrm{Na}^{+}\right.$

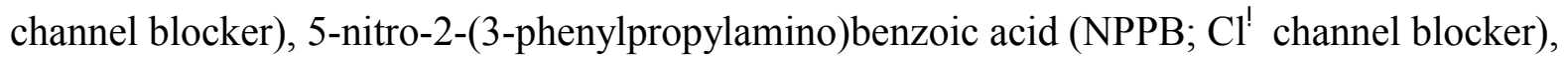
iberiotoxin $\left(\mathrm{Ca}^{2+}\right.$-activated $\mathrm{K}^{+}$channel blocker $)$, bumetanide $\left(\mathrm{Na}^{+}-\mathrm{K}^{+}-2 \mathrm{Cl}^{\text {! }}\right.$ cotransporter inhibitor), and ouabain $\left(\mathrm{Na}^{+}-\mathrm{K}^{+}\right.$-ATPase inhibitor) on tracheal $I_{\mathrm{sc}}$ responses to hypertonic solution. During this portion of the study, the effect of a single concentration of hypertonic solution on $I_{\mathrm{sc}}$ was examined, and in this instance, the MKH solution was made hypertonic by adding either120 mOsm of $\mathrm{NaCl}$ or D-mannitol to the MKH solution.

Following the $3 \mathrm{hr}$ equilibration period, hypertonic $\mathrm{NaCl}$ or D-mannitol solution was added to the mucosal surface of the trachea in the presence of serosally-added methacholine chloride $\left(\mathrm{MCh} ; 3 \times 10^{! 7} \mathrm{M}\right)$ in order to mimic conditions in previous EpDRF studies (Dortch-Carnes et al., 1999; Fedan et al., 1999). Once the electrophysiological response to hypertonic solution had equilibrated, the preparation was then washed at 15 min intervals for 90 min with fresh MKH solution. 
After the completion of the $90 \mathrm{~min}$ wash period, a respective ion channel and/or transporter inhibitor was added to either the mucosal or serosal surface of the trachea, depending upon the epithelial surface where its molecular target is believed to exist (Figure 6). Once the electrophysiological response of the inhibitor, if any, had equilibrated, hypertonic $\mathrm{NaCl}$ or $\mathrm{D}$ mannitol solution was added again to the mucosal surface of the trachea in the presence of serosally-added MCh $\left(3 \times 10^{! 7} \mathrm{M}\right)$. There were a few exceptions to this standard protocol. Iberiotoxin was added to both the mucosal and serosal surfaces of the trachea for 30 min while ouabain was added prior to the addition of MCh to mimic conditions in a previous EpDRF study, which was done to prevent $\mathrm{Na}^{+}$loading of the tracheal smooth muscle (Fedan et al., 1999).

\section{Solutions and Reagents}

The MKH solution contained (mM): $\mathrm{NaCl}$ (113.0), $\mathrm{KCl}(4.8), \mathrm{CaCl}_{2}(2.5), \mathrm{KH}_{2} \mathrm{PO}_{4}$ (1.2), $\mathrm{MgSO}_{4}$ (1.2), $\mathrm{NaHCO}_{3}$ (25.0), and glucose (5.7). The MKH solution was continuously gassed with $95 \% \mathrm{O}_{2}-5 \% \mathrm{CO}_{2}$ and maintained at $37^{\circ} \mathrm{C}$ and a $\mathrm{pH}$ of 7.4 . In addition, the osmolarity of the MKH solution was 281.2 $\pm 0.6 \mathrm{mOsm}(n=5$; Osmette A Automatic Osmometer, Precision Systems, Inc.; Sudbury, MA).

All chemical reagents used during the course of this study were purchased from Sigma Chemical Co. (St. Louis, MO) and dissolved in distilled water unless otherwise noted. Amiloride and ouabain were dissolved in warm, distilled water while bumetanide and NPPB were dissolved in dimethyl sulfoxide (DMSO).

\section{Analysis of Results}

When statistically comparing the $\triangle I_{\mathrm{sc}}$ in response to hypertonic MKH solution in the presence and absence of ion channel or transporter inhibitors, the paired Student's $t$ test or MannWhitney rank sum test was used, as appropriate. 
All of the results were expressed as the mean \pm S.E.M. (standard error of the mean) where $n$ is the number of separate experiments. In every experiment, $p<0.05$ was considered significant.

\section{Results}

\section{Effect of ion transport inhibitors on the basal $I_{\text {sc }}$ of isolated trachea}

The basal $I_{\mathrm{sc}}$ of guinea-pig isolated, trachea mounted in Ussing chambers was $47.8 \pm 1.6$ $\mu \mathrm{A} / \mathrm{cm}^{2}(n=64)$. Treatment of the tissue with mucosally-applied amiloride $\left(3 \times 10^{! 5} \mathrm{M}\right)$, mucosally-applied NPPB $\left(10^{! 4} \mathrm{M}\right)$, or serosally-applied bumetanide $\left(10^{! 5} \mathrm{M}\right)$ decreased the basal $I_{\mathrm{sc}}$ by $35.6 \pm 1.4 \%(n=12), 83.3 \pm 4.9 \%(n=8)$, and $8.9 \pm 1.6 \%(n=12)$, respectively. Mucosally- and serosally-applied iberiotoxin $\left(10^{! 7} \mathrm{M}\right)$ had no apparent effect upon the basal $I_{\text {sc }}$. In order to mimic previous studies of EpDRF in the isolated, perfused trachea apparatus (Fedan et al., 1999), the effect of serosally-applied ouabain $\left(10^{! 5} \mathrm{M}\right)$ on $I_{\mathrm{sc}}$ was measured in the presence of serosally-applied MCh $\left(3 \times 10^{! 7} \mathrm{M}\right)$. In this instance, ouabain decreased the $I_{\mathrm{sc}}$ by $94.3 \pm 3.3 \%$ $(n=8)$

\section{Effect of ion transport inhibitors on the bioelectric responses of isolated trachea to hypertonic $\mathrm{NaCl}$ and D-mannitol solution}

In order to characterize further the ion channels and/or transporters involved in EpDRFinduced smooth muscle relaxation (Dortch-Carnes et al., 1999; Fedan et al., 1999), this study examined the effect of ion channel and transporter inhibitors on the $I_{\mathrm{sc}}$ responses of isolated trachea to $\mathrm{MKH}$ solution made hypertonic with either added $\mathrm{NaCl}$ (120 mOsm) or D-mannitol (120 mOsm), both of which have been shown to elicit EpDRF-induced smooth muscle relaxation (Munakata et al., 1988; Fedan et al., 1999). Again, in order to mimic previous studies of EpDRF (Dortch-Carnes et al., 
1999; Fedan et al., 1999), MCh $\left(3 \times 10^{! 7} \mathrm{M}\right)$ was added serosally prior to the mucosal application of hypertonic $\mathrm{NaCl}$ or D-mannitol solution. $\mathrm{MCh}$, itself, increased the $I_{\mathrm{sc}}$ which was significantly attenuated by bumetanide and NPPB (data not shown).

In the presence of serosally-added MCh $\left(3 \times 10^{! 7} \mathrm{M}\right)$, the bioelectric responses to hypertonic $\mathrm{NaCl}$ and D-mannitol solution were different (Figure 13). Mucosally-applied hypertonic $\mathrm{NaCl}$ solution produced a triphasic response which consisted of a rapid decrease, an increase, and finally, a very slow, sustained decrease in $I_{\mathrm{sc}}$. The triphasic $I_{\mathrm{sc}}$ responses were difficult to quantify since the first two phases, the rapid decrease and then increase, were variable from tissue to tissue; however, the very slow, sustained decrease in $I_{\mathrm{sc}}$ was characteristic of all tissue responses to hypertonic $\mathrm{NaCl}$ solution. As a consequence, the very slow, sustained decrease in $I_{\mathrm{sc}}$ was the only phase which was quantified. The response to hypertonic D-mannitol solution was much less complex, consisting of only a very slow, sustained decrease in $I_{\mathrm{sc}}$.

Pretreatment with either serosally-applied amiloride $\left(3 \times 10^{! 5} \mathrm{M}\right)$ or bumetanide $\left(10^{! 5} \mathrm{M}\right)$ significantly inhibited the $\triangle I_{\mathrm{sc}}$ induced by $\mathrm{NaCl}$ and D-mannitol (Figures 14 and 15) while mucosally- and serosally-applied iberiotoxin $\left(10^{! 7} \mathrm{M}\right)$ had no apparent effect on the $I_{\mathrm{sc}}$ responses to either $\mathrm{NaCl}$ or D-mannitol (Figure 16$)$. In the presence of mucosally-applied NPPB $\left(10^{! 4} \mathrm{M}\right)$, the $I_{\mathrm{sc}}$ increased rather than decreased following the addition of $\mathrm{NaCl}$ while the $\triangle I_{\mathrm{sc}}$ induced by D-mannitol was almost completely abolished (Figure 17). Serosally-added ouabain $\left(10^{!} \mathrm{M}\right)$ caused a progressive reduction in $I_{\mathrm{sc}}$ toward zero. This prevented assessment of the effect of ouabain on responses to $\mathrm{NaCl}$ and D-mannitol; however, in the presence of ouabain, the $I_{\mathrm{sc}}$ increased rather than decreased following the application of $\mathrm{NaCl}$ and $\mathrm{D}$-mannitol (Figure 18). 

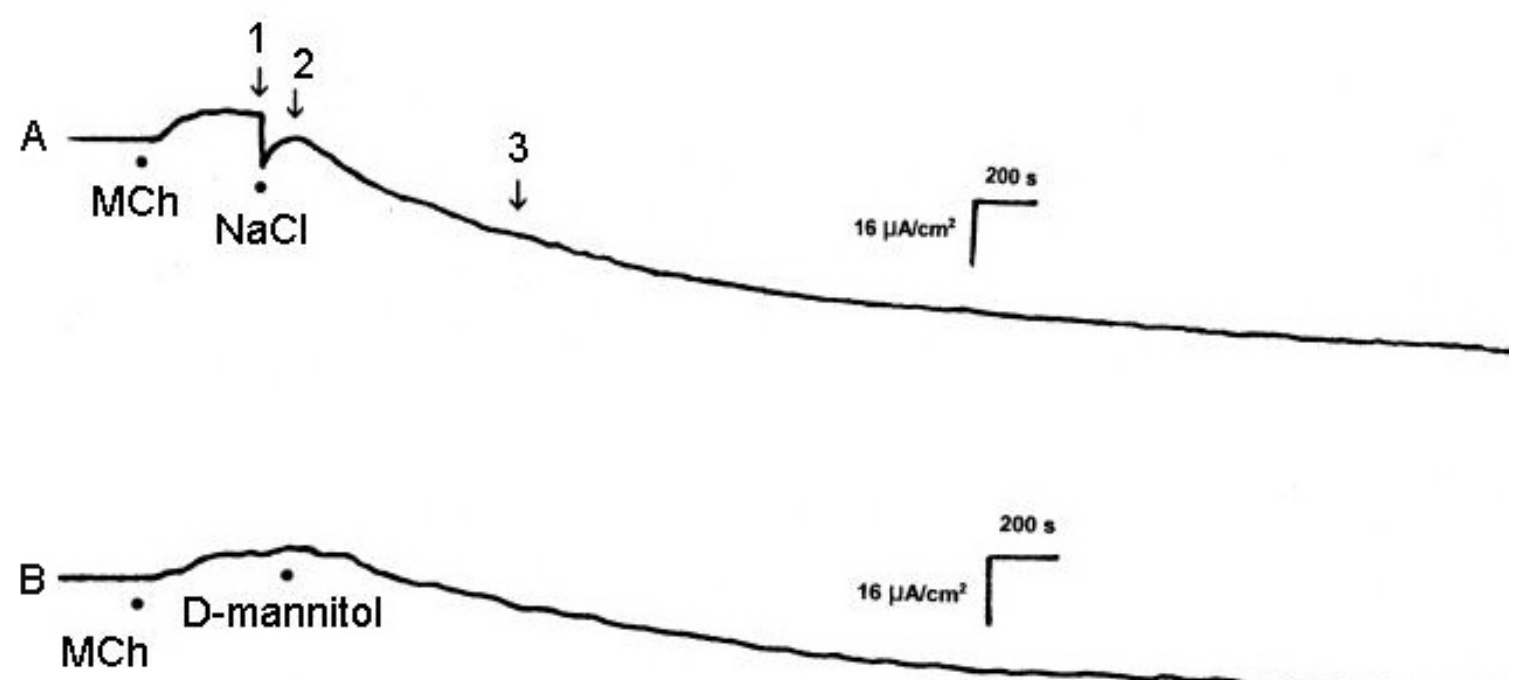

Figure 13. Representative tracing showing the effect of mucosally-applied MKH solution made hypertonic with added (A) $\mathrm{NaCl}(120 \mathrm{mOsm})$ or (B) D-mannitol $(120 \mathrm{mOsm})$ on $I_{\mathrm{sc}}$ in the presence of serosally-applied $\mathrm{MCh}\left(3 \times 10^{! 7} \mathrm{M}\right)$ in isolated trachea. MCh increased the $I_{\mathrm{sc}} . \mathrm{NaCl}$ elicited a triphasic response which consisted of (1) a rapid decrease, (2) an increase, and finally, (3) a very slow, sustained decrease in $I_{\text {sc. }}$. D-mannitol elicited a very slow, sustained decrease in $I_{\mathrm{sc}}$. As explained in the text, it was the very, slow sustained decrease in $I_{\mathrm{sc}}$ which was quantified. 


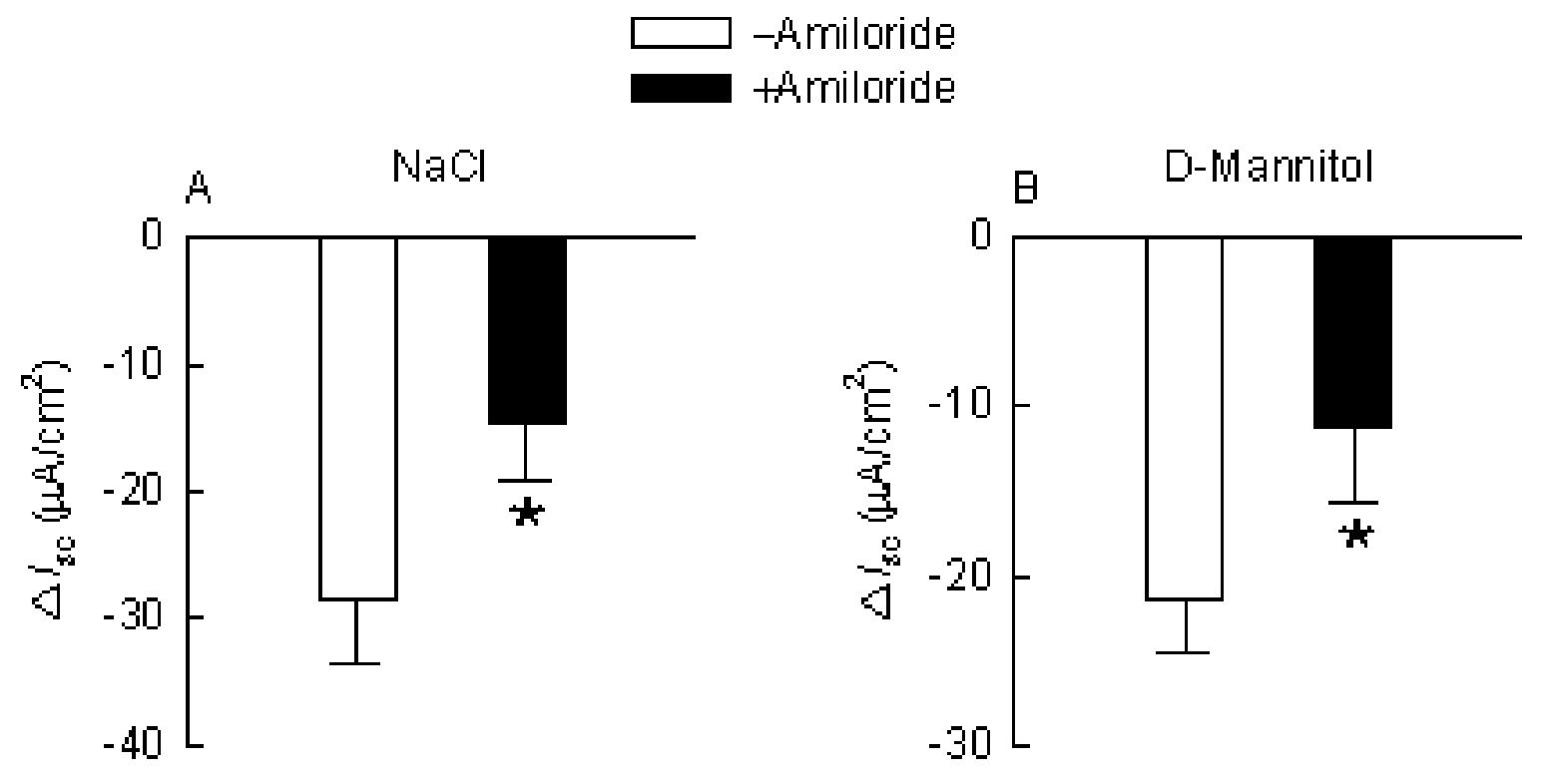

Figure 14. Effect of mucosally-applied amiloride $\left(3 \times 10^{! 5} \mathrm{M}\right)$ on the $I_{\mathrm{sc}}$ responses of mucosallyapplied MKH solution made hypertonic with added (A) $\mathrm{NaCl}$ (120 mOsm) or (B) D-mannitol $(120 \mathrm{mOsm})$ in the presence of serosally-applied $\mathrm{MCh}\left(3 \times 10^{! 7} \mathrm{M}\right)$ in isolated trachea.

Amiloride significantly inhibited the $\triangle I_{\mathrm{sc}}$ induced by both $\mathrm{NaCl}$ and D-mannitol. $n=6$ for $\mathrm{NaCl}$ and D-mannitol. * $p<0.05$ compared to ! Amiloride as determined by a paired Student's $t$ test. 


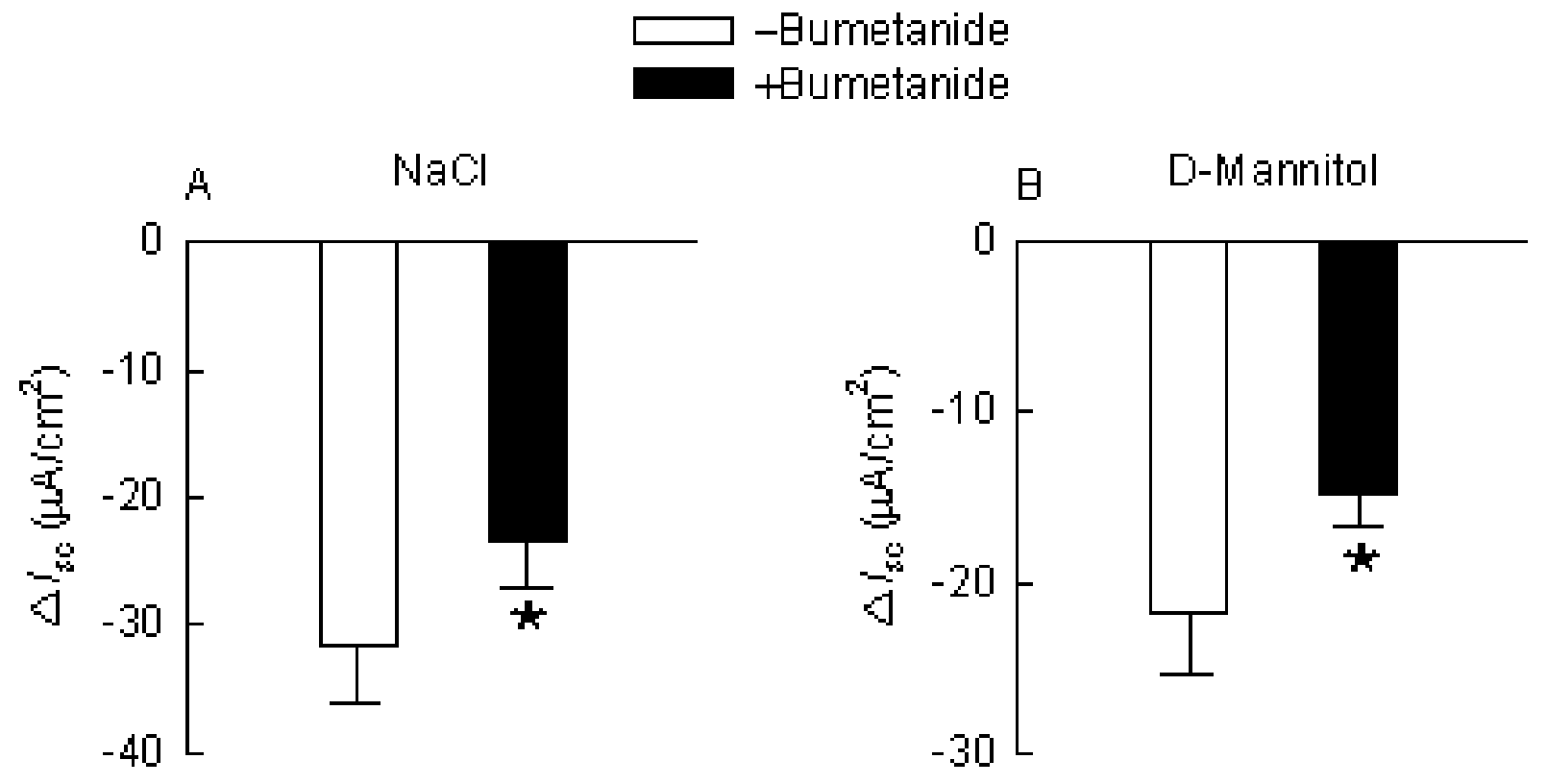

Figure 15. Effect of serosally-applied bumetanide $\left(10^{! 5} \mathrm{M}\right)$ on the $I_{\mathrm{sc}}$ responses of mucosallyapplied MKH solution made hypertonic with added (A) $\mathrm{NaCl}$ (120 mOsm) or (B) D-mannitol $(120 \mathrm{mOsm})$ in the presence of serosally-applied $\mathrm{MCh}\left(3 \times 10^{! 7} \mathrm{M}\right)$ in isolated trachea.

Bumetanide significantly inhibited the $\triangle I_{\mathrm{sc}}$ induced by both $\mathrm{NaCl}$ and $\mathrm{D}$-mannitol. $n=6$ for $\mathrm{NaCl}$ and D-mannitol. ${ }^{*} p<0.05$ compared to ! Bumetanide as determined by a paired Student's $t$ test. 


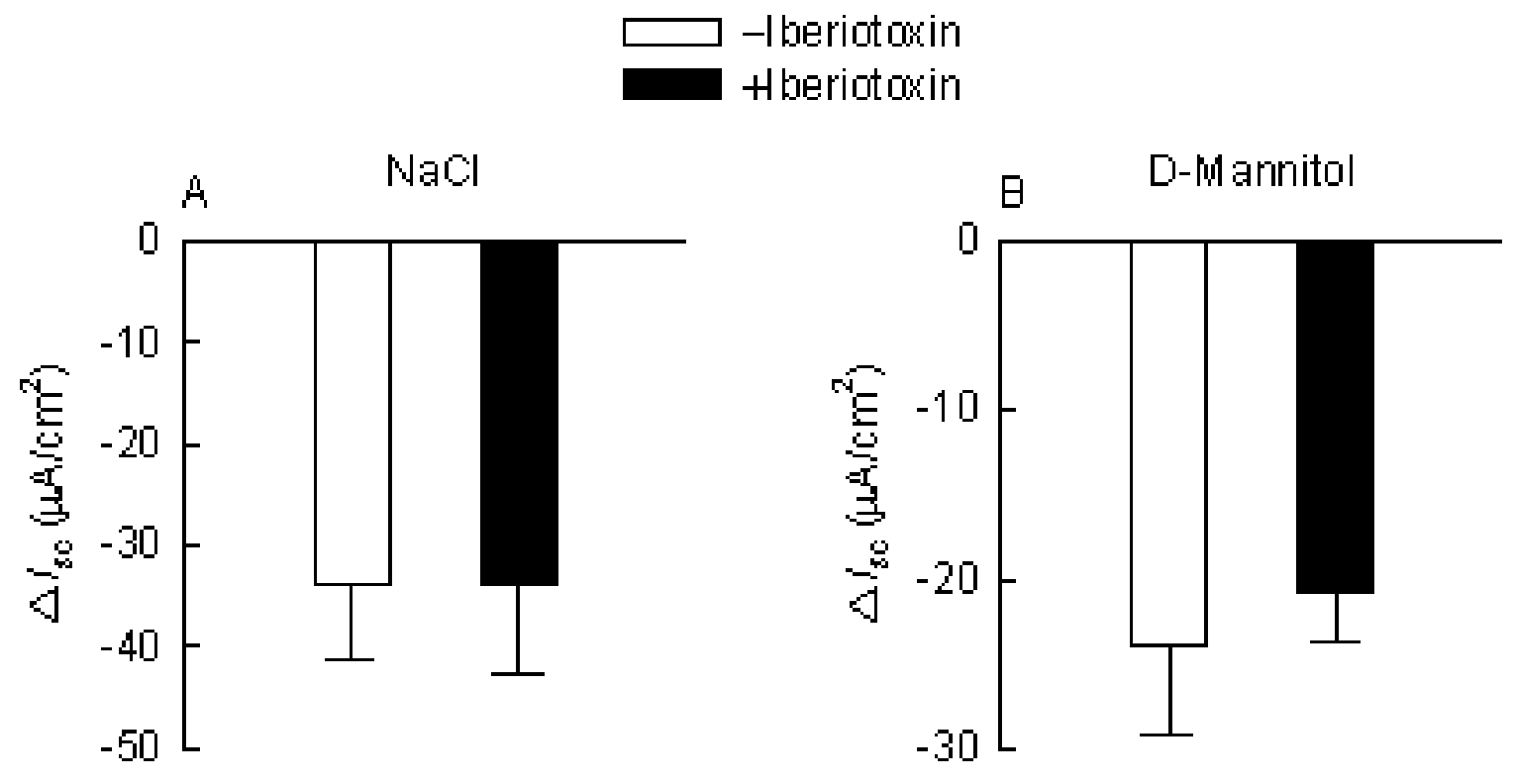

Figure 16. Lack of effect of mucosally- and serosally-applied iberiotoxin $\left(10^{!} \mathrm{M}\right)$ on the $I_{\mathrm{sc}}$ responses of mucosally-applied $\mathrm{MKH}$ solution made hypertonic with added (A) $\mathrm{NaCl}(120$ mOsm) or (B) D-mannitol $(120 \mathrm{mOsm})$ in the presence of serosally-applied MCh $\left(3 \times 10^{! 7} \mathrm{M}\right)$ in isolated trachea. $n=4$ for $\mathrm{NaCl}$ and D-mannitol. 


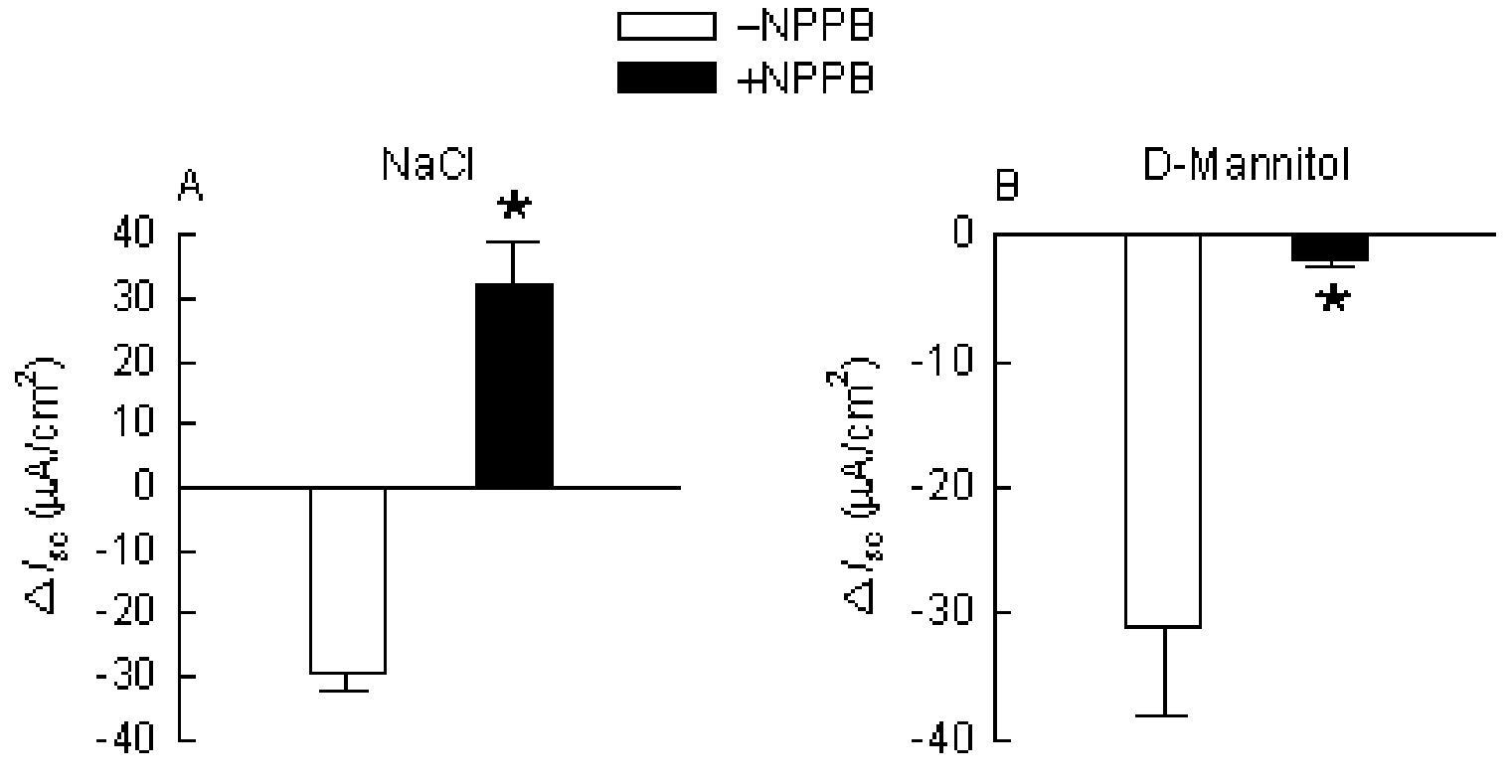

Figure 17. Effect of mucosally-applied NPPB $\left(10^{! 4} \mathrm{M}\right)$ on the $I_{\mathrm{sc}}$ responses of mucosallyapplied MKH solution made hypertonic with added (A) NaCl (120 mOsm) or (B) D-mannitol $(120 \mathrm{mOsm})$ in the presence of serosally-applied $\mathrm{MCh}\left(3 \times 10^{! 7} \mathrm{M}\right)$ in isolated trachea. In the presence of NPPB, the $I_{\mathrm{sc}}$ increased rather than decreased following the addition of $\mathrm{NaCl}$ while the $\triangle I_{\text {sc }}$ induced by D-mannitol was almost completely abolished. $n=4$ for $\mathrm{NaCl}$ and Dmannitol. ${ }^{*} p<0.05$ compared to ! NPPB as determined by a paired Student's $t$ test. 


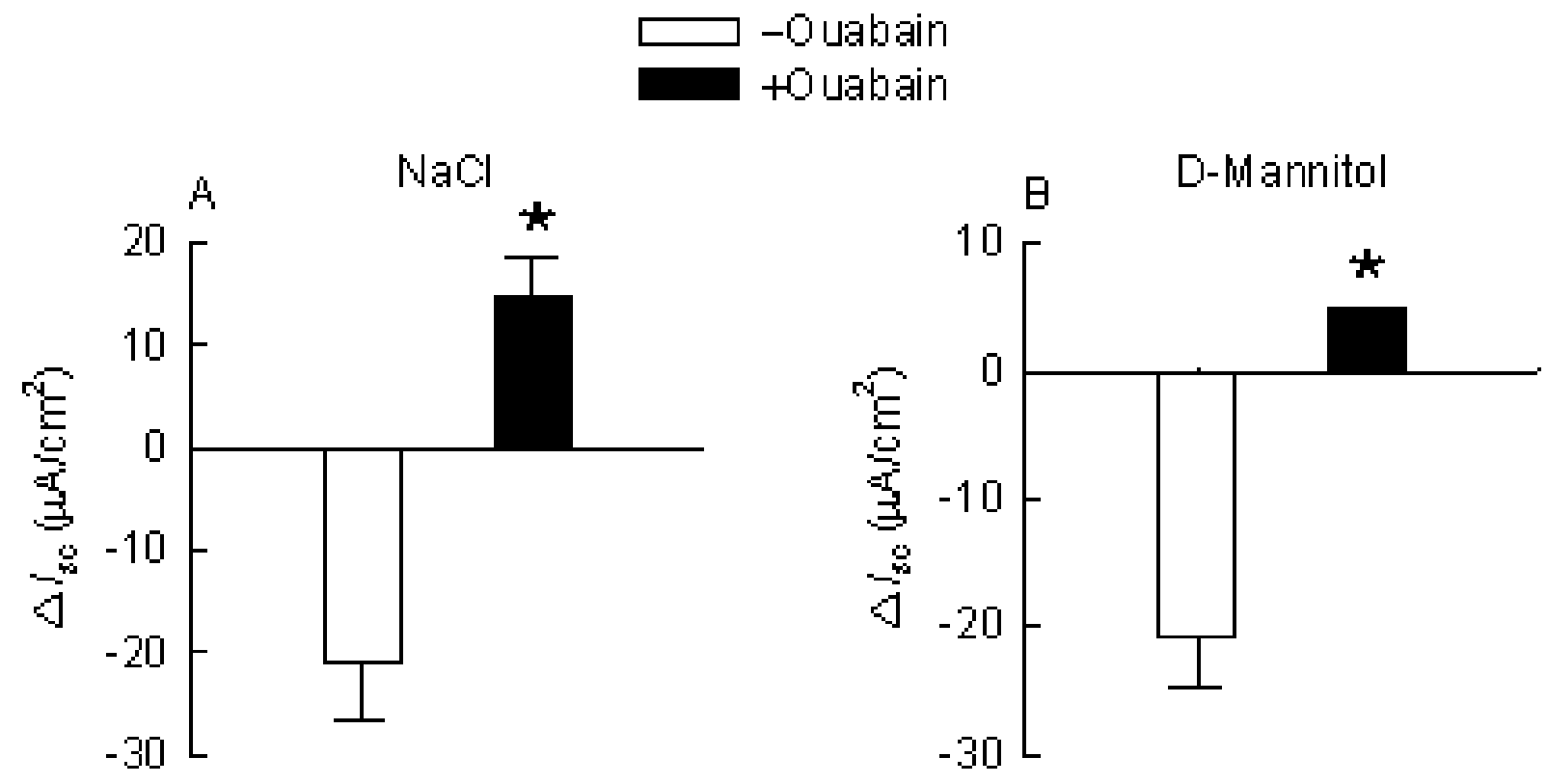

Figure 18. Effect of serosally-applied ouabain $\left(10^{! 5} \mathrm{M}\right)$ on the $I_{\mathrm{sc}}$ responses of mucosallyapplied MKH solution made hypertonic with added (A) $\mathrm{NaCl}$ (120 mOsm) or (B) D-mannitol $(120 \mathrm{mOsm})$ in the presence of serosally-applied MCh $\left(3 \times 10^{! 7} \mathrm{M}\right)$ in isolated trachea. Ouabain $\left(10^{! 5} \mathrm{M}\right)$ caused a progressive reduction in $I_{\mathrm{sc}}$ toward zero. This prevented assessment of the effect of ouabain on responses to $\mathrm{NaCl}$ and $\mathrm{D}$-mannitol; however, in the presence of ouabain, the $I_{\mathrm{sc}}$ increased rather than decreased following the application of $\mathrm{NaCl}$ and D-mannitol. $n=4$ for $\mathrm{NaCl}$ and D-mannitol. ${ }^{*} p<0.05$ compared to ! Ouabain as determined by a paired Student's $t$ test. 
In order to make certain that the responses seen in the presence of the inhibitors were not due to the effects of the vehicle, DMSO, the effect of DMSO on the responses was tested. DMSO had no effect upon the responses to hypertonic $\mathrm{NaCl}$ or D-mannitol solution (Figure 19). Finally, to ensure that tissue desensitization did not contribute to the apparent effect of the inhibitors, the effect of hypertonic $\mathrm{NaCl}$ and $\mathrm{D}$-mannitol solution on $I_{\mathrm{sc}}$ responses was measured twice in the same preparation (Figure 20). The second response was measured 90 min after the first, with washes every $15 \mathrm{~min}$. There was no difference between the first and second response to $\mathrm{D}$-mannitol, but there was a significant decrease in the second $\mathrm{NaCl}$ response when compared to the first. 


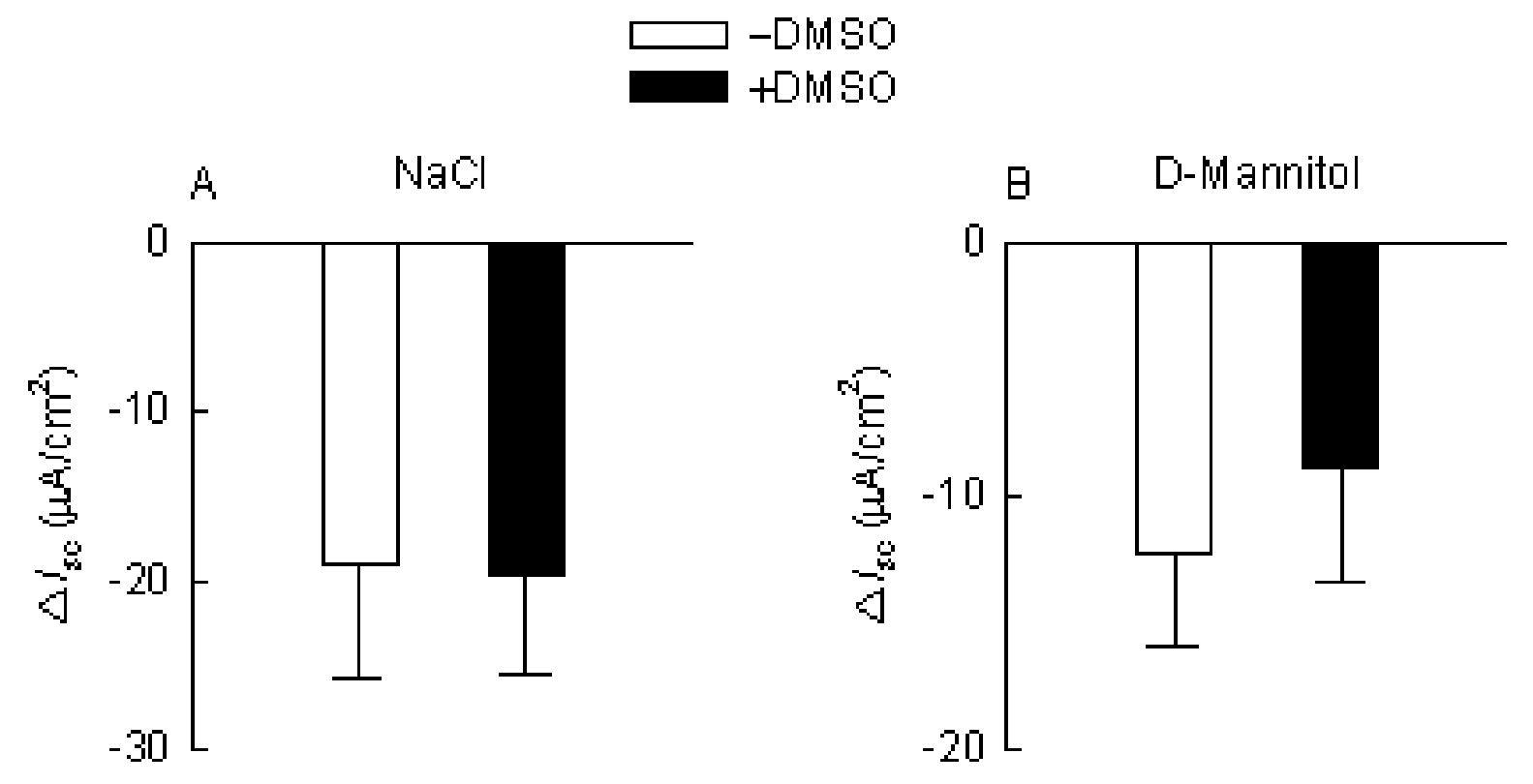

Figure 19. Lack of effect of mucosally- and serosally-applied DMSO on the $I_{\mathrm{sc}}$ responses of mucosally-applied MKH solution made hypertonic with added (A) $\mathrm{NaCl}$ (120 mOsm) or (B) Dmannitol $(120 \mathrm{mOsm})$ in the presence of serosally-applied $\mathrm{MCh}\left(3 \times 10^{! 7} \mathrm{M}\right)$ in isolated trachea. The final concentration of DMSO bathing the mucosal surface of the trachea was $0.08 \%$ while the final concentration bathing the serosal surface of the trachea was $0.01 \% . n=4$ for $\mathrm{NaCl}$ and D-mannitol. 


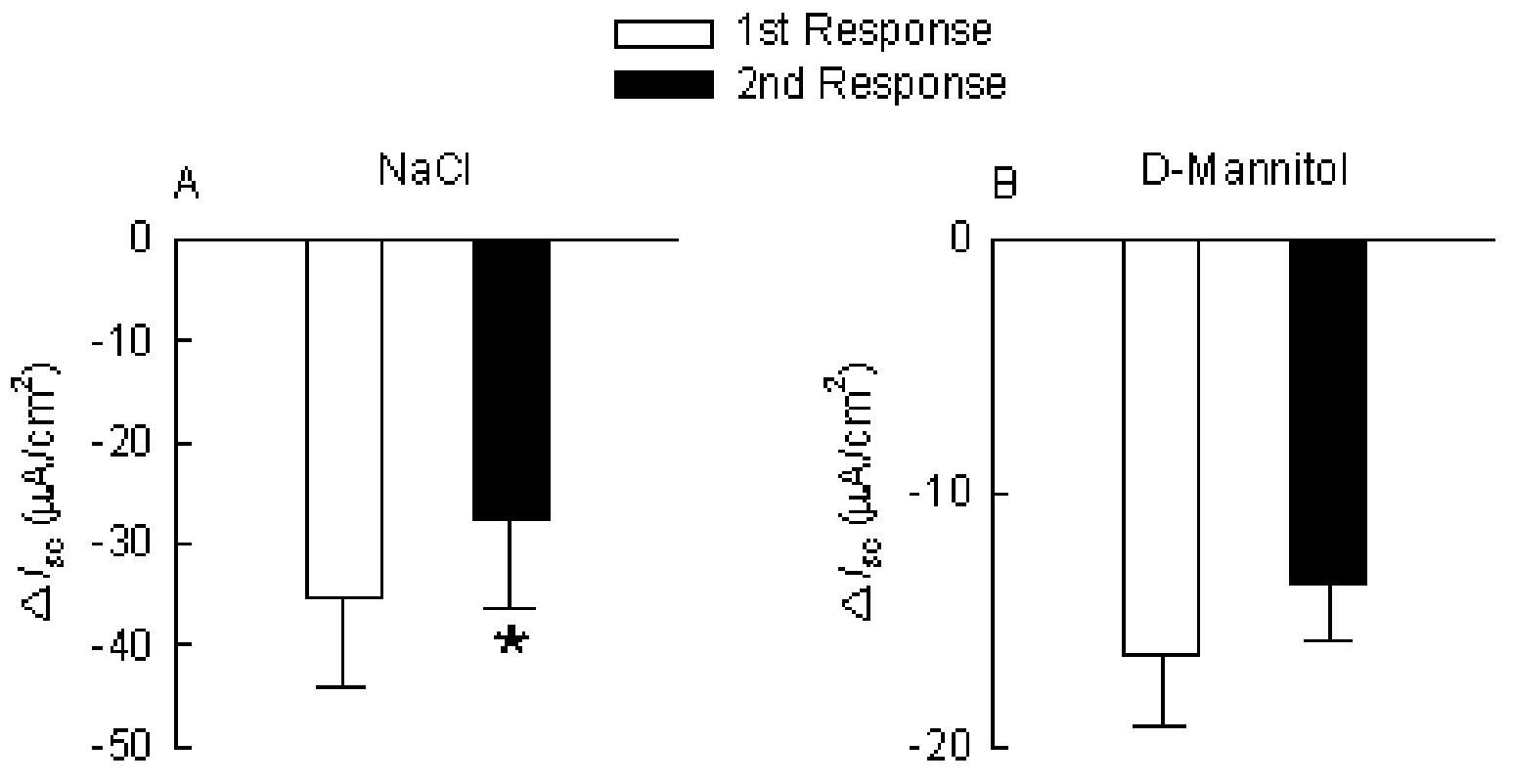

Figure 20. Comparison of the first and second $I_{\mathrm{sc}}$ responses to mucosally-applied MKH solution made hypertonic with added (A) $\mathrm{NaCl}(120 \mathrm{mOsm})$ or (B) D-mannitol (120 mOsm) in the presence of serosally-applied MCh $\left(3 \times 10^{! 7} \mathrm{M}\right)$ in isolated trachea. The second $\triangle I_{\mathrm{sc}}$ induced by $\mathrm{NaCl}$ was slightly, but significantly less than the first while there was no difference between the first and second $\triangle I_{\mathrm{sc}}$ induced by D-mannitol. $n=4$ for $\mathrm{NaCl}$ and D-mannitol. ${ }^{*} p<0.05$ compared to the first response as determined by a paired Student's $t$ test. 


\section{Discussion}

The results of this study suggest that apical $\mathrm{Na}^{+}$channels and basolateral $\mathrm{Na}^{+}-\mathrm{K}^{+}-2 \mathrm{Cl}^{\text {! }}$ cotransporters are intimately involved in the airway epithelial bioelectric response to elevated mucosal tonicity while the role of apical $\mathrm{Cl}^{!}$channels and basolateral $\mathrm{Na}^{+}-\mathrm{K}^{+}$-ATPases is unclear. Since apical $\mathrm{Na}^{+}$channels are involved in both the epithelial bioelectric response to elevated mucosal tonicity and hypertonicity-induced smooth muscle relaxation via EpDRF, this further strengthens the hypothesis that the synthesis, release, and/or effects of EpDRF are functionally linked to the electrical activity of the epithelium (Raeburn and Fedan, 1989; DortchCarnes et al., 1999; Fedan et al., 1999).

Although the major objective of this study was to elucidate which epithelial ion channels and/or transporters were involved in the depolarization of the transepithelial potential difference $\left(\mathrm{V}_{\mathrm{ms}}\right)$ preceding EpDRF-induced smooth muscle relaxation, this study has made other observations regarding the basic electrophysiological properties of guinea-pig tracheal epithelium. The basal $I_{\mathrm{sc}}$ value obtained in this study was not consistent with two previous studies measuring $I_{\mathrm{sc}}$ in guinea-pig tracheal epithelium (Croxton, 1993; Robison and Kim, 1994). The reasons for these discrepancies are not clear; however, both of these studies used animals in a lower weight range which may be important if epithelial ion transport is size- and/or agedependent. More importantly, the difference may lie in the arrangement of the tissue. Croxton (1993) measured $I_{\mathrm{sc}}$ in intact trachea while Robison and Kim (1994) measured $I_{\mathrm{sc}}$ in cultured, tracheobronchial epithelial cells. This is most significant when comparing our results with those from cultured cells since submucosal tissues have been shown to modulate the bioelectric properties of the airway epithelium (Freed and Croxton, 1993). The lack of an airway wall and its modulatory effects in cultured cells may explain these differences. Our basal $I_{\mathrm{sc}}$ value $\left(47.8 \pm 1.6 \mu \mathrm{A} / \mathrm{cm}^{2}\right)$, however, was consistent with a separate study $\left(37.3 \pm 2.9 \mu \mathrm{A} / \mathrm{cm}^{2}\right)$ which also measured $I_{\mathrm{sc}}$ in guinea-pig tracheal segments mounted in Ussing chambers (Stutts and Bromberg, 1987). 
The effects of several ion transport inhibitors on the basal $I_{\text {sc }}$ produced results which contradicted the current, presumed model of ion transport in guinea-pig tracheal epithelium. The current model proposes that under resting $I_{\mathrm{sc}}$ conditions $\mathrm{Na}^{+}$absorption is the major ion transport process which accounts for the majority of the $I_{\text {sc }}$ (Boucher et al., 1982). In this study, amiloride decreased the basal $I_{\mathrm{sc}}$ by $35.6 \pm 1.4 \%$ which is consistent with a $\mathrm{Na}^{+}$absorptive epithelium; however, other studies have shown the effect of amiloride on baseline $I_{\mathrm{sc}}$ to be much greater. Croxton (1993) reported that amiloride $\left(3 \times 10^{! 5} \mathrm{M}\right)$ decreased the baseline $I_{\mathrm{sc}}$ by approximately $50 \%$ while Robison and Kim (1994) reported that amiloride $\left(10^{! 5} \mathrm{M}\right)$ decreased the baseline $I_{\mathrm{sc}}$ by approximately $75 \%$. Again, these discrepancies may be explained by the same reasons given for the differences seen in the basal $I_{\mathrm{sc}}$ among the different preparations.

Our results became more contradictory when the effect of NPPB, a $\mathrm{Cl}^{!}$channel blocker, on baseline $I_{\mathrm{sc}}$ was examined. NPPB abolished $83.3 \pm 4.9 \%$ of the basal $I_{\mathrm{sc}}$. Despite the fact that the current model of ion transport in guinea-pig tracheal epithelium proposes that there is no net $\mathrm{Cl}^{!}$secretion (Boucher et al., 1982), our results with NPPB suggest that $\mathrm{Cl}^{!}$secretion may be the predominant ion transport process of this epithelium. Robison and Kim (1994) used another $\mathrm{Cl}^{\text {! }}$ channel blocker, N-phenylanthranilic acid, which decreased the basal $I_{\mathrm{sc}}$ of cultured, tracheobronchial epithelial cells by approximately $20 \%$. On the surface, the effect of NPPB suggests that the majority of the $I_{\mathrm{sc}}$ is due to $\mathrm{Cl}^{!}$secretion; however, NPPB is a nonselective $\mathrm{Cl}^{\text {! }}$ channel blocker. In fact, NPPB has been shown to inhibit the $\mathrm{Ca}^{2+}$ release-activated $\mathrm{Ca}^{2+}$ channel as well as the activity of the $\mathrm{Na}^{+}-\mathrm{K}^{+}$-ATPase which may account for its large inhibition of baseline $I_{\text {sc }}$ (Ito et al., 2000; Li et al., 2000).

With respect to the effect of hypertonic solution on $I_{\mathrm{sc}}$ responses in the presence of serosally-added MCh $\left(3 \times 10^{! 7} \mathrm{M}\right)$, the results of this study demonstrate that these responses are dependent upon the osmolyte used to elevate mucosal tonicity. The triphasic change in $I_{\mathrm{sc}}$ following the addition of hypertonic $\mathrm{NaCl}$ solution is very intriguing; however, the electrophysiological basis of the first two phases, the rapid decrease followed by an increase, could not be elucidated because these particular responses were inconsistent between and within 
animals. In response to $\mathrm{MKH}$ solution made hypertonic with either $\mathrm{NaCl}$ or $\mathrm{D}$-mannitol, there was always a slow, sustained decrease in $I_{\mathrm{sc}}$, indicating that this electrophysiological response is the characteristic bioelectric response of the airway epithelium to elevated mucosal tonicity.

Since MKH solution made hypertonic with either added $\mathrm{NaCl}$ or D-mannitol elicited a slow, sustained decrease in $I_{\mathrm{sc}}$, the effect of ion transport inhibitors on this particular response was evaluated. This decrease in $I_{\mathrm{sc}}$ following an elevation in mucosal tonicity may be explained by the same mechanisms believed to account for hyperosmotic stress of the airways during dry air hyperpnea (Figure 10; Anderson and Daviskas, 1999). Because elevated mucosal tonicity would decrease the osmotic pressure of the ASL, water would exit the cell; thus, intracellular $\mathrm{Na}^{+}$and $\mathrm{Cl}^{!}$concentrations would increase. Consequently, this would decrease $\mathrm{Na}^{+}$absorption and increase $\mathrm{Cl}^{\text {! }}$ secretion. A decrease in $\mathrm{Na}^{+}$absorption and an increase in $\mathrm{Cl}^{\text {! }}$ secretion would decrease and increase the $I_{\mathrm{sc}}$ respectively, and the net effect on $I_{\mathrm{sc}}$ would be dependent upon which ion transport process dominates. In addition, the effect on $I_{\mathrm{sc}}$ elicited by elevated mucosal tonicity is also dependent upon the paracellular pathway conductance. A previous study reported that the decrease in $I_{\mathrm{sc}}$ following elevated mucosal tonicity was due to a decrease in $\mathrm{Na}^{+}$ absorption. In fact, this same study also reported that $\mathrm{Cl}^{!}$secretion was not induced by elevated mucosal tonicity (Willumsen et al., 1994). This explanation describing the decrease in $I_{\mathrm{sc}}$ fits with the effects of D-mannitol since it is membrane-impermeable; however, the effects of $\mathrm{NaCl}$ are more complicated because it is membrane-semipermeable. In order to fully elucidate the particular ion fluxes which are altered following exposure of the tracheal epithelium to elevated mucosal tonicity, isotope flux studies are necessary.

The significant attenuation of the hypertonicity-induced $\triangle I_{\mathrm{sc}}$ by amiloride, regardless of the osmolyte used to elevated tonicity, suggests that apical $\mathrm{Na}^{+}$channels are involved in the epithelial bioelectric response to elevated mucosal tonicity. In the presence of amiloride, the $I_{\mathrm{sc}}$ response to elevated mucosal tonicity is most likely reduced for two reasons. First, $\mathrm{Na}^{+}$is unable to leave the cell across the apical membrane in the presence of amiloride. Secondly, amiloride 
stimulates $\mathrm{Cl}^{\text {! }}$ secretion (Boucher et al., 1982), which would offset any decrease in $I_{\mathrm{sc}}$ by hypertonic solution.

The significant, inhibitory effect of bumetanide on hypertonicity-induced $\triangle I_{\mathrm{sc}}$, regardless of the osmolyte used to elevate tonicity, suggests that basolateral $\mathrm{Na}^{+}-\mathrm{K}^{+}-2 \mathrm{Cl}^{!}$cotransporters along with apical $\mathrm{Na}^{+}$channels are involved in the epithelial bioelectric response to elevated mucosal tonicity. The reason bumetanide attenuates the $\triangle I_{\mathrm{sc}}$ elicited by elevated mucosal tonicity is dependent upon the existence and activation of another basolateral $\mathrm{Cl}^{\text {! }}$-coupled transporter when the $\mathrm{Na}^{+}-\mathrm{K}^{+}-2 \mathrm{Cl}^{!}$cotransporter is inhibited. Two candidates include the $\mathrm{Cl}^{!}$$\mathrm{HCO}_{3}{ }^{!}$exchanger and the $\mathrm{Na}^{+}$-coupled $\mathrm{Cl}^{!}$transporter. In fact, the $\mathrm{Na}^{+}$-coupled $\mathrm{Cl}^{!}$transporter is postulated to exist in the basolateral membranes of $\mathrm{Cl}^{!}$secretory epithelia (Frizzell et al., 1979). How would the action of the $\mathrm{Na}^{+}$-coupled $\mathrm{Cl}^{!}$transporter, if it exists in guinea-pig tracheal epithelium, offset the decrease in $I_{\mathrm{sc}}$ elicited by elevated mucosal tonicity? According to the model of hyperosmotic stress of the airways during dry air hyperpnea, not only do the intracellular concentrations of $\mathrm{Na}^{+}$and $\mathrm{Cl}^{!}$increase, but also the extracellular concentrations of these ions in the submucosa (Figure 10) (Anderson and Daviskas, 1999) which would tend to drive these ions into the overlying epithelial cell by means of the $\mathrm{Na}^{+}$-coupled $\mathrm{Cl}^{\text {! }}$ transporter if the $\mathrm{Na}^{+}-\mathrm{K}^{+}-2 \mathrm{Cl}^{!}$cotransporter is inhibited by bumetanide. $\mathrm{Na}^{+}$and $\mathrm{Cl}^{!}$would begin to accumulate in the cell, and in order to obtain electrochemical equilibrium, they must be extruded across either the apical or basolateral membranes. According to the model proposed by Frizzell et al. (1979), $\mathrm{Na}^{+}$is extruded from the cell by the action of the basolateral $\mathrm{Na}^{+}-\mathrm{K}^{+}-$ ATPase while $\mathrm{Cl}^{!}$is driven from the cell across the apical membrane. In the presence of hypertonicity, the intracellular $\mathrm{Cl}^{\text {! }}$ concentration is increased so any further increase would accentuate its secretion across the apical membrane. This stimulation of $\mathrm{Cl}^{!}$secretion would offset the decrease in $I_{\mathrm{sc}}$ elicited by hypertonic solution. This scenario holds true only if another $\mathrm{Cl}^{!}$-coupled transporter is activated following inhibition of the $\mathrm{Na}^{+}-\mathrm{K}^{+}-2 \mathrm{Cl}^{!}$cotransporter.

Unlike amiloride and bumetanide, the effects of NPPB on hypertonicity-induced $\triangle I_{\mathrm{sc}}$ was osmolyte dependent. In the presence of $\mathrm{NPPB}$, hypertonic $\mathrm{NaCl}$ solution increased rather 
than decreased the $I_{\mathrm{sc}}$. Under basal conditions, NPPB will hyperpolarize the apical membrane; therefore, it will consequently stimulate $\mathrm{Na}^{+}$absorption and increase $I_{\mathrm{sc}}$. The addition of hypertonic $\mathrm{NaCl}$ solution will increase the inward $\mathrm{Na}^{+}$gradient, which, in all likelihood, will potentiate $\mathrm{Na}^{+}$influx when $\mathrm{Cl}^{!}$is elevated inside the cell by NPPB. This enormous inward $\mathrm{Na}^{+}$ flux will increase the $I_{\mathrm{sc}}$ response in such a way that it overwhelms any $I_{\mathrm{sc}}$ decrease evoked by elevated mucosal tonicity. NPPB almost completely abolished the $\triangle I_{\text {sc }}$ induced by hypertonic Dmannitol. Since NPPB did not have the same effect on $\triangle I_{\mathrm{sc}}$ elicited by hypertonic D-mannitol and $\mathrm{NaCl}$, this suggests that role of apical $\mathrm{Cl}^{!}$channels in the epithelial bioelectric response to elevated mucosal tonicity is osmolyte dependent, and at this moment, unclear. The application of hypertonic D-mannitol to the mucosal surface will drive $\mathrm{Na}^{+}$across the apical membrane, decreasing $I_{\mathrm{sc}}$; however, this decrease is almost completely abolished, meaning it must be opposed by an increase in $I_{\mathrm{sc}}$ by some other ion, namely $\mathrm{Cl}^{!}$. The only manner in which this can occur is by movement of $\mathrm{Cl}^{\text {! }}$ out of the cell across the basolateral membrane or through apical $\mathrm{Cl}^{\text {! }}$ channels which are insensitive to NPPB. Exit across the basolateral membrane could occur through either the $\mathrm{Na}^{+}-\mathrm{K}^{+}-2 \mathrm{Cl}^{!}$cotransporter or the $\mathrm{Cl}^{!}-\mathrm{HCO}_{3}{ }^{!}$exchanger and/or possibly the $\mathrm{Na}^{+}$-coupled $\mathrm{Cl}^{!}$transporter if they exist in guinea-pig tracheal epithelium.

The lack of effect of iberiotoxin on $\triangle I_{\text {sc }}$ induced by hypertonic solution suggests that $\mathrm{Ca}^{2+}$-activated $\mathrm{K}^{+}$channels have no apparent role in the epithelial bioelectric response to elevated mucosal tonicity; however, this does not rule out the possibility of other $\mathrm{K}^{+}$channels being involved. Although the effects of ouabain, a Na ${ }^{+}-\mathrm{K}^{+}$-ATPase inhibitor, on $\triangle I_{\mathrm{sc}}$ changes induced by hypertonic solution were examined in this study, the results were inconclusive since ouabain completely abolished the $I_{\text {sc }}$. A further characterization of the possible involvement of the $\mathrm{Na}^{+}-\mathrm{K}^{+}$-ATPase in the epithelial bioelectric response to elevated mucosal tonicity is impossible since its pharmacological inhibitor, ouabain, completely abolishes the baseline $I_{\text {sc }}$.

When comparing the first and second epithelial bioelectric responses to hypertonic $\mathrm{NaCl}$ or D-mannitol solution in the same tissue, the second response to $\mathrm{NaCl}$, not $\mathrm{D}$-mannitol, was significantly less than the first; however, this effect was not as great as the effect of the tested 
inhibitors on reducing $I_{\mathrm{sc}}$. This indicates that the tissue desensitizes in response to hypertonic $\mathrm{NaCl}$ solution and that some of the reduction in $I_{\mathrm{sc}}$ seen with the inhibitors is partially due to desensitization.

The overall goal of this study was to demonstrate that those ion channels involved in hypertonicity-induced smooth muscle relaxation via EpDRF were also involved in the epithelial bioelectric response to elevated tonicity, and if this was found to be the case, this would further support the hypothesis that the synthesis, release, and/or effects of EpDRF are functionally linked to epithelial electrical activity. Fedan et al. (1999) reported that apical $\mathrm{Na}^{+}$and $\mathrm{Cl}^{\prime}$ channels were involved in EpDRF-induced smooth muscle relaxation; however, this study has shown that apical $\mathrm{Na}^{+}$channels and basolateral $\mathrm{Na}^{+}-\mathrm{K}^{+}-2 \mathrm{Cl}^{!}$cotransporters are involved in the epithelial bioelectric response to elevated mucosal tonicity while the role of apical $\mathrm{Cl}^{!}$channels and basolateral $\mathrm{Na}^{+}-\mathrm{K}^{+}-\mathrm{ATPases}$ is unclear.

Because amiloride significantly attenuated hypertonicity-induced smooth muscle relaxation via EpDRF and the epithelial bioelectric response to elevated mucosal tonicity, this provides further evidence that apical $\mathrm{Na}^{+}$channels are intimately involved in the synthesis, release, and/or effects of EpDRF. The role of these channels in mediating EpDRF-induced relaxation responses is unclear. Because the mechanisms by which eukaryotic cells sense increases or decreases in extracellular tonicity are poorly understood (Cohen, 1997), it is possible that these $\mathrm{Na}^{+}$channels are in fact osmotic sensors which initiate EpDRF synthesis and/or signaling by relaying extracellular changes in tonicity to the intracellular environment. If the normal activity of these channels are interfered with by amiloride, it may alter their ability to sense changes in tonicity and/or communicate these changes to the intracellular environment. It is very unlikely that amiloride-sensitive $\mathrm{Na}^{+}$channels are the only, possible osmotic sensors of the airway epithelial cells given the fact that elevations in serosal tonicity also lead to the synthesis, release, and/or effects of EpDRF (Fedan et al., 1999). It is possible that each epithelial surface may have its only osmotic sensor. Because the $\mathrm{Na}^{+}-\mathrm{K}^{+}-2 \mathrm{Cl}^{!}$cotransporter is also 
involved in the epithelial bioelectric response to elevated mucosal tonicity, the possibility exists that this transporter is the osmotic sensor of the basolateral surface.

Fedan et al. (1999) reported that bumetanide produced a modest inhibition $(p<0.07)$ of EpDRF-induced smooth muscle relaxation at added $\mathrm{NaCl}$ concentrations equal or greater than $150 \mathrm{mOsm}$; however, this study has shown that bumetanide attenuated $\triangle \mathrm{I}_{\mathrm{sc}}$ changes evoked by only 120 mOsm of added $\mathrm{NaCl}$ or D-mannitol. The inconsistency between the effects of bumetanide on EpDRF-induced smooth muscle relaxation and hypertonicity-induced $\triangle \mathrm{I}_{\mathrm{sc}}$ suggests that the $\mathrm{Na}^{+}-\mathrm{K}^{+}-2 \mathrm{Cl}^{!}$cotransporter is involved in the epithelial bioelectric response to elevated hypertonicity but not appreciably involved in EpDRF-induced smooth muscle relaxation. From this study, the relationship between the mechanical and bioelectric effects of $\mathrm{Cl}^{!}$channel blockers on hypertonicity-induced smooth muscle relaxation and epithelial bioelectric responses, respectively, cannot be determined. Fedan et al. (1999) used DIDS, a Cl channel blocker and $\mathrm{Cl}^{!}-\mathrm{HCO}_{3}{ }^{!}$exchanger inhibitor (Lamb et al., 2000), rather than NPPB, a

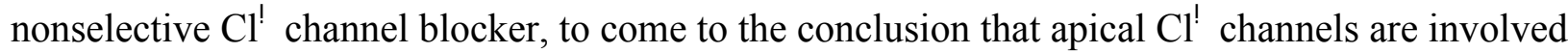
in mediating EpDRF-induced smooth muscle relaxation. The effect seen with DIDS may have nothing to do with its effect upon apical $\mathrm{Cl}^{!}$channels but everything to do with its effect upon the $\mathrm{Cl}^{\prime}-\mathrm{HCO}_{3}{ }^{!}$exchanger. In order for further clarification of the role of $\mathrm{Cl}^{!}$channels and/or transporters, the effect DIDS on $\triangle I_{\text {sc }}$ and NPPB on EpDRF-induced smooth muscle relaxation, respectively, will have to be examined. Finally, there is a discrepancy between the lack of effect of iberiotoxin on the epithelial bioelectric response to elevated mucosal tonicity and its inhibitory effect on the synthesis and/or release of EpDRF. Tamaoki et al. (1997) used iberiotoxin to examine the basal synthesis and/or release of EpDRF, that is, they did not evoke the effects of EpDRF by elevating mucosal or serosal tonicity. For further clarification of the possible involvement of $\mathrm{Ca}^{2+}$-activated $\mathrm{K}^{+}$channels in this response, the effect of iberiotoxin on EpDRFinduced smooth muscle relaxation should be examined by stimulating the effects of EpDRF with hypertonic solution. If iberiotoxin fails to have any effect on EpDRF-induced smooth muscle 
relaxation elicited with hypertonic solution, this could suggest that the basal and stimulated release of EpDRF are controlled by different mechanisms.

The mechanisms by which hypertonicity-induced changes in epithelial bioelectric activity lead to the synthesis, release, and/or effects of EpDRF are unclear; however, the phenomenon of electrical signals releasing autacoids is not unheard of in physiological systems. For example, the release of EpDRF from the airway epithelium, due to altered epithelial electrical activity, may be analogous to the release of ACh from nerve terminals following the propagation of an action potential along the length of a neuron. It is possible that EpDRF, like ACh, is prepackaged in vesicles and released from its cell of origin following a change in the potential difference across the cell membrane.

There were two major points brought about following the conclusion of this study. First, the basic physiological ion transport properties of guinea-pig tracheal epithelium need to be further investigated since this study has shown, contrary to the accepted model, that guinea-pig tracheal epithelium may be a $\mathrm{Cl}^{!}$secretory epithelium rather than a $\mathrm{Na}^{+}$absorbing epithelium. Secondly, these results demonstrate that the epithelial bioelectric response to elevated mucosal tonicity is mediated by the actions of amiloride-sensitive $\mathrm{Na}^{+}$channels and the bumetanidesensitive $\mathrm{Na}^{+}-\mathrm{K}^{+}-2 \mathrm{Cl}^{!}$cotransporter while the role of apical $\mathrm{Cl}^{!}$channels and basolateral $\mathrm{Na}^{+}-$ $\mathrm{K}^{+}$-ATPases is unclear. Because amiloride attenuated hypertonicity-induced smooth muscle relaxation via EpDRF (Fedan et al., 1999) and the epithelial bioelectric response to elevated mucosal tonicity, apical $\mathrm{Na}^{+}$channels appear to be heavily involved in the synthesis, release, and/or effects of EpDRF. 


\section{Lipopolysaccharide-Induced Alterations in Epithelial Electrophysiology in Relation to Epithelium-Derived Relaxing Factor and Airway Reactivity}




\section{Objectives}

Previous work has suggested that the synthesis, release, and/or effects of EpDRF are functionally linked to the electrical activity of epithelial cells (Raeburn and Fedan, 1989; DortchCarnes, et al., 1999; Fedan et al., 1999); therefore, this study sought to determine what effects alterations in epithelial ion transport had upon EpDRF-induced airway smooth muscle relaxation. The hypothesis of this study was that alterations in epithelial ion transport were associated with changes in EpDRF-induced smooth muscle relaxation since the two appear to be functionally linked; therefore, any change in the bioelectric properties of the airway epithelium should subsequently effect the synthesis, release, and/or effects of EpDRF. Because LPS-treatment (4 $\mathrm{mg} / \mathrm{kg}$, i.p.; $18 \mathrm{hr}$ post-treatment) has been shown to alter the basal bioelectric properties of guinea-pig tracheal epithelium (Johnston and Fedan, 1999), this model system was utilized to examine what effect altered epithelial ion transport had upon EpDRF-induced smooth muscle relaxation.

The hypothesis was tested through a variety of different experiments. First, tracheal smooth muscle mechanical responses (contraction and relaxation) and epithelial bioelectric responses $\left(\mathrm{V}_{\mathrm{ms}}\right)$ were measured in isolated, perfused trachea from saline (control)- and LPStreated guinea pigs. If it is found that LPS-treatment alters EpDRF-induced smooth muscle relaxation, it will then be hypothesized that airway reactivity to contractile agonists in vitro and in vivo will also be altered.

\section{Materials and Methods}

\section{Animals}

Specific, pathogen-free, male Dunkin-Hartley guinea pigs (Harlan Sprague Dawley, Inc.; Indianapolis, IN; 405-700 g) were used during the course of this study. The guinea pigs were housed in separate, actively ventilated, micro-isolator cages (Lab Products, Inc.; Seaford, DE) 
within a single room. The room within which these animals were housed were part of an 8800 $\mathrm{ft}^{2}$ modified barrier, animal care facility. Within this facility, the animals were acclimated to their new environment prior to experimentation, given food and water ad libitum, and breathed fresh air. All of the animals in the animal care facility, as well as the facility itself, were under the supervision of a full-time veterinarian.

Throughout this study, the guinea pigs were sacrificed according to a standard protocol. The animals were first anesthetized intraperitoneally (i.p.) with sodium pentobarbital (Veterinary Laboratories, Inc.; Lenexa, $\mathrm{KS} ; 65 \mathrm{mg} / \mathrm{kg}$ ), and once it was determined that the animals were unresponsive to any stimuli, they were exsanguinated by severing the abdominal aorta and cutting the heart.

All of the experimental protocols in this study were approved by the Institutional Animal Care and Use Committee (IACUC) while the facilities where the experimental protocols were conducted were fully accredited by the Association for the Assessment and Accreditation of Laboratory Animal Care (AAALAC) International. These facilities have been accredited by AAALAC International since February 18, 1987.

\section{In Vivo Saline- and Lipopolysaccharide-Treatments}

Guinea pigs were injected i.p. with $4 \mathrm{mg} / \mathrm{kg}$ lipopolysaccharide (LPS) from Salmonella typhimurium or a volume equivalent of $0.9 \%$ sodium chloride irrigation, USP (saline, control; Baxter Healthcare Corporation; Deerfield, IL). Eighteen hr after treatment, in vitro or in vivo experiments were conducted.

\section{Isolated, Perfused Trachea Apparatus}

The isolated, perfused trachea apparatus (Figure 21) is a unique in vitro preparation which is used to measure tracheal smooth muscle contractile and relaxant responses in response to various pharmacological agents (Munakata et al., 1988; Fedan and Frazer, 1992). 
This preparation consisted of two separate, water-jacketed organ baths whose temperature was maintained at $37^{\circ} \mathrm{C}$. The $30 \mathrm{ml}$ extraluminal (EL) or serosal bath and the $30 \mathrm{ml}$ intraluminal (IL) or mucosal bath were filled with MKH solution that was continuously gassed with $95 \% \mathrm{O}_{2}-5 \% \mathrm{CO}_{2}$. In order to remove the trachea from the guinea pig, the animal was sacrificed as previously described. The trachea was exposed in vivo, a $4.2 \mathrm{~cm}$ segment excised, placed in $\mathrm{MKH}$ solution gassed with $95 \% \mathrm{O}_{2}-5 \% \mathrm{CO}_{2}$, and cleaned by removing extraneous tissue. The trachea was then mounted on a stainless steel perfusion holder, stretched to its in situ length, and placed into the EL bath containing gassed MKH solution. After attaching the necessary tubing, the trachea was perfused, at a constant rate of $34 \mathrm{ml} / \mathrm{min}$, with gassed, recirculating $\mathrm{MKH}$ solution $\left(37^{\circ} \mathrm{C}\right)$ from the separate IL bath. Since the tracheal smooth muscle and epithelium were in direct contact with the gassed MKH solution in the EL and IL baths, respectively, pharmacological agents added to the EL bath have direct access to the smooth muscle while those agents added to the IL bath must first diffuse through the epithelium before reaching the smooth muscle. This preparation, therefore, allowed one to study the degree to which the epithelium can modulate airway reactivity. 


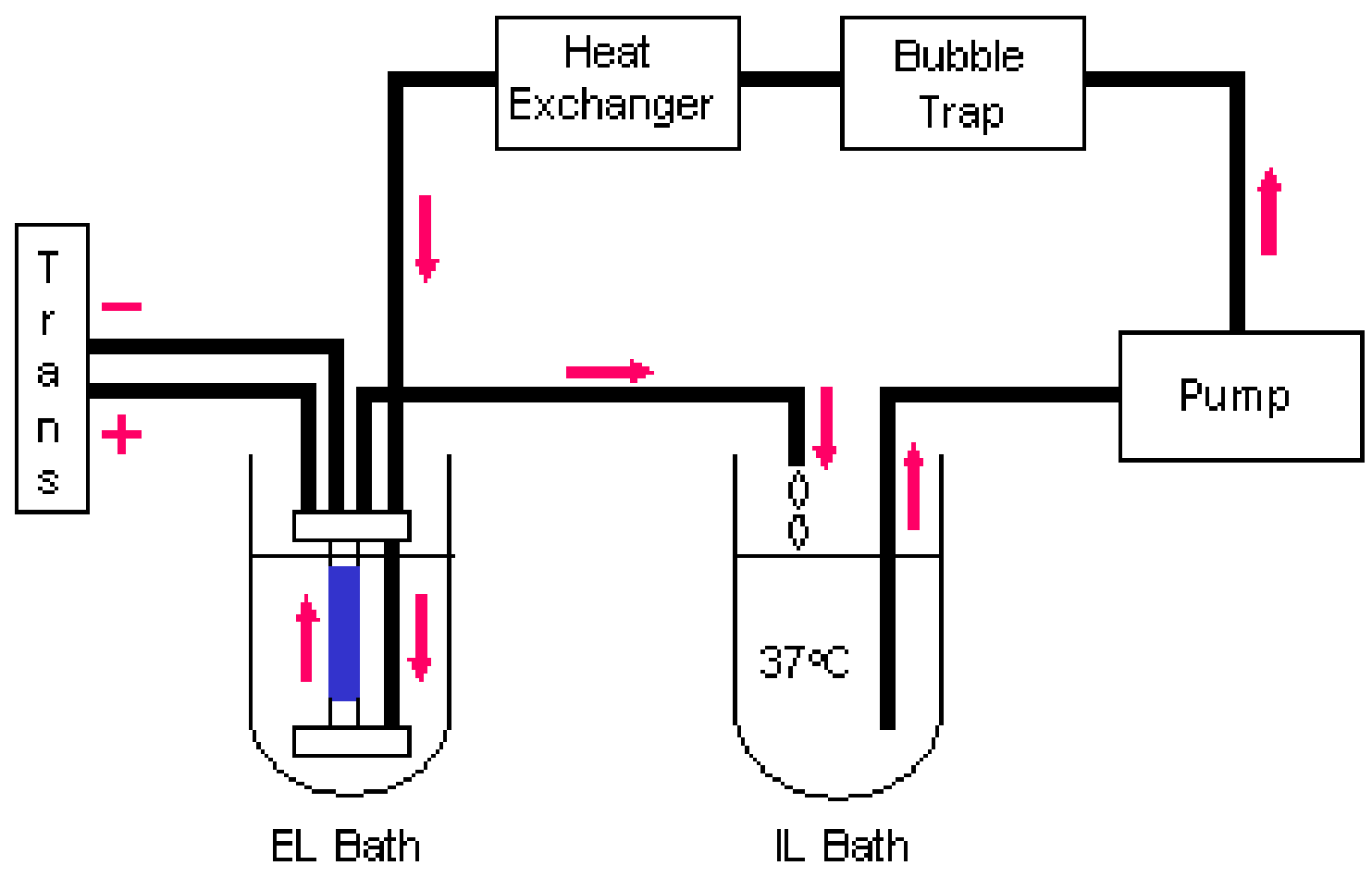

Figure 21. Isolated, perfused trachea apparatus. A $4.2 \mathrm{~cm}$ length of guinea-pig trachea, shownin blue, is attached to a perfusion holder and placed in the extraluminal (EL) or serosal bath containing modified Krebs-Henseleit $(\mathrm{MKH})$ solution maintained at $37^{\circ} \mathrm{C}$. Once all of the necessary tubing is attached, the intraluminal (IL) or mucosal surface of the trachea is perfused at a constant rate with recirculating $\mathrm{MKH}$ solution also maintained at $37^{\circ} \mathrm{C}$. The red arrows indicate the direction of fluid flow through the preparation. As explained in the text, this preparation can measure contractile and relaxant responses of the tracheal smooth muscle (illustration produced by and courtesy of Jeffrey S. Fedan, Ph.D.). 
Contractile and relaxant responses of the tracheal smooth muscle were measured as changes in the inlet minus outlet pressure difference $\left(\triangle \mathrm{P} ; \mathrm{cm} \mathrm{H}_{2} \mathrm{O}\right)$ between the side holes of two indwelling catheters which became inserted into the lumen upon mounting the trachea to the holder (Figure 22). The proximal and distal ends of the trachea were attached with suture (Deknatel, Inc.; Fall River, MA) around the inlet and outlet catheters, respectively. The inlet and outlet catheters were connected by pressure lines to the positive and negative sides, respectively, of a differential pressure transducer (Validyne Engineering Corp.; Northridge, CA). Because the rate of fluid movement through the trachea was kept constant $(34 \mathrm{ml} / \mathrm{min})$, the $\triangle \mathrm{P}$ responses were representative of changes in tracheal cross-sectional area associated with contraction and relaxation; therefore, an increase or decrease in $\triangle \mathrm{P}$ was associated with smooth muscle contraction and relaxation, respectively (Munakata et al., 1989). These responses were recorded on a conventional strip chart recorder (Gould Instrument Systems; Valley View, OH). In all instances, the transmural pressure was zeroed, and the preparations were equilibrated for $3 \mathrm{hr}$ before any experimental manipulations were initiated. During the equilibration period, however, the MKH solution in both baths was changed every $15 \mathrm{~min}$. 


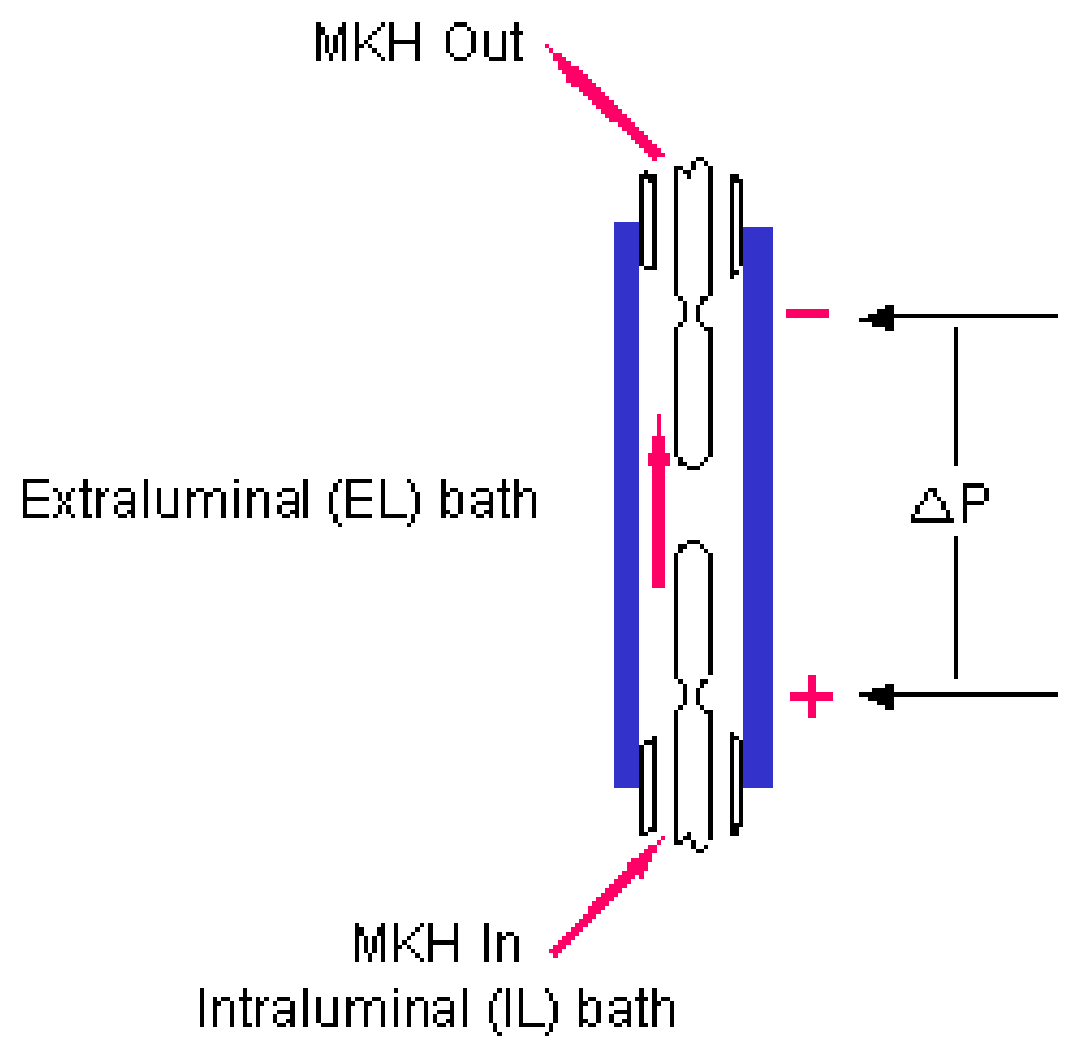

Figure 22. Differential pressure measurement in the isolated, perfused trachea apparatus. Contractile and relaxant responses of the tracheal smooth muscle are measured as changes in the inlet minus outlet pressure difference $(\triangle \mathrm{P})$ between the side holes of the indwelling perfusion holder catheters. The trachea is shown in blue, and the red arrows indicate the direction of fluid flow through the trachea (illustration produced by and courtesy of Jeffrey S. Fedan, Ph.D.). 


\section{Modified Isolated, Perfused Trachea Apparatus}

Recent modifications to the isolated, perfused trachea apparatus now permit transepithelial potential difference $\left(\mathrm{V}_{\mathrm{ms}}\right.$; Dortch-Carnes et al., 1999) to be measured simultaneously with smooth muscle contractile and relaxant responses at the proximal and distal ends of the trachea. The experimental setup of this preparation was unchanged from before (Figure 21); however, modifications of the perfusion holder were made to measure epithelial bioelectric responses. Because epithelial bioelectric responses were measured with this preparation, a plastic perfusion holder, rather than a stainless steel one, was used. For the most part, the plastic perfusion holder did not affect the manner in which smooth muscle mechanical responses $(\triangle \mathrm{P})$ were measured; however, the flow rate in this preparation was kept constant at $22 \mathrm{ml} / \mathrm{min}$ instead of the $34 \mathrm{ml} / \mathrm{min}$ used with the stainless steel holder, which permitted transmural pressure to be zeroed in the face of a greater fluid resistance of the plastic holder. A schematic illustration of the modified perfusion holder is shown in Figure 23.

During the course of this study, $\mathrm{V}_{\mathrm{ms}}$ measurements were made at the proximal end of the trachea, and the epithelium surrounding the opening of the inlet catheter was the point source for the measurement of the $\mathrm{V}_{\mathrm{ms}}$. Two silver/silver chloride calomel half cells, one designated as IL or mucosal $\left(\mathrm{V}_{\mathrm{m}}\right)$ and the other as EL or serosal $\left(\mathrm{V}_{\mathrm{s}}\right)$, were used to measure the $\mathrm{V}_{\mathrm{ms}}$. Each calomel half cell was in contact with the fluid bathing either the EL or IL surfaces of the trachea by means of Ussing voltage electrode cartridges [World Precision Instruments (WPI); Sarasota, FL] filled with $3 \mathrm{M} \mathrm{KCl}$ in $4 \%$ melted bacteriological agar. The $\mathrm{V}_{\mathrm{ms}}$ was equal to the sum of $\mathrm{V}_{\mathrm{m}}, \mathrm{V}_{\mathrm{s}}$, and an offset potential. Before the trachea was mounted on the perfusion holder, any potential difference between the electrodes, not exceeding $\pm 2 \mathrm{mV}$, was dialed to zero (Dortch-Carnes et al., 1999). 


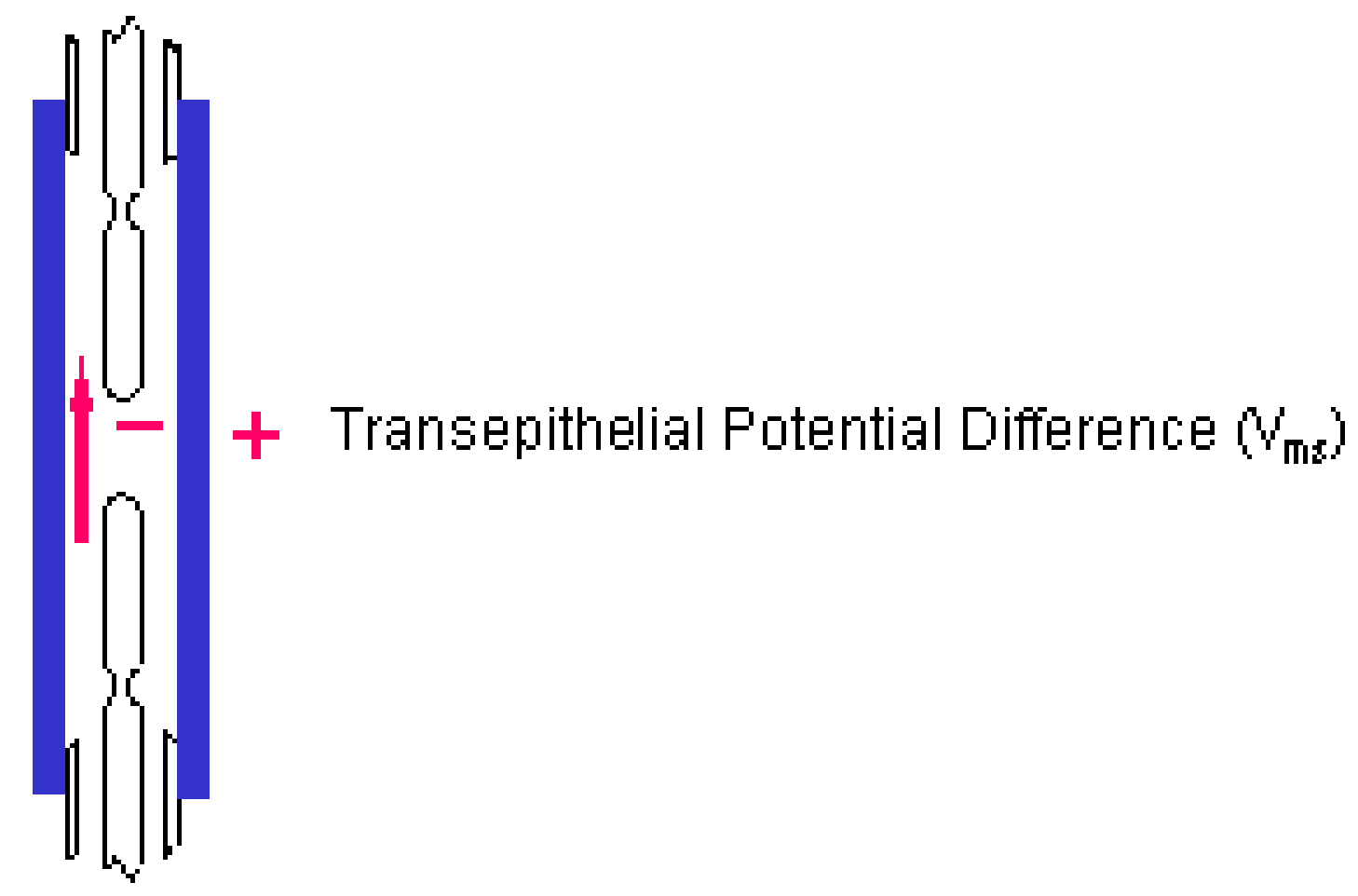

Figure 23. $\mathrm{V}_{\mathrm{ms}}$ measurement in the modified isolated, perfused trachea apparatus. The $\mathrm{V}_{\mathrm{ms}}$ can be measured at either the proximal or distal end of the trachea through the side hole of the indwelling perfusion holder catheter; however, this study measured the $\mathrm{V}_{\mathrm{ms}}$ only at the proximal end of the trachea. Again, the trachea is shown in blue and the red arrows indicate the direction of fluid flow through the trachea. The negative sign (! ) refers to the fact that the airway epithelium generates a lumen-negative potential with respect to the positive $(+)$, basolateral surface (illustration produced by and courtesy of Jeffrey S. Fedan, Ph.D.). 
A two-channel DVC-1000 voltage/current clamp (WPI; Sarasota, FL) was used in the measurement of tracheal $\mathrm{V}_{\mathrm{ms}}$ responses. The $\mathrm{V}_{\mathrm{ms}}$ was recorded continuously under open-circuit conditions on a conventional strip chart recorder (Gould Instrument Systems; Valley View, OH).

\section{In Vitro Elevated Tonicity Concentration-Response Curves}

EpDRF release was stimulated by increasing the tonicity of the MKH solution perfusing the IL surface of the trachea with either $\mathrm{NaCl}, \mathrm{KCl}$, D-mannitol, or urea. After obtaining a stable contraction with extraluminally-added $\mathrm{MCh}\left(3 \times 10^{! 7} \mathrm{M} ; \sim \mathrm{EC}_{50}\right.$ for contraction), concentrationresponse curves for relaxation were generated by adding the osmolytes in stepwise-increasing, cumulative concentrations to the IL bath. Equiosmolar, rather than equimolar, concentrations of the osmolytes were added to the IL bath. The relaxation responses were normalized as a percentage of the MCh-induced contraction.

In addition, concentration-response curves for $\triangle \mathrm{V}_{\mathrm{ms}}$ were generated by adding $\mathrm{NaCl}$ in stepwise-increasing, cumulative concentrations to the EL and/or IL baths in the presence and absence of MCh. The EL and IL curves were obtained from each trachea, the IL curve first, followed 90 min later by the EL curve.

\section{In Vitro Effects of Agents on EpDRF-Induced Smooth Muscle Relaxation}

During the course of this study, inhibitors of enzymes important to the synthesis of nitric oxide and prostanoids were utilized to determine whether or not they affected EpDRF-induced smooth muscle relaxation. Each inhibitor was added to the EL and IL baths 30 min prior to inducing muscle tone with extraluminally-added $\mathrm{MCh}\left(3 \times 10^{! 7} \mathrm{M} ; \sim \mathrm{EC}_{50}\right)$. When relaxation concentration-response curves were generated, the effect of an inhibitor upon responses was compared to the responses in control preparations where the inhibitor was absent. 


\section{In Vitro Effects of Agents on the Basal $\mathrm{V}_{\mathrm{ms}}$}

In some experiments, the effects of indomethacin, $\mathrm{N}^{\top}$-nitro-L-arginine methyl ester (LNAME), atropine, sodium nitroprusside, and amiloride on the basal $\mathrm{V}_{\mathrm{ms}}$ were determined. Indomethacin, L-NAME, atropine, and sodium nitroprusside were added to the EL and IL baths simultaneously while amiloride was only added to the IL bath.

The effect of LPS on the basal $V_{m s}$ was determined in tracheas from naive animals by adding $10 \mu \mathrm{g} / \mathrm{ml}$ of LPS to the EL and IL baths simultaneously. An untreated, control preparation was run in parallel as a time control.

\section{In Vitro MCh Concentration-Response Curves}

MCh concentration-response curves were generated by adding MCh in stepwiseincreasing, cumulative concentrations to the EL and IL baths. EL and IL MCh concentrationresponse curves were obtained from each trachea, the EL curve first, followed 90min later by the IL curve, with washes every $15 \mathrm{~min}$. The contractile responses to $\mathrm{MCh}$ were normalized as a percentage of the maximum MCh-induced contraction.

\section{Tracheal Epithelium Removal}

In isolated, perfused trachea experiments requiring removal of the tracheal epithelium, a trimmed, 6-cm segment of pipe cleaner (Fisher Scientific; Pittsburgh, PA) was slowly and carefully inserted into the tracheal lumen and then withdrawn while rotating slowly (Fedan and Frazer, 1992).

\section{Pulmonary Function Measurements}

A plethysmograph is used to measure lung volumes and pulmonary function parameters in vivo. In this study, a two-chambered, whole-body plethysmograph (Figure 24) was used (Buxco Electronics, Inc.; Sharon, CT). 
A conscious guinea pig was placed in the plethysmograph so that its head and thorax were in the head and body chamber, respectively. In addition, two latex collars were placed around the animal's neck in order to restrain it. Besides restraining the animal, the collars form an airtight seal between the two chambers. The guinea pig breathed fresh air through a port in the head chamber, and administered agents entered the head chamber through a separate, aerosol port. The head and thoracic chambers were equipped with identical wire screen pneumotachographs and SEN1 differential pressure transducers. The differential pressure transducers measured pressure changes within each chamber and were connected to an LS-20 noninvasive airway mechanics analyzer. The results were then logged and displayed on a computer screen. 


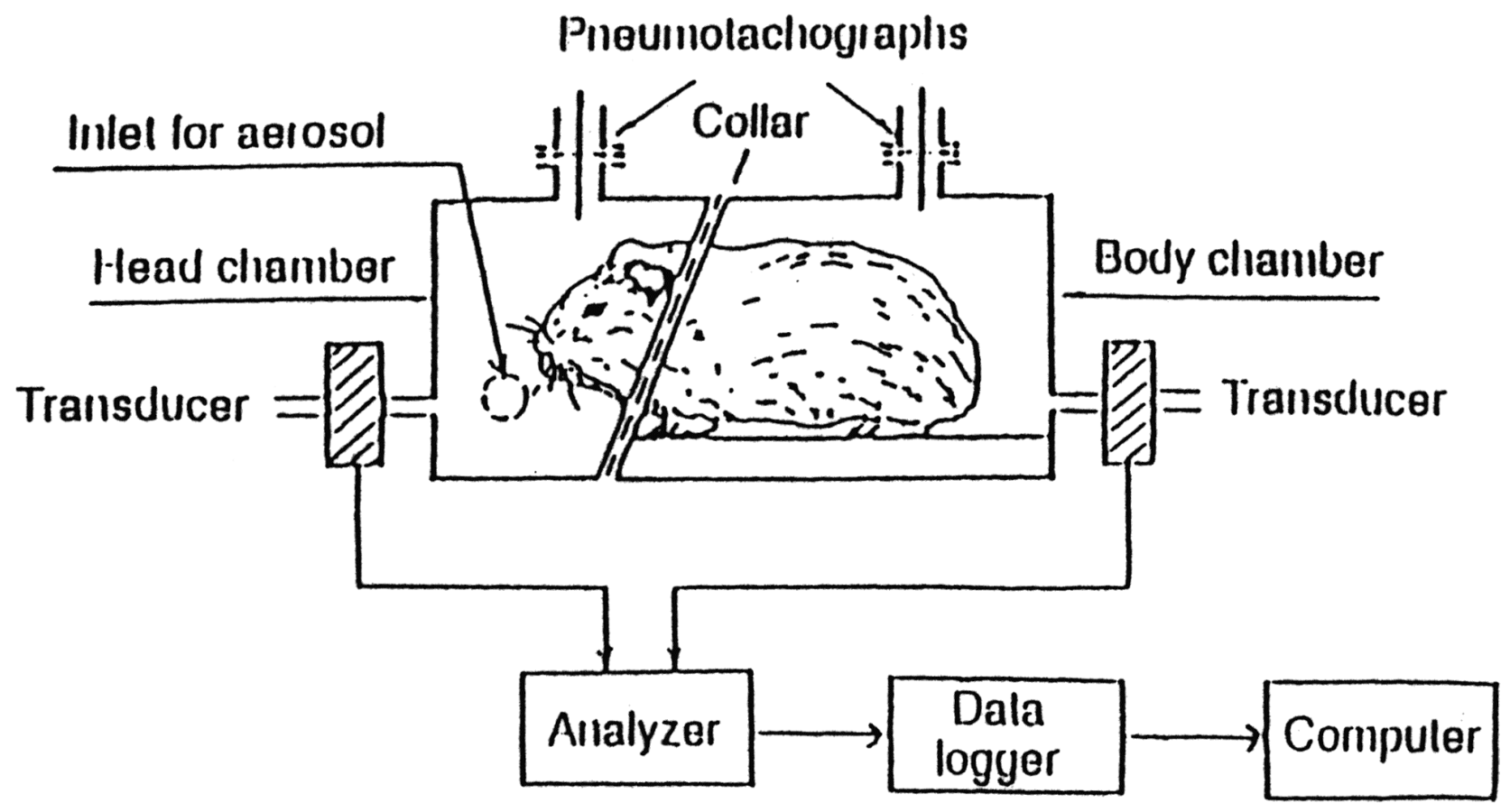

Figure 24. Two-chambered, whole-body plethysmograph. The two-chambered, whole-body plethysmograph is used along with its associated equipment to measure in vivo pulmonary function parameters and reactivity to inhaled, MCh aerosol. Reprinted from $J$ Pharmacol Toxicol Methods, Chong BTY, Agrawal DK, Romero FA and Townley RG, Measurement of bronchoconstriction using whole-body plethysmograph: Comparison of freely moving versus restrained guinea pigs, pp 163-168, 1998, with permission from Elsevier Science. 
The plethysmograph was capable of measuring ten pulmonary function parameters from a single breath, but this study was only concerned with two: breathing frequency (BF) and specific airway resistance (SRaw). The first parameter of interest, BF, was expressed as the number of breaths per minute (BPM). The computer analyzes each breath with regard to four exclusion criteria: (1) the minimum inspiratory time, (2) the maximum inspiratory time, (3) the peak expiratory flow, and (4) the percent maximum deviation between the inspiratory and expiratory volume. A breath must fall in the normal range of all four criteria in order to be recorded (Lomask, 1996).

SRaw is the product of airway resistance and thoracic gas volume and is expressed in cm $\mathrm{H}_{2} \mathrm{O} \bullet \mathrm{s}$. SRaw is an indicator of airway resistance, and in mathematical terms, it can be described by the following equation:

$$
\mathrm{SRaw}=\tan 2\left(\mathrm{P}_{\mathrm{atm}}-47\right)(1.36)\left(\mathrm{T}_{\mathrm{i}} / \mathrm{B}\right)
$$

Figure 25 illustrates the derivation of theta (2) which can also be expressed mathematically by the following equation:

$$
2=\mathrm{B}(\triangle \mathrm{T}) / \mathrm{T}_{\mathrm{i}}
$$

$\triangle \mathrm{T}$ is the delay time between the zero crossings of nasal and thoracic flows at the end of inspiration. $\triangle \mathrm{T}$ is computed in seconds and used to calculate 2, which is the phase shift between the nasal and thoracic flows. The additional components of the SRaw equation are as follows: (1) 1.36 is the conversion factor from $\mathrm{mm} \mathrm{Hg}$ to $\mathrm{cm}_{2} \mathrm{O}$, (2) $\mathrm{P}_{\mathrm{atm}}$ is the barometric pressure in $\mathrm{mm} \mathrm{Hg}$, and (3) $\mathrm{T}_{\mathrm{i}}$ is the inspiratory time (Lomask, 1996).

Both SRaw and BF data were logged at 6 sec intervals with the mean of ten consecutive averages taken as the measurement at each time point. 


\section{In Vivo MCh Dose-Response Curves}

The guinea pig was placed in the two-chambered, whole-body plethysmograph $24 \mathrm{hr}$ prior to generating an in vivo $\mathrm{MCh}$ concentration-response curve, and the animal was acclimated to the chamber for $2 \mathrm{hr}$ in air. This reduced the stress that the animal experienced when placed in the plethysmograph a second time. On the second day, the animal was again placed in the plethysmograph and acclimated in air for $20 \mathrm{~min}$. During this $20 \mathrm{~min}$ period, SRaw and BF were continuously recorded to get basal SRaw and BF values. Next, a saline aerosol vehicle was delivered to the head chamber for $3 \mathrm{~min}$, and at the end of this period, SRaw and BF data were taken at $6 \mathrm{sec}$ intervals for $3 \mathrm{~min}$ as the animl breathed air. After a $30 \mathrm{~min}$ resting period, the lowest concentration of $\mathrm{MCh}$ aerosol was delivered to the head chamber for $3 \mathrm{~min}$. Once the aerosol delivery was stopped, SRaw and BF were again measured for 3 min as the animal breathed air. After $30 \mathrm{~min}$, SRaw and BF returned to their basal values, and the next higher concentration of $\mathrm{MCh}$ was delivered to the chamber and the procedure was repeated with increasing MCh concentrations. Unlike in vitro experiments, a full in vivo dose-response curve was impossible to generate because the animals cannot tolerate dyspnea accompanying a maximum response; therefore, $\mathrm{ED}_{50}$ 's and maximum responses could not be determined. $\mathrm{MCh}$ concentrations ranging between 0.0316 and $3.16 \mathrm{mg} / \mathrm{ml}$ were delivered. 


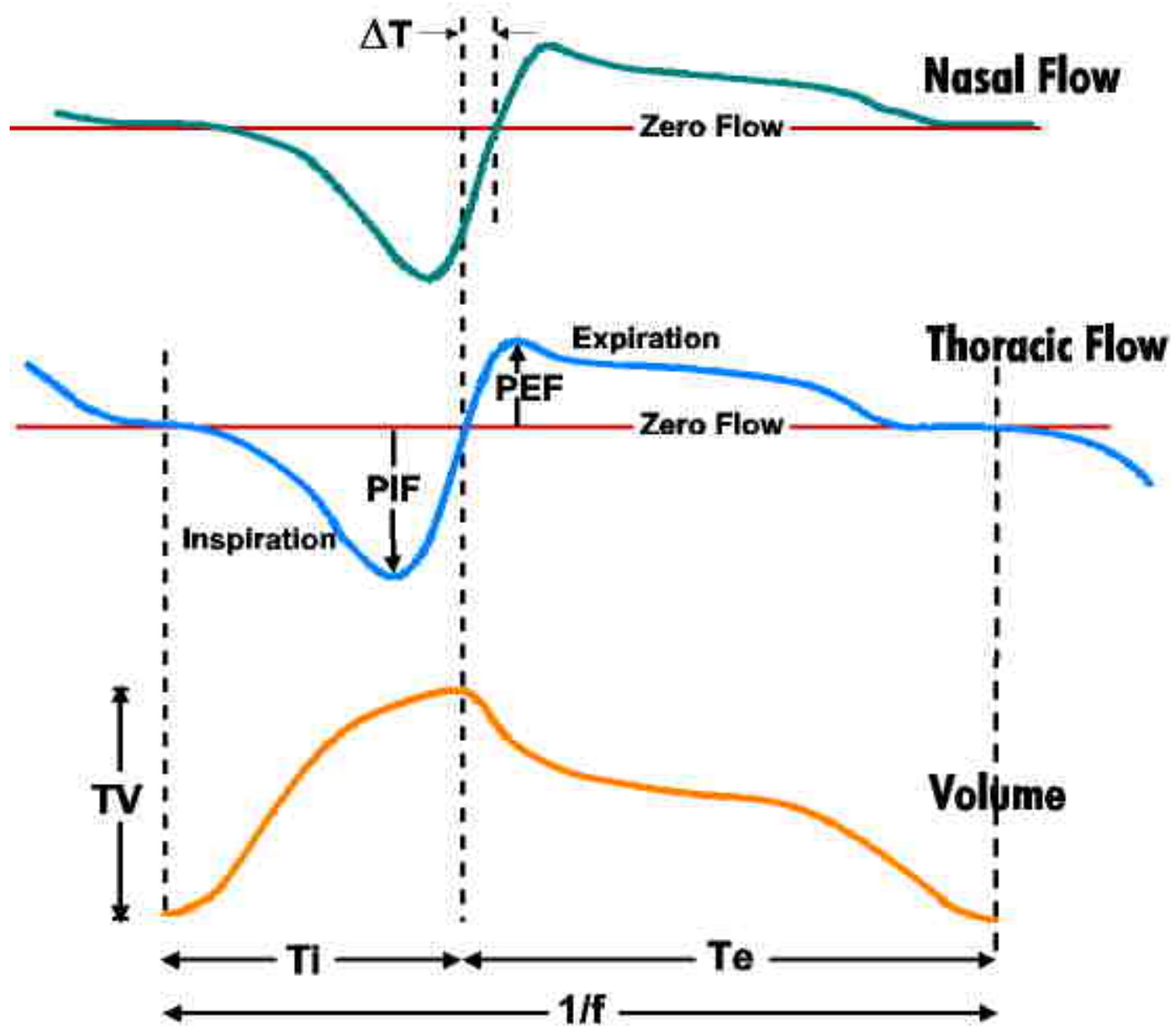

Figure 25. Definition of theta. A schematic illustration of guinea-pig head (nasal) and body (thoracic) airflow during inspiration and expiration as recorded by the data collection system associated with the two-chambered, whole-body plethysmograph. Since thoracic airflow precedes nasal airflow at the zero crossing following inspiration, $\triangle \mathrm{T}$ (delay time) can be calculated, which is subsequently used to calculate theta (2). Reprinted from Lomask K (1996) BioSystem XA User's Manual, Version 0.9.3, Buxco Electronics, Inc., Sharon, CT with permission from Buxco Electronics, Inc. 
The $\mathrm{MCh} \mathrm{PC}_{200}$, the provocative $\mathrm{MCh}$ concentration producing a two-fold increase in basal SRaw, was calculated by linear interpolation of graphed data. All aerosols were generated using an Ultra Neb 99 DeVilbiss nebulizer (DeVilbiss Co.; Somerset, PA) containing $5 \mathrm{ml}$ of solution.

When examining the effects of saline- and LPS-treatments on airway reactivity to inhaled MCh aerosol, a standard protocol was followed. Naive animals were acclimated on day 1 and the same animals exposed to MCh aerosols on day 2. These animals were then treated with saline or LPS, and $18 \mathrm{hr}$ post-treatment, airway reactivity to inhaled MCh was determined. In all instances, each animal served as its own control since reactivity to MCh was determined before and after saline- and LPS-treatments.

\section{In Vivo Administration of L-NAME}

To test the possibility that inhaled L-NAME aerosol may affect airway reactivity to inhaled MCh in saline- and LPS-treated animals, guinea pigs were exposed to L-NAME aerosol $(1.2 \mathrm{mM})$ for $5 \mathrm{~min}$ prior to the delivery of each MCh concentration. Two MCh concentrationresponse curves were generated in both saline- and LPS-treated animals, the first in the absence and the second after treatment with L-NAME.

\section{Histological Examination of Guinea-Pig Airways}

Following anesthesia and exsanguination, trachea and lungs from saline- and LPS-treated animals were inflated with $15-20 \mathrm{ml}$ of $10 \%$ buffered formalin phosphate (Fisher Scientific; Pittsburgh, PA) and removed en bloc. Sections of the trachea as well as the azygous, left, right apical, right cardiac, and right diaphragmatic lobes of the lung were then embedded with paraplast-plus paraffin before being cut into $5 \mu \mathrm{m}$ slices with a microtome. Individual sections were then placed on glass microscope slides where they were stained according to Harris' hematoxylin and eosin procedure. Following the conclusion of section staining, section mounting 
was completed by placing a cover slip over the tissue section. Finally, the slides were examined in a "blind" fashion under the light microscope by a veterinary pathologist.

\section{Solutions and Reagents}

The MKH solution contained (mM): $\mathrm{NaCl}$ (113.0), $\mathrm{KCl}(4.8), \mathrm{CaCl}_{2}$ (2.5), $\mathrm{KH}_{2} \mathrm{PO}_{4}$ (1.2), $\mathrm{MgSO}_{4}$ (1.2), $\mathrm{NaHCO}_{3}$ (25.0), and glucose (5.7). The MKH solution was continuously gassed with $95 \% \mathrm{O}_{2}-5 \% \mathrm{CO}_{2}$ and maintained at $37^{\circ} \mathrm{C}$ and a $\mathrm{pH}$ of 7.4 . In addition, the osmolarity of the MKH solution was $281.2 \pm 0.6 \mathrm{mOsm}(n=5$; Osmette A Automatic Osmometer, Precision Systems, Inc.; Sudbury, MA).

All chemical reagents used during the course of this study were purchased from Sigma Chemical Co. (St. Louis, MO) and dissolved in saline unless otherwise noted. Indomethacin was prepared in $100 \mathrm{mM}$ sodium carbonate.

\section{Analysis of Results}

The data obtained from in vitro concentration-response curves examining EpDRFinduced smooth muscle relaxation were normalized as a percent of the MCh-induced contraction while in vitro $\triangle \mathrm{P} M C h$ concentration-response curves were normalized as a percent of the maximum response. Data normalization was necessary in these instances to avoid large variability among $\triangle \mathrm{P}$ values (Fedan and Frazer, 1992). Since $\triangle \mathrm{P}$ is dependent upon the fifth power of the radius of the trachea, small differences in the internal diameter of the trachea can lead to large variability among $\triangle \mathrm{P}$ values (Munakata et al., 1989). Geometric $\mathrm{EC}_{50}$ values were derived from the least squares analysis of a sigmoidal curve fit (Sigma Plot ${ }^{\circledR}$ ). When comparing $\mathrm{EC}_{50}$ values statistically, normally-distributed $\mathrm{EC}_{50}$ values were used. The $\mathrm{EC}_{50}$ 's and maximum responses were then analyzed using either the paired or nonpaired Student's $t$ test, the MannWhitney rank sum test, or one-way repeated measures analysis of variance (ANOVA), as appropriate. For concentration-response curves examining $\triangle \mathrm{V}_{\mathrm{ms}}$, the $\mathrm{EC}_{50}$ 's and maximum 
responses were derived and statistically compared in the same manner as described for the $\triangle \mathrm{P}$ responses.

When examining the effect of agents on the $\mathrm{V}_{\mathrm{ms}}$, the data were statistically analyzed using either the paired or nonpaired Student's $t$ test or the Mann-Whitney rank sum test, as appropriate.

For in vivo airway reactivity studies, pre-treatment and post-treatment BF, SRaw, and MCh $\mathrm{PC}_{200}$ values among saline- and LPS-treated animals were statistically compared using the paired or nonpaired Student's $t$ test or Mann-Whitney rank sum test, as appropriate. The effect of L-NAME on $\mathrm{MCh} \mathrm{PC}_{200}$ values among saline- and LPS-treated animals was compared using the paired or nonpaired Student's $t$ test, as appropriate.

All of the results, except where noted, were expressed as the mean \pm S.E.M. (standard error of the mean) where $n$ is the number of separate experiments. In every experiment, $p<0.05$ was considered significant. 


\section{Results}

\section{Effect of LPS-treatment on basal $V_{m s}$ in isolated, perfused trachea}

LPS-treatment increased the basal $\mathrm{V}_{\mathrm{ms}}$ in isolated, perfused trachea. The basal $\mathrm{V}_{\mathrm{ms}}$ was ! 14.6 $\pm 1.2 \mathrm{mV}$ and ! $28.3 \pm 1.9 \mathrm{mV}$ for the saline- and LPS-treated groups, respectively (Figure 26).

\section{Effect of LPS-treatment on EpDRF-induced smooth muscle relaxation in isolated, perfused trachea}

In order to test the hypothesis that LPS-induced alterations in epithelial ion transport were associated with perturbations in EpDRF-induced smooth muscle relaxation, smooth muscle reactivity to EpDRF was measured in isolated, perfused tracheas from saline- and LPS-treated guinea pigs. EpDRF-induced smooth muscle relaxation was stimulated by increasing the tonicity of the IL MKH solution with four different osmolytes ( $\mathrm{NaCl}$, D-mannitol, urea, and $\mathrm{KCl}$ ) following contraction of the smooth muscle with extraluminally-applied MCh $\left(3 \times 10^{! 7} \mathrm{M}\right.$; $\sim \mathrm{EC}_{50}$ ). Figures 27-29 and Table 1 summarize the results of this study. When $\mathrm{NaCl}$ and Dmannitol were used to increase the tonicity of the IL bath, EpDRF-induced smooth muscle relaxation was potentiated in tracheas from LPS-treated animals (Figure 27). When urea was used, there was no difference in the maximum relaxation responses between the two treatment groups; however, there was a small, but significant decrease in smooth muscle reactivity to EpDRF in the LPS-treated group, as demonstrated by a rightward shift of the concentrationresponse curve (Figure 28). 


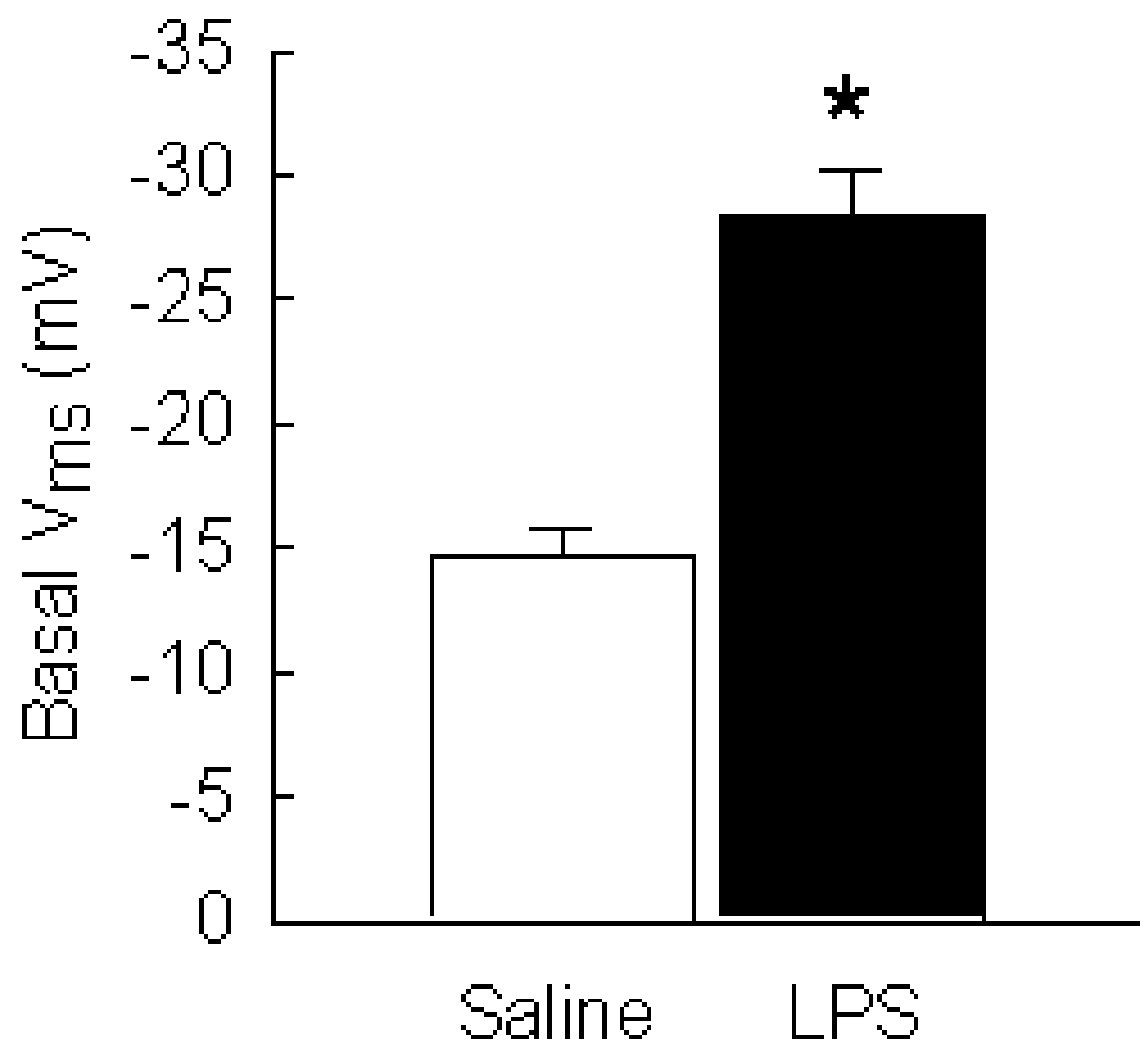

Figure 26. Effect of LPS-treatment on the basal $V_{m s}$ in isolated, perfused trachea. Basal $V_{m s}$ values were obtained from tracheas removed from saline- and LPS-treated animals $18 \mathrm{hr}$ posttreatment. Saline- and LPS-treated, $n=20 .{ }^{*} p<0.05$ compared to saline as determined by a nonpaired Student's $t$ test. 

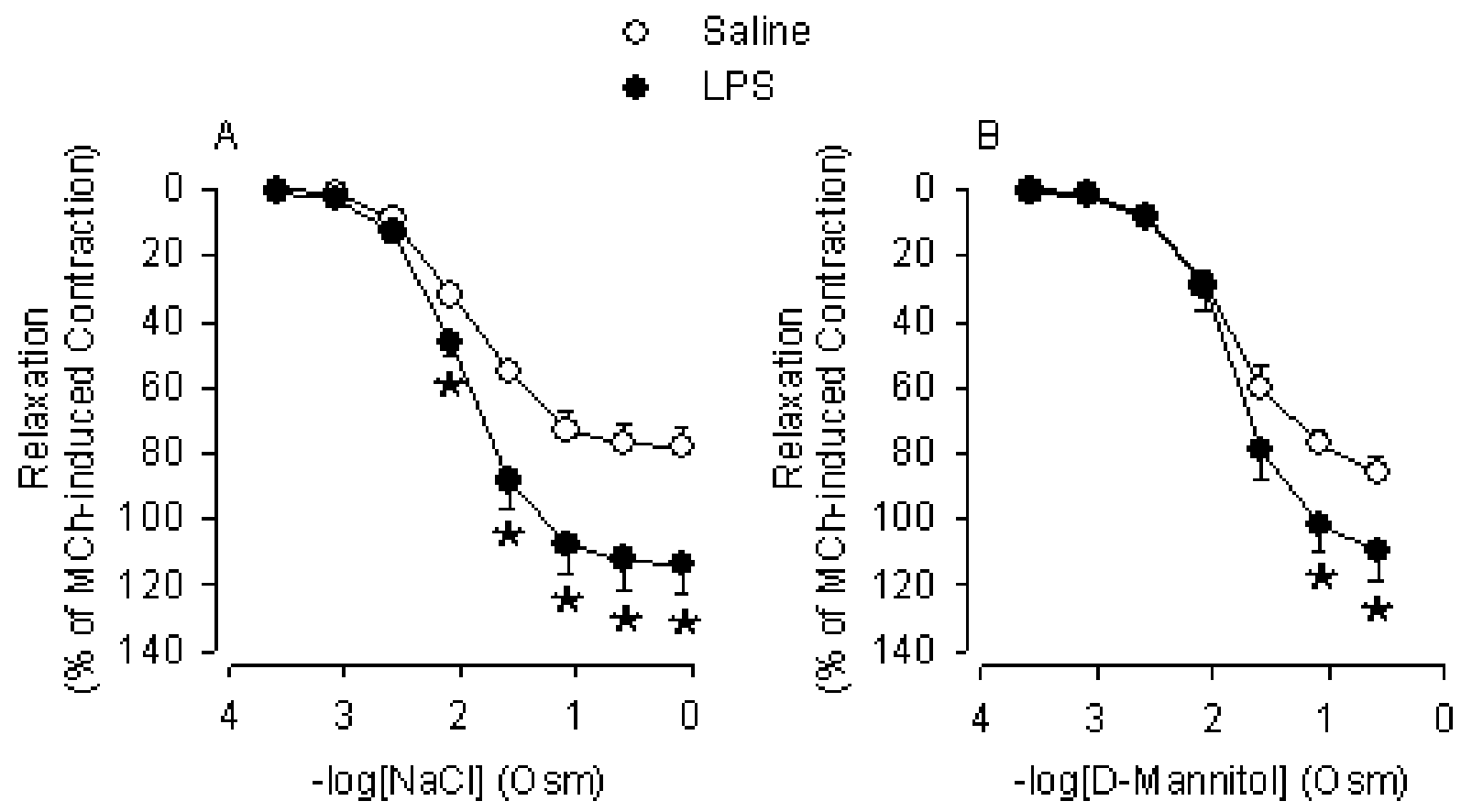

Figure 27. Effect of LPS-treatment on EpDRF-induced smooth muscle relaxation elicited with IL MKH solution made hypertonic with added (A) $\mathrm{NaCl}$ or (B) D-mannitol. The curves were obtained from tracheas removed from saline- and LPS-treated animals $18 \mathrm{hr}$ post-treatment. The preparations were contracted with extraluminally-applied $\mathrm{MCh}\left(3 \times 10^{!} 7 \mathrm{M} ; \sim \mathrm{EC}_{50}\right)$ before $\mathrm{NaCl}$ or D-mannitol were added in stepwise-increasing, cumulative concentrations to the IL bath. EpDRF-induced smooth muscle relaxation was potentiated in tracheas from LPS-treated animals when $\mathrm{NaCl}$ or D-mannitol were used to evoke EpDRF release. The osmolyte concentrations shown in the abscissa of the figures refer to the osmolar concentrations of osmolyte added to the IL MKH solution. Equiosmolar, rather than equimolar, concentrations of $\mathrm{NaCl}$ and D-mannitol were added to the IL bath. For $\mathrm{NaCl}$ the osmolarity is twice the molarity and for D-mannitol the osmolarity is the same as the molarity. Saline- and LPS-treated, $n=8$ for $\mathrm{NaCl}$ and $n=6$ for Dmannitol. ${ }^{*} p<0.05$ compared to saline as determined by a nonpaired Student's $t$ test. 

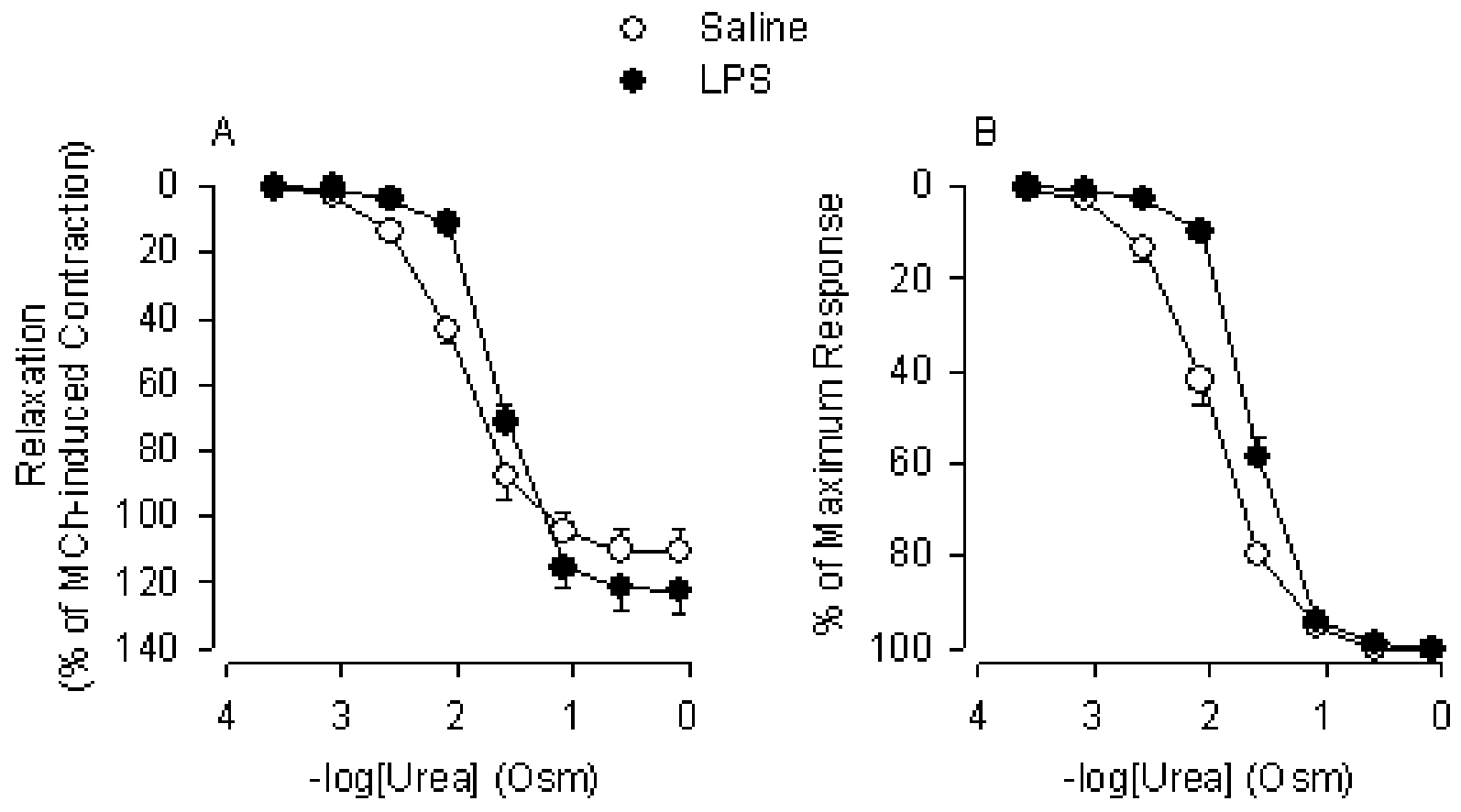

Figure 28. Effect of LPS-treatment on EpDRF-induced smooth muscle relaxation elicited with IL MKH solution made hypertonic with added urea. The curves were obtained from tracheas removed from saline- and LPS-treated animals $18 \mathrm{hr}$ post-treatment. The preparations were contracted with extraluminally-applied $\mathrm{MCh}\left(3 \times 10^{! 7} \mathrm{M} ; \sim \mathrm{EC}_{50}\right)$ before urea was added in stepwise-increasing, cumulative concentrations to the IL bath. LPS-treatment did not affect the maximum relaxation response; however, it caused a small, but significant decrease in smooth muscle reactivity to EpDRF, as demonstrated by an increase in the $\mathrm{EC}_{50}$ for relaxation. (A) and (B) are the same data but expressed in a different manner to illustrate the significant difference in $\mathrm{EC}_{50}$ values between the treatment groups which is clearly seen in (B). The data in (B) is expressed as a percentage of the maximum relaxation response. The urea concentrations shown in the abscissa of the figures refer to the osmolar concentrations of urea added to the IL MKH solution. For urea the osmolarity is the same as the molarity. Saline- and LPS-treated, $n=6$. 


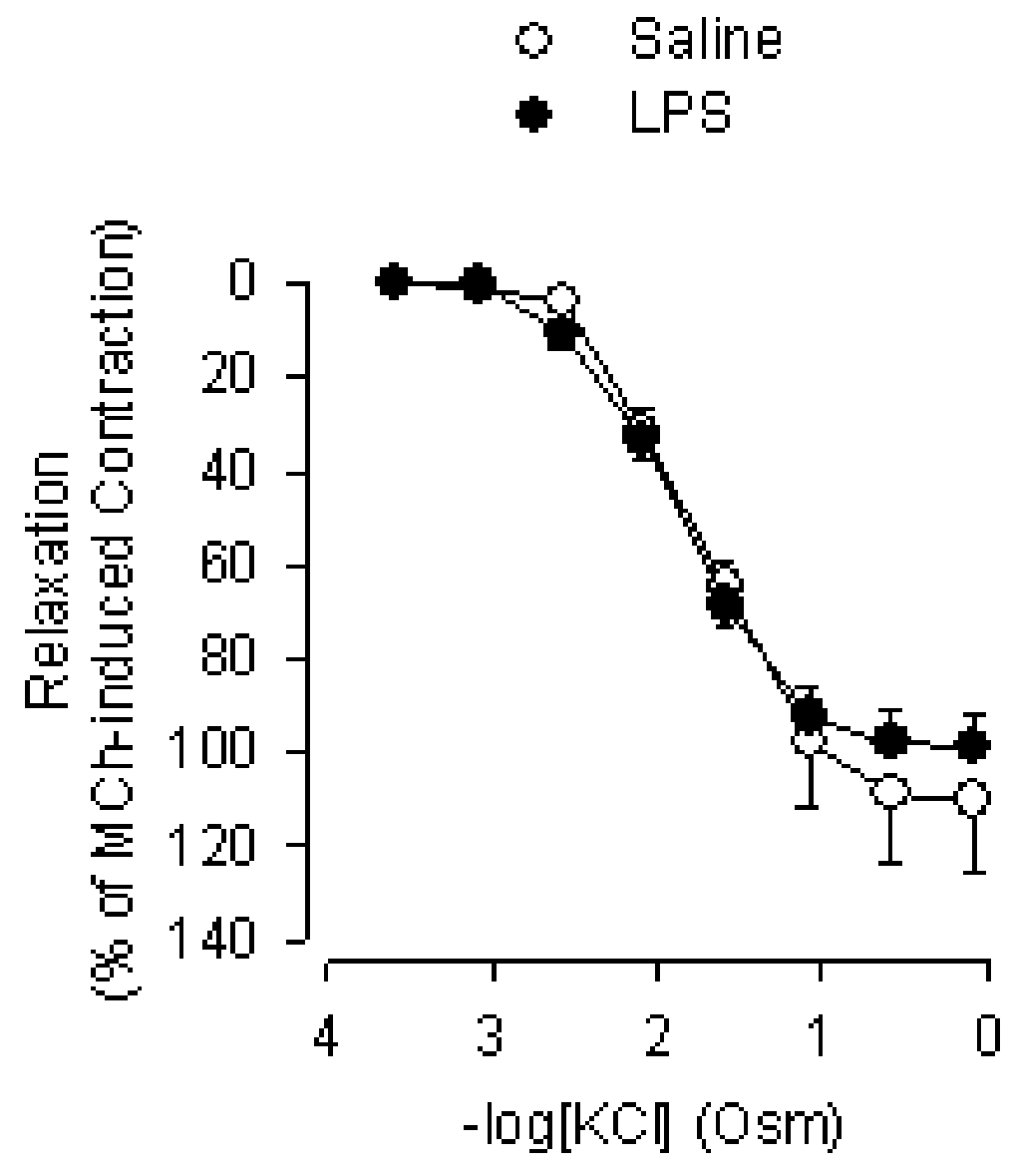

Figure 29. Lack of effect of LPS-treatment on EpDRF-induced smooth muscle relaxation elicited with IL MKH solution made hypertonic with added $\mathrm{KCl}$. The curves were obtained from tracheas removed from saline- and LPS-treated animals $18 \mathrm{hr}$ post-treatment. The preparations were contracted with extraluminally-applied $\mathrm{MCh}\left(3 \times 10^{! 7} \mathrm{M} ; \sim \mathrm{EC}_{50}\right)$ before $\mathrm{KCl}$ was added in stepwise-increasing, cumulative concentrations to the IL bath. Neither the maximum relaxation response nor the $\mathrm{EC}_{50}$ were affected by LPS-treatment when $\mathrm{KCl}$ was used to evoke EpDRF release and subsequent smooth muscle relaxation. The $\mathrm{KCl}$ concentrations shown in the abscissa of the figure refer to the osmolar concentrations of $\mathrm{KCl}$ added to the IL MKH solution. For $\mathrm{KCl}$ the osmolarity is twice the molarity. Saline- and LPS-treated, $n=4$. 
TABLE 1

Effect of LPS-treatment on in vitro airway reactivity to

EpDRF in isolated, perfused trachea

\begin{tabular}{lcc}
\hline Treatment $(n)$ & $\begin{array}{c}\mathrm{EC}_{50}(\mathrm{mOsm})^{a} \\
(95 \% \text { C.I. })\end{array}$ & $\begin{array}{c}\text { Maximum Response } \\
(\% \text { of MCh })^{b}\end{array}$ \\
\hline NaCl & 11.4 & $78.2 \pm 6.2$ \\
Saline (8) & $(8.2-15.8)$ & $113.3 \pm 9.5^{c}$ \\
LPS (8) & 10.4 & \\
D-Mannitol & $(8.4-12.8)$ & $86.1 \pm 4.9$ \\
Saline (6) & 15.2 & $109.7 \pm 8.5^{c}$ \\
LPS (6) & $(8.2-28.2)$ & \\
Urea & 15.9 & $110.3 \pm 7.0$ \\
Saline (6) & $(11.1-22.7)$ & $122.3 \pm 7.5$ \\
LPS (6) & 10.7 & \\
KCl & $(9.0-12.9)$ & $110.2 \pm 15.0$ \\
Saline (4) & $23.3^{c}$ & $99.0 \pm 7.2$ \\
LPS (4) & $(20.2-26.9)$ & \\
\hline
\end{tabular}

The tracheas were removed from the animals $18 \mathrm{hr}$ post-treatment. ${ }^{a}$ Values refer to mOsm of osmolyte added to the intraluminal MKH solution. ${ }^{b}$ Relaxation responses in $\mathrm{cm}_{2} \mathrm{O}$ were normalized as a percentage of the extraluminal $\mathrm{MCh}\left(3 \times 10^{! 7} \mathrm{M} ; \sim \mathrm{EC}_{50}\right)$-induced contraction. ${ }^{c}$ Significantly greater than saline-treated controls. 
Finally, when $\mathrm{KCl}$ was used to evoke EpDRF release and subsequent smooth muscle relaxation, there was no difference in the concentration-response curves among the saline- and LPS-treated groups (Figure 29).

\section{Effect of indomethacin and L-NAME on EpDRF-induced smooth muscle relaxation in isolated, perfused trachea from saline- and LPS-treated guinea pigs}

Because LPS is known to up-regulate both inducible cyclooxygenase and nitric oxide synthase (Okamoto et al., 1998), the effect of indomethacin, a cyclooxygenase inhibitor, and LNAME, a nitric oxide synthase inhibitor, on EpDRF-induced smooth muscle relaxation was tested. This was done to rule out the possible contribution of prostaglandins and NO to the potentiation of EpDRF-induced smooth muscle relaxation following LPS-treatment. Neither indomethacin nor L-NAME affected EpDRF-induced smooth muscle relaxation when $\mathrm{NaCl}$ was used to elevate tonicity in isolated, perfused trachea from saline- and LPS-treated guinea pigs (Figures 30 and 31).

\section{Effect of LPS-treatment on the epithelial bioelectric events associated with EpDRF-induced smooth muscle relaxation}

A previous study has shown that EpDRF-induced smooth muscle relaxation, elicited by hypertonicity, was associated with epithelial bioelectric events, namely a depolarization of the $\mathrm{V}_{\mathrm{ms}}$ (Dortch-Carnes et al., 1999). The current study has shown that LPS-treatment potentiated EpDRF-induced smooth muscle relaxation when $\mathrm{NaCl}$ and D-mannitol were used to elicit EpDRF release; therefore, according to the hypothesis, the epithelial bioelectric events associated with increased EpDRF-induced smooth muscle relaxation should also be potentiated following LPS-treatment. 


\section{- Indomethacin \\ - Hndomethacin}
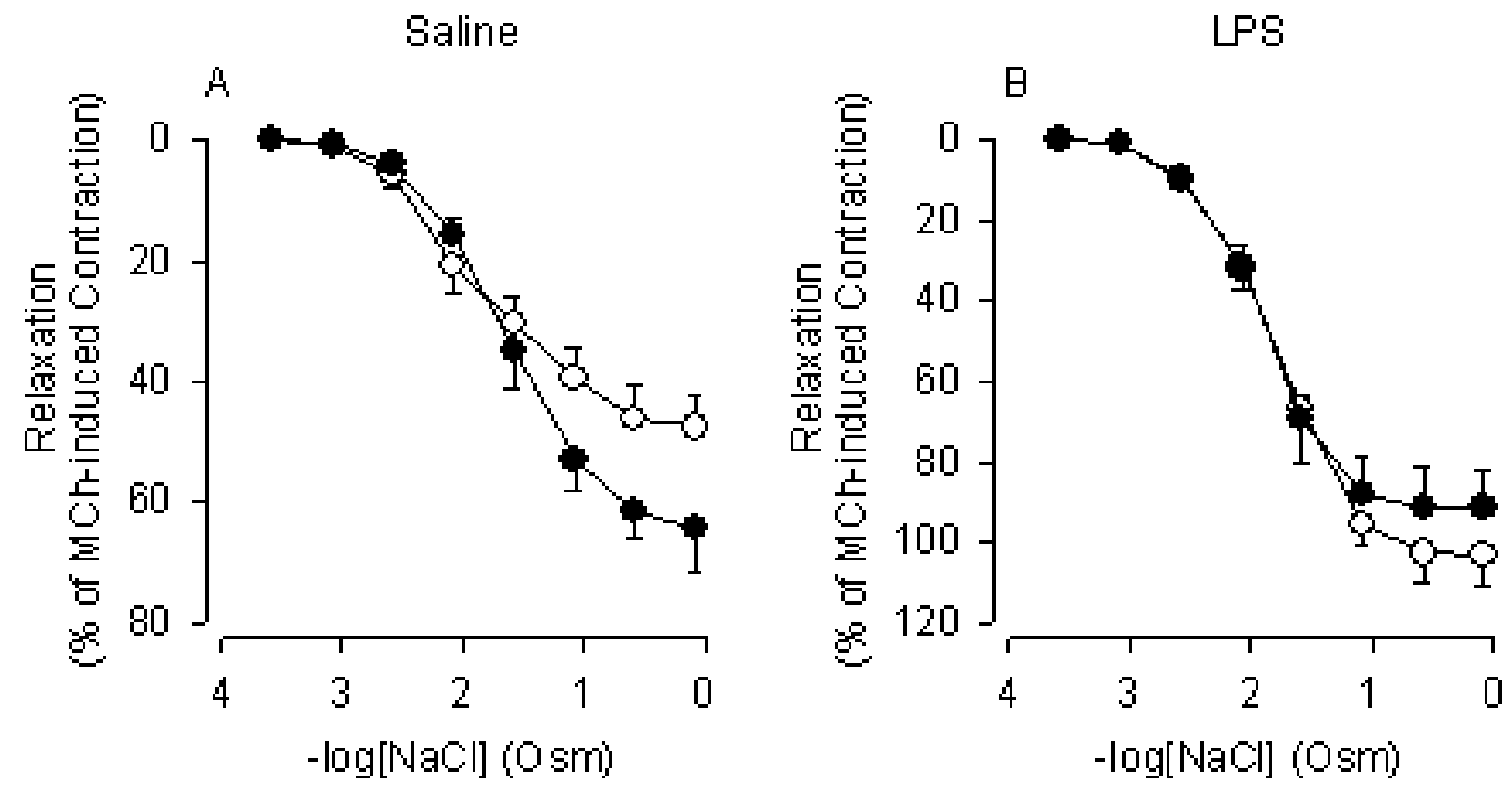

Figure 30. Lack of effect of extraluminally- and intraluminally-applied indomethacin $\left(3 \times 10^{!} 6\right.$ M) on EpDRF-mediated relaxation responses elicited with IL MKH solution made hypertonic with added $\mathrm{NaCl}$. The curves were obtained from tracheas removed from (A) saline- and (B) LPS-treated animals $18 \mathrm{hr}$ post-treatment. Indomethacin was present for $30 \mathrm{~min}$ prior to contracting the tracheal smooth muscle with extraluminally-applied $\mathrm{MCh}\left(3 \times 10^{! 7} \mathrm{M} ; \sim \mathrm{EC}_{50}\right)$ and initiating EpDRF-induced smooth muscle relaxation by adding $\mathrm{NaCl}$ in stepwise-increasing, cumulative concentrations to the IL bath. Saline- and LPS-treated, $n=4$. 


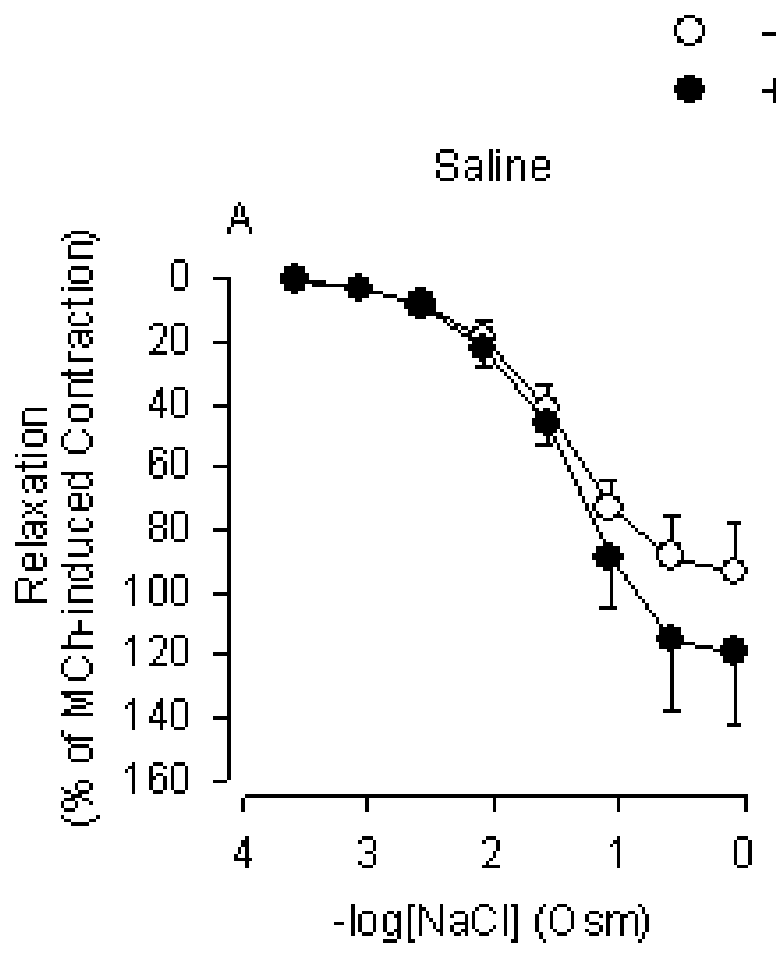

-L-NAME

HL-NAME

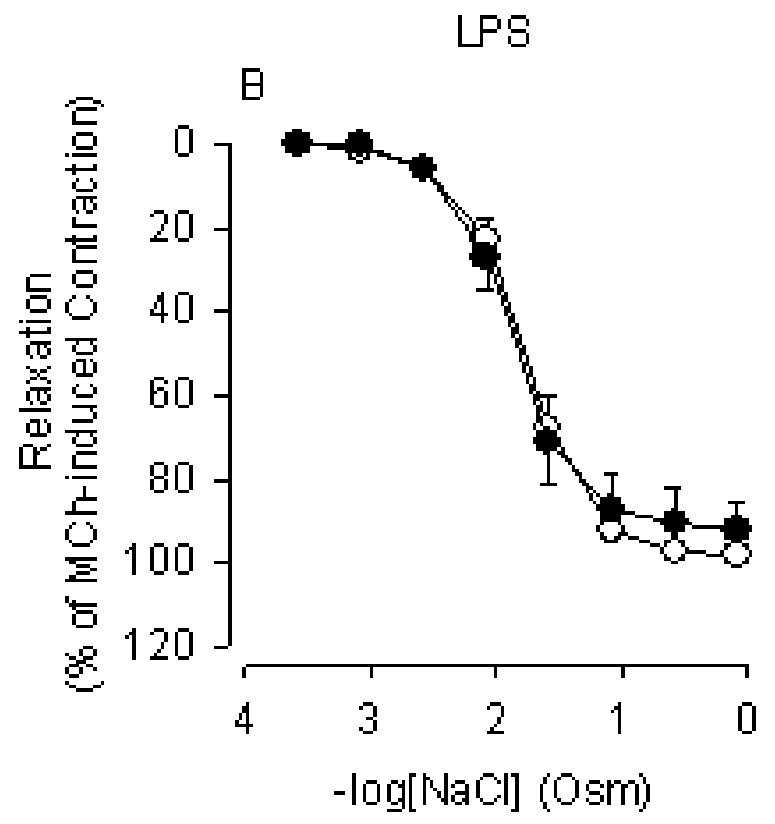

Figure 31. Lack of effect of extraluminally- and intraluminally-applied L-NAME $\left(10^{! 4} \mathrm{M}\right)$ on EpDRF-mediated relaxation responses elicited with IL MKH solution made hypertonic with added $\mathrm{NaCl}$. The curves were obtained from tracheas removed from (A) saline- and (B) LPStreated animals $18 \mathrm{hr}$ post-treatment. L-NAME was present for $30 \mathrm{~min}$ prior to contracting the tracheal smooth muscle with extraluminally-applied $\mathrm{MCh}\left(3 \times 10^{! 7} \mathrm{M} ; \sim \mathrm{EC}_{50}\right)$ and initiating EpDRF-induced smooth muscle relaxation by adding $\mathrm{NaCl}$ in stepwise-increasing, cumulative concentrations to the IL bath. Saline- and LPS-treated, $n=4$. 
$\mathrm{NaCl}$ concentration-response curves with respect to $\triangle \mathrm{V}_{\mathrm{ms}}$ were generated from isolated, perfused tracheas from saline- and LPS-treated animals in the presence of extraluminally-applied $3 \times 10^{! 7} \mathrm{M} \mathrm{MCh}$ (Figure 32 ). For an easy comparison of the relationship between hypertonicityinduced epithelial depolarization and smooth muscle relaxation, depolarization and relaxation concentration-response curves were plotted alongside each other as a percentage of the maximum depolarization or relaxation, respectively (Figure 33). In tracheas from LPS-treated animals, the IL addition of $\mathrm{NaCl}$ significantly increased epithelial depolarization and EpDRFinduced smooth muscle relaxation compared to the saline-treated controls; however, the $\mathrm{V}_{\mathrm{ms}}$ of each treatment group eventually depolarized to the same level (Figure 32 and Table 2). In both treatment groups, the $\mathrm{EC}_{50}$ for depolarization was significantly greater than the $\mathrm{EC}_{50}$ for $\mathrm{EpDRF}$ induced smooth muscle relaxation (Figure 33 and Table 2).

Besides examining epithelial bioelectric reactivity to $\mathrm{NaCl}$ in the presence of extraluminally-applied MCh $\left(3 \times 10^{! 7} \mathrm{M}\right)$, this study also examined epithelial bioelectric reactivity to $\mathrm{NaCl}$ in the absence of $\mathrm{MCh}$ (Figures 34 and 35 and Table 3). The addition of hypertonic $\mathrm{NaCl}$ to the $\mathrm{EL}$ and $\mathrm{IL}$ baths depolarized the $\mathrm{V}_{\mathrm{ms}}$ in a concentration-dependent manner. In both treatment groups, extraluminally-applied $\mathrm{NaCl}$ was more potent than intraluminally-applied $\mathrm{NaCl}$. When comparing responses between the two treatment groups, the $\mathrm{IL} \mathrm{EC}_{50}$ for depolar-ization was significantly greater in the LPS-treated group compared to the saline-treated group. Finally, extraluminally- and intraluminally-applied $\mathrm{NaCl}$ depolarized the $\mathrm{V}_{\mathrm{ms}}$ to a greater extent in the LPS-treated group; however, it did not reach statistical significance, except for one concentration added intraluminally (Figure 35). 


\section{o Saline \\ - LPS}
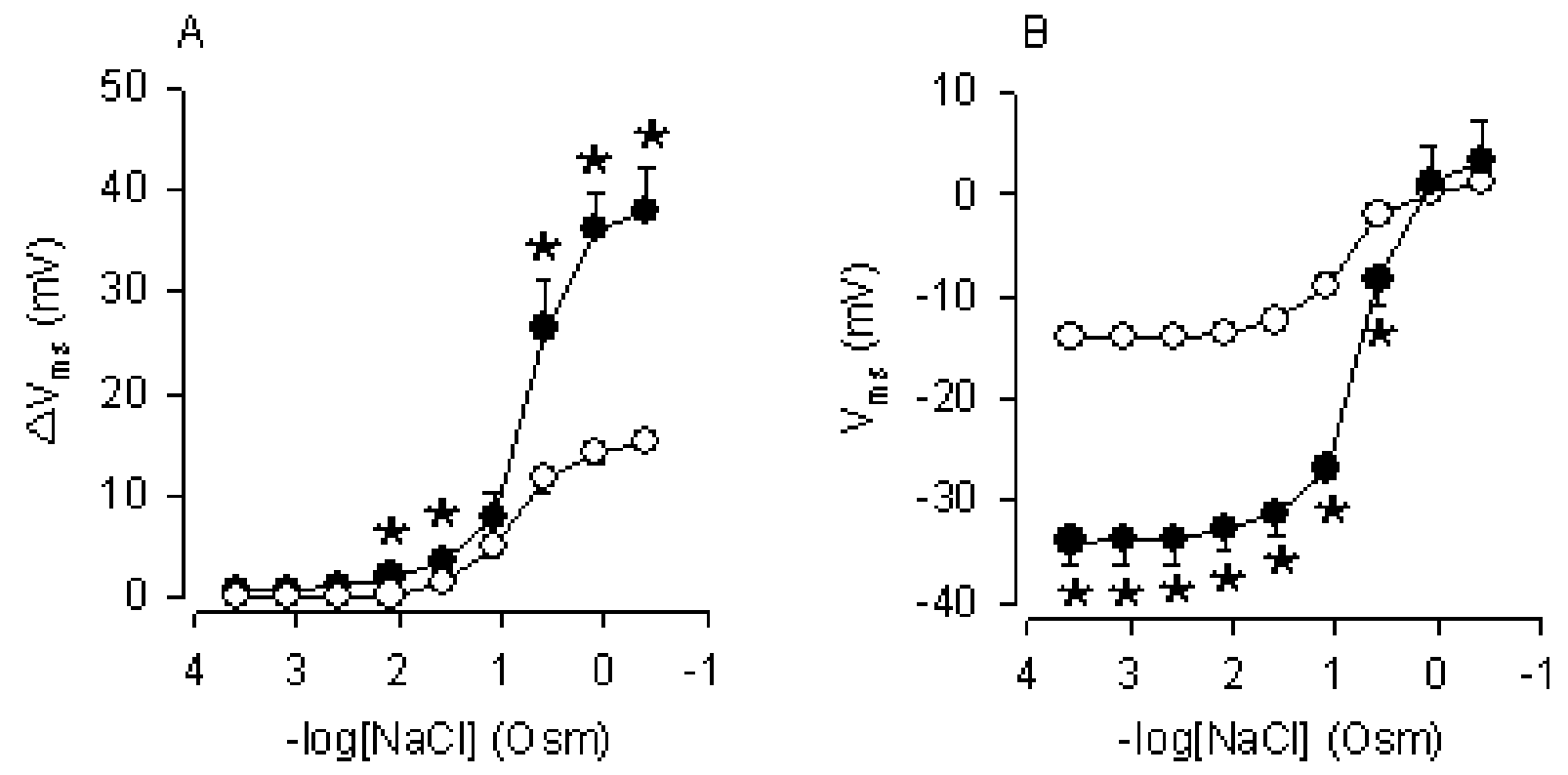

Figure 32. Effect of LPS-treatment on epithelial bioelectric reactivity to hypertonic $\mathrm{NaCl}$ in the presence of extraluminally-added $\mathrm{MCh}\left(3 \times 10^{! 7} \mathrm{M}\right)$. The curves were obtained from tracheas removed from saline- and LPS-treated animals $18 \mathrm{hr}$ post-treatment. $\mathrm{MCh}\left(3 \times 10^{!} \mathrm{M}\right)$ was added to the EL bath before $\mathrm{NaCl}$ was added in stepwise-increasing, cumulative concentrations to the IL bath. (A) The $\mathrm{mV}$ change in the $\mathrm{V}_{\mathrm{ms}}$ evoked by the addition of IL NaCl. LPS-treatment significantly increased the degree of depolarization while the $\mathrm{EC}_{50}$ 's were unaffected. (B) These are data from the same experiments in (A); however, they are expressed in a different manner. These plots show the actual $\mathrm{V}_{\mathrm{ms}}$ following the addition of IL $\mathrm{NaCl}$. In both treatment groups, intraluminally-applied $\mathrm{NaCl}$ eventually depolarized the $\mathrm{V}_{\mathrm{ms}}$ to the same level. Saline- and LPStreated, $n=4$. ${ }^{*} p<0.05$ compared to saline-treated as determined by a nonpaired Student's $t$ test. 


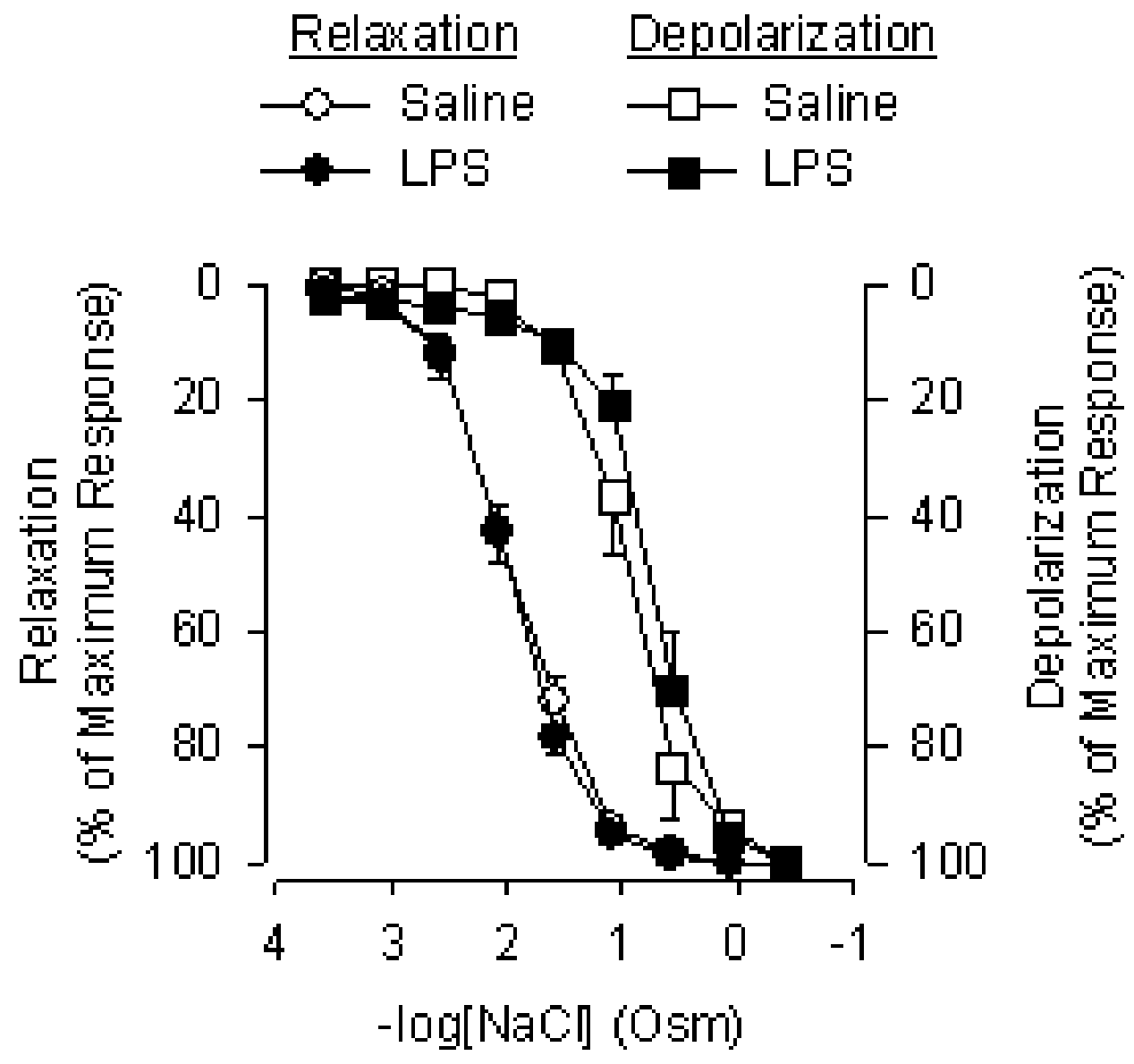

Figure 33. Effect of LPS-treatment on the relationship between EpDRF-induced smooth muscle relaxation and epithelial depolarization elicited by hypertonic $\mathrm{NaCl}$. The curves were obtained from tracheas removed from saline- and LPS-treated animals $18 \mathrm{hr}$ post-treatment. The preparations were contracted with extraluminally-applied $\mathrm{MCh}\left(3 \times 10^{! 7} \mathrm{M} ; \sim \mathrm{EC}_{50}\right)$ before $\mathrm{NaCl}$ was added in stepwise-increasing, cumulative concentrations to the IL bath. This figure illustrates that the $\mathrm{EC}_{50}$ 's for depolarization are significantly greater than the $\mathrm{EC}_{50}$ 's for relaxation in both treatment groups. Saline- and LPS-treated for relaxation, $n=8$ and saline- and LPStreated for depolarization, $n=4$. 
TABLE 2

Relationship between in vitro smooth muscle relaxation and epithelial depolarization in isolated, perfused trachea from saline- and LPS-treated guinea pigs

\begin{tabular}{lcccc}
\hline & \multicolumn{2}{c}{$\mathrm{EC}_{50}(\mathrm{mOsm})^{a}$} & \multicolumn{2}{c}{ Maximum Response } \\
\cline { 2 - 5 } Treatment & $\begin{array}{c}\text { Relaxation } \\
(\% \text { of } \mathrm{MCh})^{b}\end{array}$ & $\begin{array}{c}\text { Depolarization } \\
\triangle \mathrm{V}_{\mathrm{ms}}(\mathrm{mV})^{c}\end{array}$ & $\begin{array}{c}\text { Relaxation } \\
(\% \text { of } \mathrm{MCh})^{b}\end{array}$ & $\begin{array}{c}\text { Depolarization } \\
\mathrm{V}_{\mathrm{ms}}(\mathrm{mV})^{c}\end{array}$ \\
\hline Saline & 11.4 & $121.6^{d}$ & $78.2 \pm 6.2$ & $15.3 \pm 0.5$ \\
LPS & $(8.2-15.8)$ & $(75.0-197.2)$ & & \\
& 10.4 & $183.6^{e}$ & $113.3 \pm 9.5^{f}$ & $38.0 \pm 4.1^{f}$ \\
& $(8.4-12.8)$ & $(120.2-280.4)$ & & \\
\hline
\end{tabular}

The tracheas were removed from the animals $18 \mathrm{hr}$ post-treatment. ${ }^{a}$ Values refer to mOsm of $\mathrm{NaCl}$ added to the intraluminal $\mathrm{MKH}$ solution. ${ }^{b}$ Relaxation responses in $\mathrm{cm}_{2} \mathrm{O}$ were normalized as a percentage of the extraluminal $\mathrm{MCh}\left(3 \times 10^{! 7} \mathrm{M} ; \sim \mathrm{EC}_{50}\right)$-induced contraction; saline- and LPS- treated, $n=8$. ${ }^{c}$ Values refer to the change of the $\mathrm{V}_{\mathrm{ms}}$ after the administration of extraluminal MCh $\left(3 \times 10^{! 7} \mathrm{M}\right)$; saline- and LPS-treated, $n=4$. ${ }^{d}$ Significantly greater than saline (relaxation). ${ }^{e}$ Significantly greater than LPS (relaxation). ${ }^{f}$ Significantly greater than salinetreated controls. 


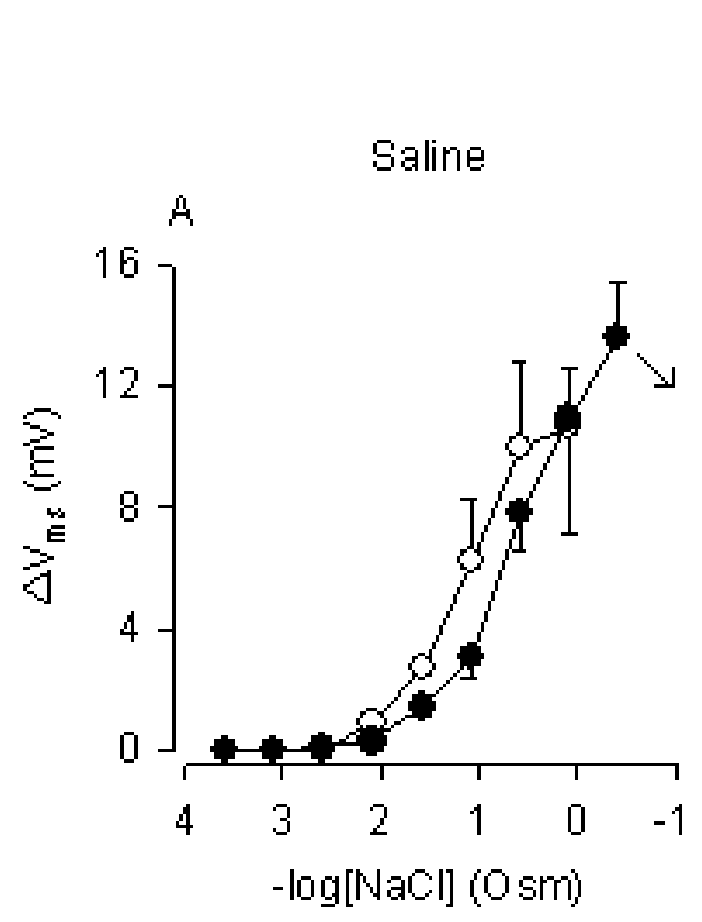

\section{Extraluminal \\ * Intraluminal}

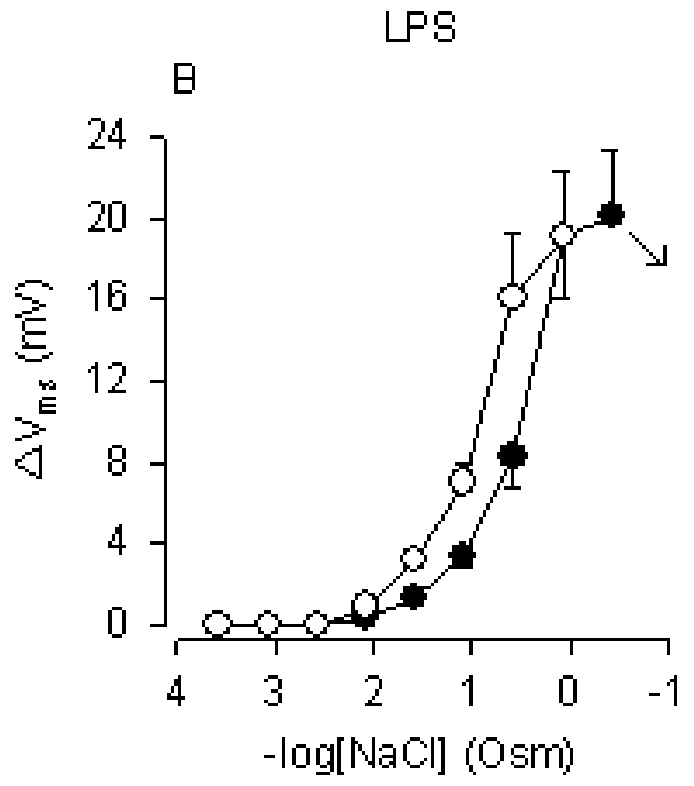

Figure 34. Effect of LPS-treatment on epithelial bioelectric reactivity to extraluminally- and intraluminally-applied $\mathrm{NaCl}$ in the absence of $\mathrm{MCh}$ (a within-treatment group comparison). EL and IL $\mathrm{NaCl}$ concentration-response curves were obtained from tracheas removed from (A) saline- and (B) LPS-treated animals $18 \mathrm{hr}$ post-treatment. $\mathrm{NaCl}$ was added in stepwiseincreasing, cumulative concentrations to the EL and IL baths. In both treatment groups, extraluminally-applied $\mathrm{NaCl}$ was more potent. The arrows refer to the fact that the next highest concentration of intraluminally-applied $\mathrm{NaCl}$ reversed the depolarization, or in other words, hyperpolarized the $\mathrm{V}_{\mathrm{ms}}$. In addition, because of the size of the EL bath and the limits of solubility of $\mathrm{NaCl}$, only $843.4 \mathrm{mOsm}$ of $\mathrm{NaCl}$ could be added. Saline-treated, $n=7$ and LPStreated, $n=6$. 


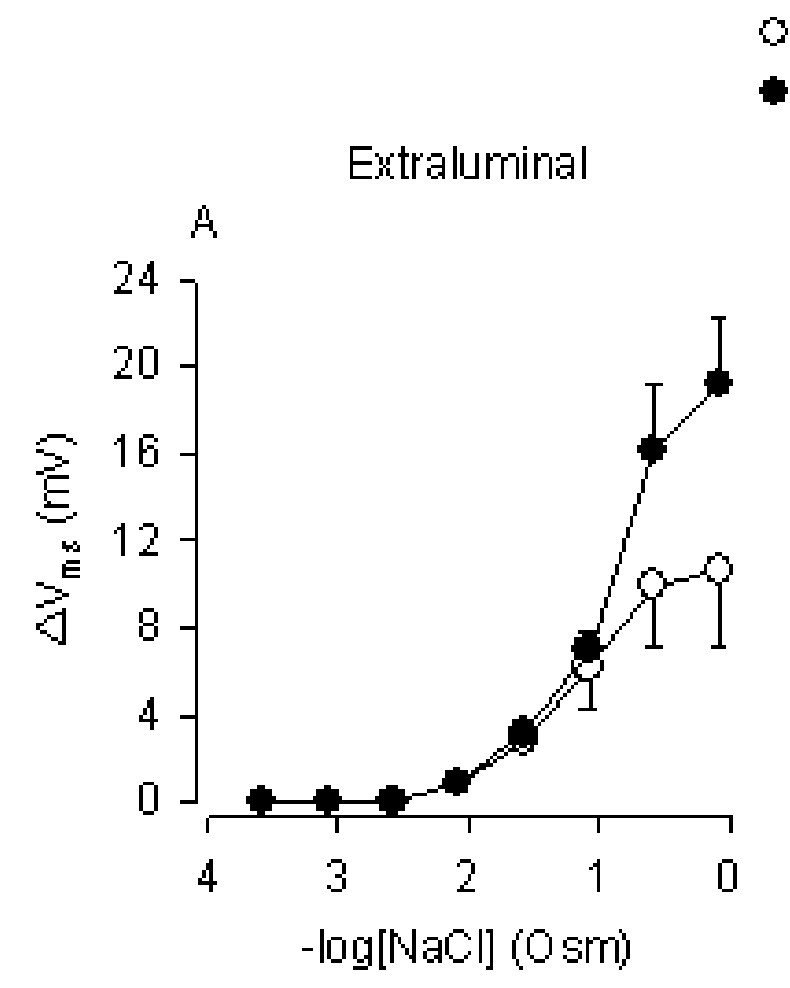

\section{o Saline \\ - LPS}

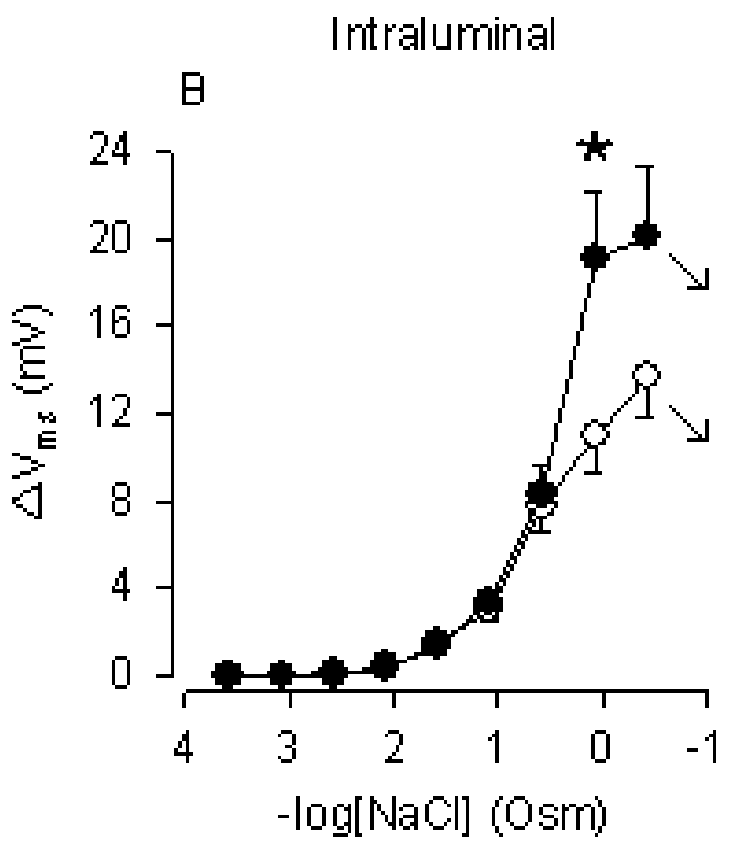

Figure 35. Effect of LPS-treatment on epithelial bioelectric reactivity to (A) extraluminally- and (B) intraluminally-applied $\mathrm{NaCl}$ in the absence of $\mathrm{MCh}$ (a between-treatment group comparison). This is the same data shown in Figure 34 but in a different manner. EL and IL NaCl concentration-response curves were obtained from tracheas removed from saline- and LPStreated animals $18 \mathrm{hr}$ post-treatment. $\mathrm{NaCl}$ was added in stepwise-increasing, cumulative concentrations to the EL and IL baths. $\mathrm{NaCl}$ depolarized the $\mathrm{V}_{\mathrm{ms}}$ to a greater extent in the LPStreated group; however, it did not reach statistical significance, except for one concentration added intraluminally. The arrows refer to the fact that the next highest concentration of intraluminally-applied $\mathrm{NaCl}$ reversed the depolarization, or in other words, hyperpolarized the $\mathrm{V}_{\mathrm{ms}}$. In addition, because of the size of the EL bath and the limits of solubility of $\mathrm{NaCl}$, only $843.4 \mathrm{mOsm}$ of $\mathrm{NaCl}$ could be added. Saline-treated, $n=7$ and LPS-treated, $n=6 .{ }^{*} p<0.05$ compared to saline-treated as determined by a nonpaired Student's $t$ test. 
TABLE 3

Effect of LPS-treatment on in vitro epithelial bioelectric reactivity to hypertonic $\mathrm{NaCl}$ in isolated, perfused trachea

\begin{tabular}{lcc}
\hline & \multicolumn{2}{c}{$\mathrm{EC}_{50}(\mathrm{mOsm})^{a}$} \\
& & \multicolumn{2}{c}{$(95 \%$ C.I. $)$} \\
\cline { 2 - 3 } Treatment $(n)$ & EL & IL \\
\hline Saline (7) & 68.2 & $157.4^{b}$ \\
LPS (6) & $(57.6-81.0)$ & $(95.0-261.0)$ \\
& 88.6 & $313.4^{c, d}$ \\
& $(73.0-107.2)$ & $(283.0-347.0)$ \\
\hline
\end{tabular}

EL, extraluminal; IL, intraluminal. The tracheas were removed from the animals $18 \mathrm{hr}$ posttreatment. ${ }^{a}$ Values refer to $\mathrm{mOsm}$ of $\mathrm{NaCl}$ added to the extraluminal or intraluminal $\mathrm{MKH}$ solution. ${ }^{b}$ Significantly greater than saline (EL). ${ }^{c}$ Significantly greater than LPS (EL). ${ }^{d}$ Significantly greater than saline (IL). 


\section{Effects of indomethacin and L-NAME on LPS-induced alterations of the basal $V_{m s}$ in isolated, perfused trachea}

In order to elucidate the mechanism underlying the increase in the basal $\mathrm{V}_{\mathrm{ms}}$ following LPS-treatment, the effect of indomethacin $\left(3 \times 10^{! 6} \mathrm{M}\right)$ and L-NAME $\left(10^{! 4} \mathrm{M}\right)$ on the basal $\mathrm{V}_{\mathrm{ms}}$ was examined. Both indomethacin and L-NAME were chosen because LPS up-regulates their targets, cyclooxygenase and nitric oxide synthase (Okamoto et al., 1998) whose products, prostaglandins and NO, respectively, have been shown to stimulate $\mathrm{Cl}^{\text {! }}$ secretion (Tamaoki et al., 1992; Tamai and Gaginella, 1993).

Indomethacin depolarized the $\mathrm{V}_{\mathrm{ms}}$ in both treatment groups (Figure 36), but significantly more so in the LPS-treated group: $+3.1 \pm 0.5 \mathrm{mV}$ and $+5.5 \pm 0.7 \mathrm{mV}$ for the saline- and LPStreated groups, respectively $(p<0.05)$.

L-NAME slightly hyperpolarized the $\mathrm{V}_{\mathrm{ms}}$ in both treatment groups (Figure 37); however, there was no significant difference between the magnitude of the hyperpolarization among the saline- and LPS-treated groups.

Because L-NAME has been shown to possess muscarinic receptor antagonist properties (Buxton et al., 1993), the effect of atropine $\left(10^{! 6} \mathrm{M}\right)$, a muscarinic receptor antagonist, on the basal $\mathrm{V}_{\mathrm{ms}}$ was characterized to determine if the small effect of L-NAME was due to the inhibition of nitric oxide synthesis or muscarinic receptor blockade. 


\section{Indomethacin}
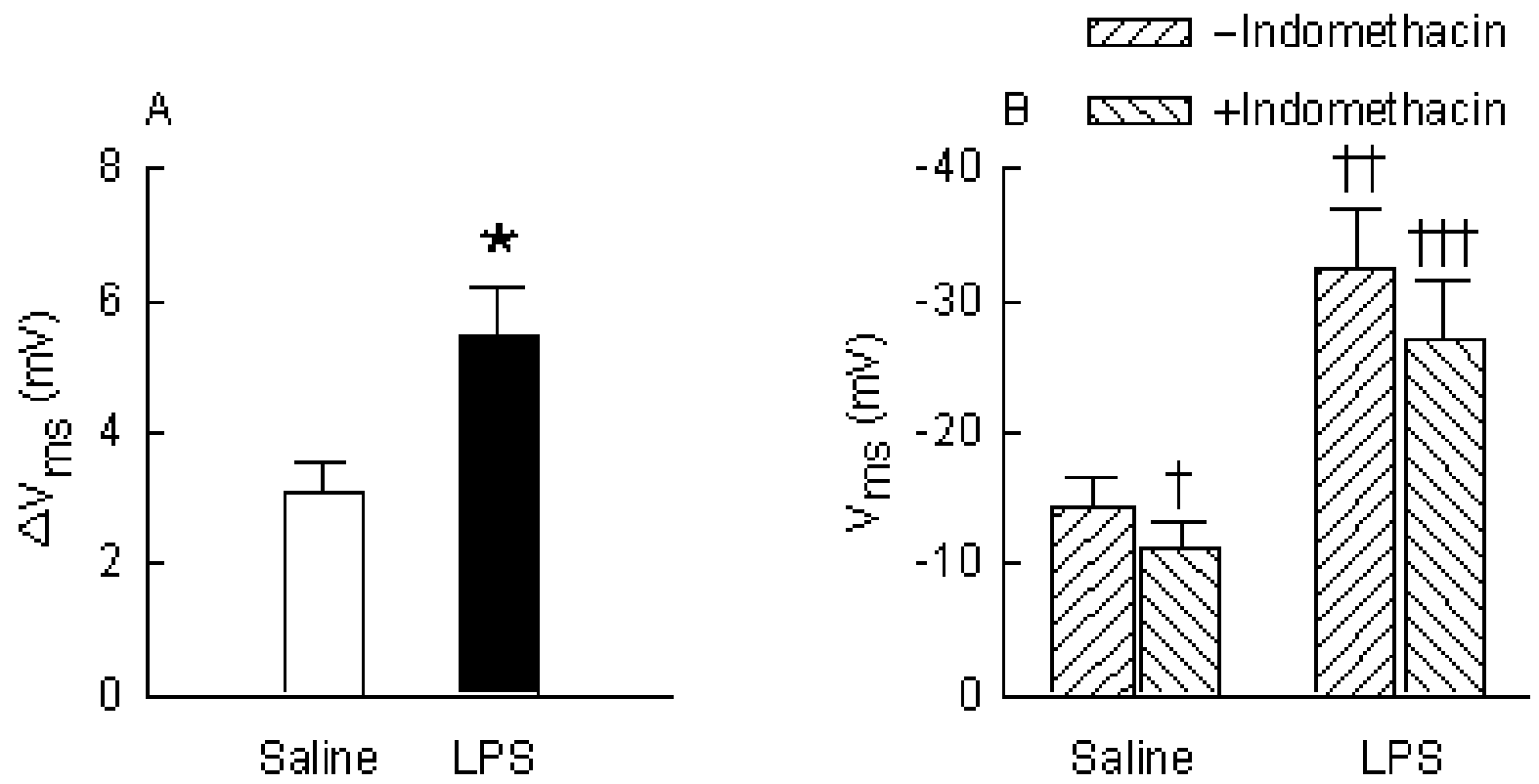

Figure 36. Effect of indomethacin on the basal $\mathrm{V}_{\mathrm{ms}}$ of isolated, perfused trachea from salineand LPS-treated animals. The tracheas were removed from saline- and LPS-treated animals 18 hr post-treatment. Following a $3 \mathrm{hr}$ equilibration period, indomethacin $\left(3 \times 10^{! 6} \mathrm{M}\right)$ was simultaneously added to the EL and IL baths. (A) The actual $\mathrm{mV}$ change in the basal $\mathrm{V}_{\mathrm{ms}}$ evoked by the addition of indomethacin. The depolarizing effect of indomethacin was significantly greater in the LPS-treated group. (B) In the presence of indomethacin, the $\mathrm{V}_{\mathrm{ms}}$ was significantly decreased in both treatment groups. Saline- and LPS-treated, $n=5$. ${ }^{*} p<0.05$ compared to saline-treated as determined by a nonpaired Student's $t$ test. $\dagger p<0.05$ compared to saline-treated (! Indomethacin) as determined by a paired Student's $t$ test. $\dagger \dagger p<0.05$ compared to saline-treated (! Indomethacin) as determined by a nonpaired Student's $t$ test. $\dagger \dagger \uparrow p<0.05$ compared to LPS-treated (! Indomethacin) as determined by a paired Student's $t$ test. 

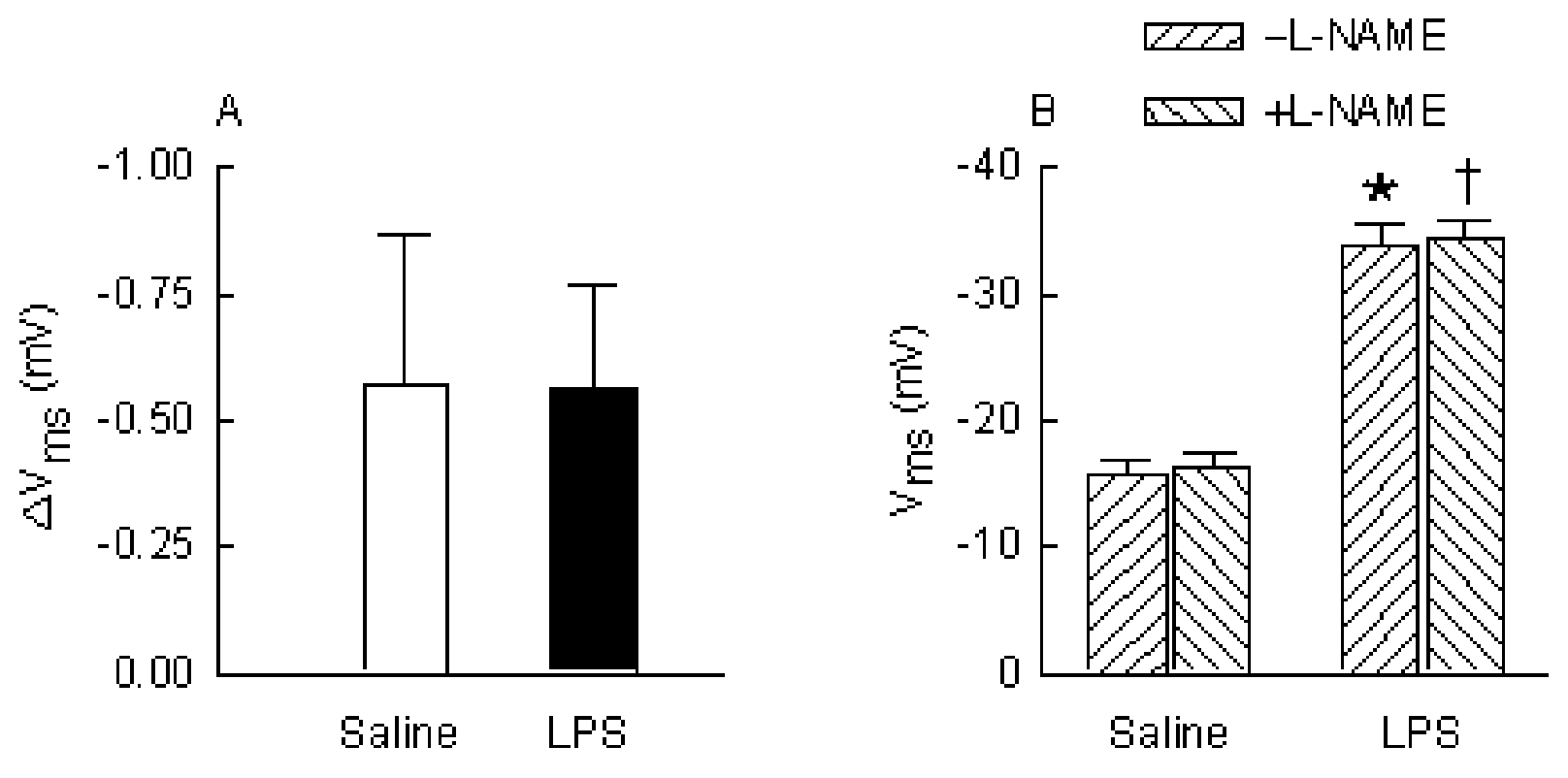

Figure 37. Effect of L-NAME on the basal $\mathrm{V}_{\mathrm{ms}}$ of isolated, perfused trachea from saline- and LPS-treated animals. The tracheas were removed from saline- and LPS-treated animals $18 \mathrm{hr}$ post-treatment. Following a $3 \mathrm{hr}$ equilibration period, L-NAME $\left(10^{! 4} \mathrm{M}\right)$ was added simultaneously to the EL and IL baths. (A) The actual $\mathrm{mV}$ change in the $\mathrm{V}_{\mathrm{ms}}$ evoked by the addition of L-NAME. There was no difference in the hyperpolarizing effect of L-NAME between the treatment groups. (B) There was no difference in the $\mathrm{V}_{\mathrm{ms}}$ before and after the addition of L-NAME within each treatment group. Saline- and LPS-treated, $n=4$. $* p<0.05$ compared to saline-treated (! L-NAME) as determined by a nonpaired Student's $t$ test. $\dagger p<0.05$ compared to saline-treated (+L-NAME) as determined by a nonpaired Student's $t$ test. 
The effect of atropine on the basal $\mathrm{V}_{\mathrm{ms}}$ in isolated, perfused trachea from saline- and LPStreated animals is shown in Figure 38. Atropine's effect was variable in both treatment groups. Atropine initiated either a depolarization or a hyperpolarization of the $\mathrm{V}_{\mathrm{ms}}$ which did not exceed $\pm 2 \mathrm{mV}$.

Since atropine did not clarify the effects of nitric oxide and its possible contribution to the basal $\mathrm{V}_{\mathrm{ms}}$, the effect of sodium nitroprusside (SNP), a nitric oxide donor, on the basal $\mathrm{V}_{\mathrm{ms}}$ of isolated, perfused trachea from saline- and LPS-treated animals was determined (Figure 39). SNP $\left(10^{! 6} \mathrm{M}\right)$ caused a very small depolarization of the basal $\mathrm{V}_{\mathrm{ms}}$; however, there was no difference in the magnitude of the depolarization among the treatment groups.

\section{Effect of amiloride on LPS-induced alterations of the basal $V_{m s}$ in isolated, perfused trachea}

Since the effects of indomethacin and L-NAME gave little insight into the mechanism responsible for the increased basal $\mathrm{V}_{\mathrm{ms}}$ in tracheas from LPS-treated animals, the $\mathrm{Na}^{+}$channel blocker, amiloride, was used (Figure 40). Amiloride was chosen because its target plays an

important role in epithelial ion transport (Figure 6). The application of amiloride $\left(3 \times 10^{!} 5 \mathrm{M}\right)$ to the IL surface of isolated, perfused tracheas from saline- and LPS-treated animals depolarized the $\mathrm{V}_{\mathrm{ms}}$, but significantly more so in the LPS-treated group. After the addition of amiloride, there was no longer any difference between the $\mathrm{V}_{\mathrm{ms}}$ 's among the saline- and LPS-treated groups. 


\section{Atropine}

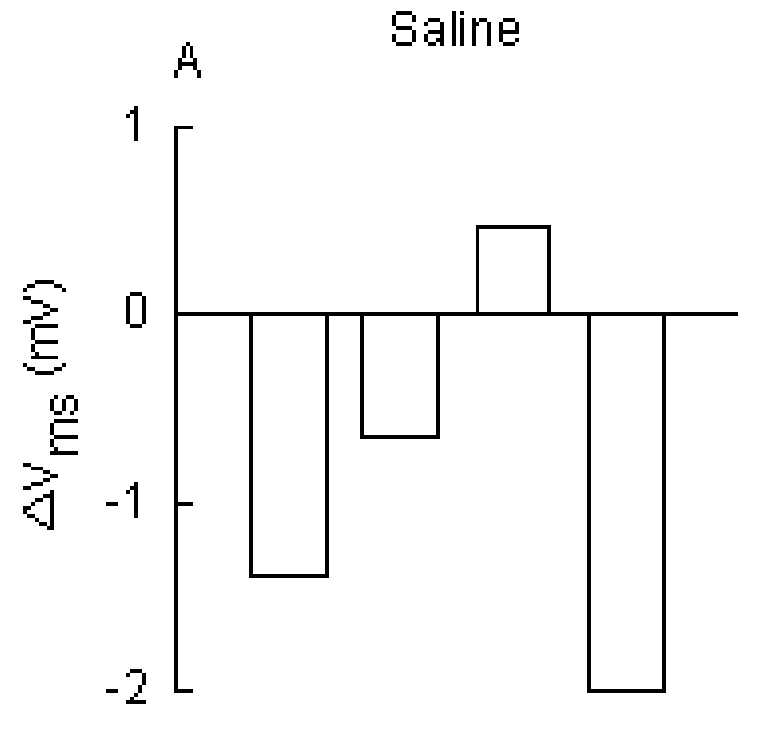

$\bar{x}=-0.89 \pm 0.53$

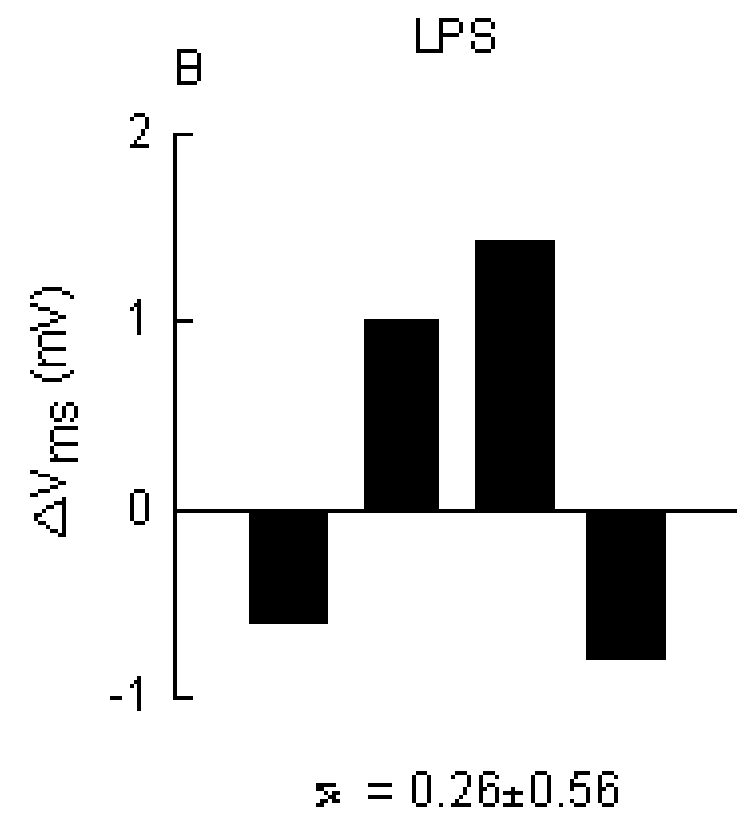

Figure 38. Effect of atropine on the basal $\mathrm{V}_{\mathrm{ms}}$ of isolated, perfused trachea from (A) saline- and (B) LPS-treated animals. The tracheas were removed from saline- and LPS-treated animals 18 hr post-treatment. Following a $3 \mathrm{hr}$ equilibration period, atropine $\left(10^{! 6} \mathrm{M}\right)$ was added simultaneously to the EL and IL baths. In both groups, atropine had variable effects, sometimes depolarizing and other times hyperpolarizing. Since there was no consistent effect, the raw data from four individual experiments was plotted and an average value shown underneath each graph. Saline- and LPS-treated, $n=4$. 


\section{Sodium Nitroprusside (SNP)}
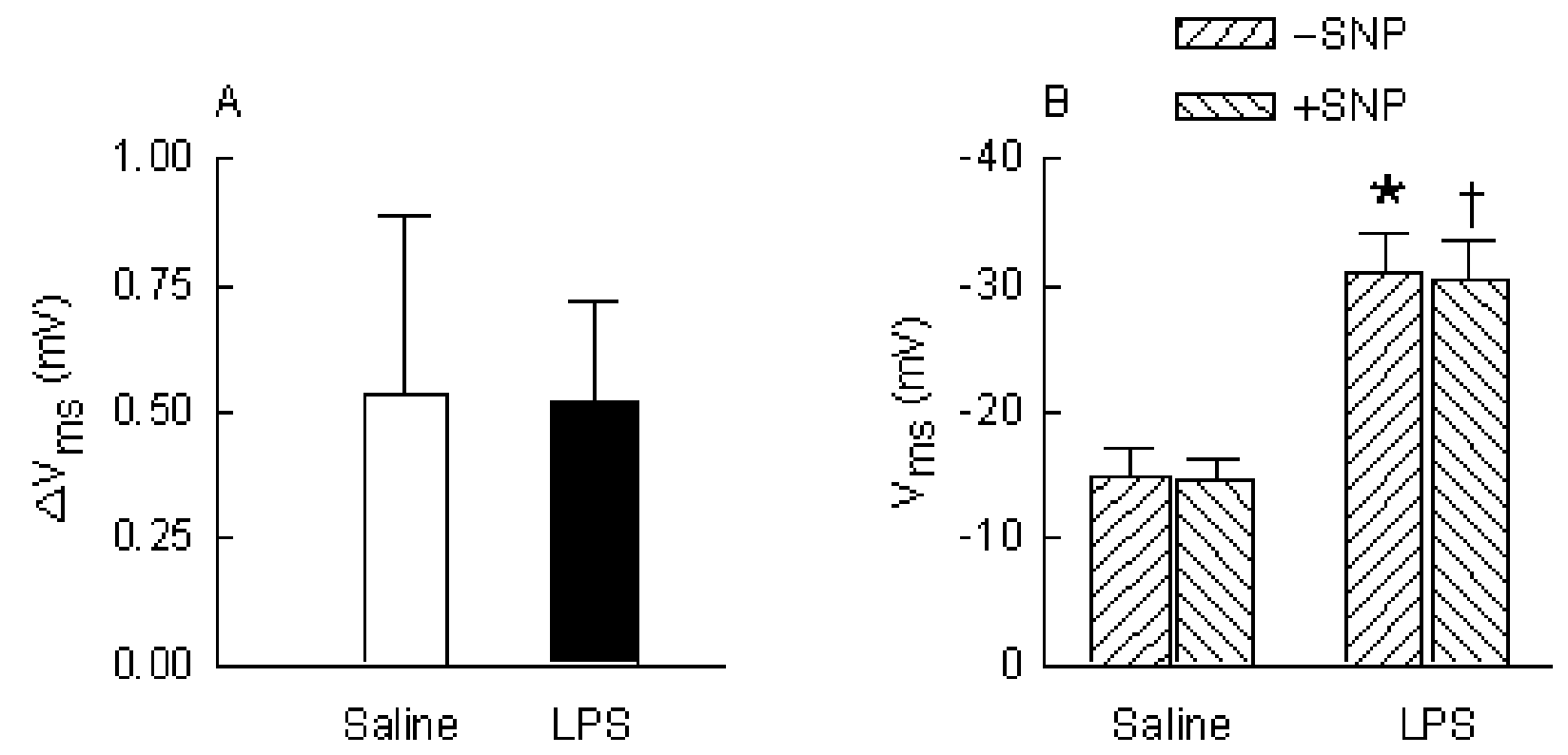

Figure 39. Effect of SNP on the basal $\mathrm{V}_{\mathrm{ms}}$ of isolated, perfused trachea from saline- and LPStreated animals. The tracheas were removed from saline- and LPS-treated animals $18 \mathrm{hr}$ posttreatment. Following a $3 \mathrm{hr}$ equilibration period, SNP $\left(10^{! 6} \mathrm{M}\right)$ was added to the EL and IL baths. (A) The actual $\mathrm{mV}$ change in the basal $\mathrm{V}_{\mathrm{ms}}$ evoked by the addition of SNP. There was no significant difference between the degree to which SNP depolarized the $V_{m s}$ among the treatment groups. (B) There was no difference in the $\mathrm{V}_{\mathrm{ms}}$ before and after the addition of SNP within each treatment group. Saline- and LPS-treated, $n=3 .{ }^{*} p<0.05$ compared to saline-treated (! SNP) as determined by a nonpaired Student's $t$ test. $\dagger p<0.05$ compared to saline-treated (+SNP) as determined by a nonpaired Student's $t$ test. 


\section{Amiloride}
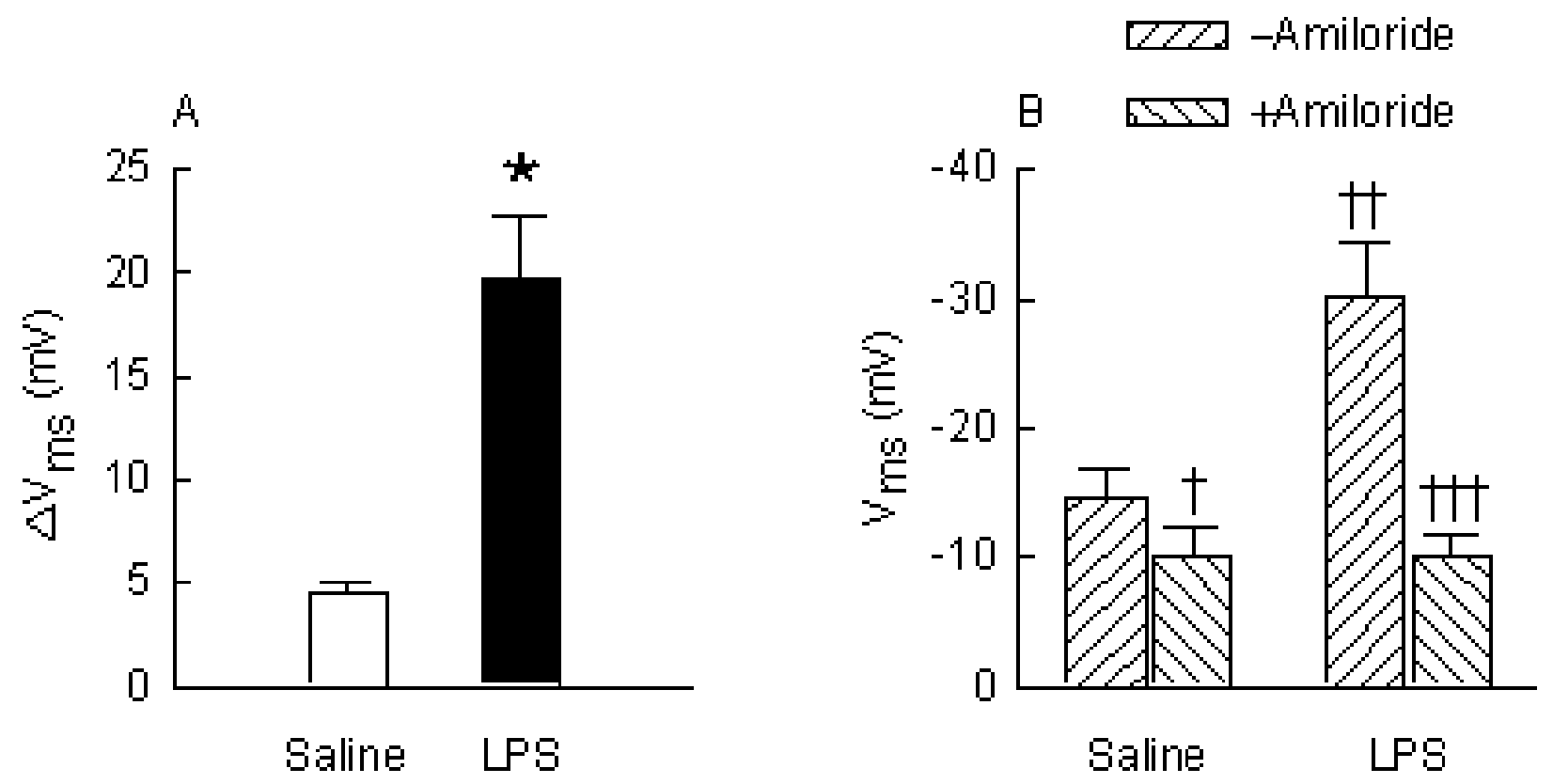

Figure 40. Effect of amiloride on the basal $\mathrm{V}_{\mathrm{ms}}$ of isolated, perfused trachea from saline- and LPS-treated animals. The tracheas were removed from saline- and LPS-treated animals $18 \mathrm{hr}$ post-treatment. Following a $3 \mathrm{hr}$ equilibration period, amiloride $\left(3 \times 10^{! 5} \mathrm{M}\right)$ was added to the IL bath. (A) The actual $\mathrm{mV}$ change in the basal $\mathrm{V}_{\mathrm{ms}}$ evoked by the addition of amiloride. The depolarizing effect of amiloride was significantly greater in the LPS-treated group. (B) Amiloride significantly altered the $\mathrm{V}_{\mathrm{ms}}$ in both treatment groups. After the IL application of amiloride, there was no longer a difference between the $\mathrm{V}_{\mathrm{ms}}$ 's among the two groups. Salineand LPS-treated, $n=5$. ${ }^{*} p<0.05$ compared to saline-treated as determined by a nonpaired Student's $t$ test. $\dagger p<0.05$ compared to saline-treated (! Amiloride) as determined by a paired Student's $t$ test. $\dagger \dagger p<0.05$ compared to saline-treated (! Amiloride) as determined by a nonpaired Student's $t$ test. $\dagger \dagger \uparrow p<0.05$ compared to LPS-treated (! Amiloride) as determined by a paired Student's $t$ test. 


\section{Effect of the in vitro addition of LPS on the basal $V_{\mathrm{ms}}$ of isolated, perfused trachea from naive animals}

Because this study has shown that in vivo LPS-treatment ( $4 \mathrm{mg} / \mathrm{kg}$, i.p; $18 \mathrm{hr}$ posttreatment) significantly hyperpolarized the basal $\mathrm{V}_{\mathrm{ms}}$ (Figure 26), it was of interest to investigate whether or not the in vitro application of LPS to the organ bath did the same. The addition of LPS $(10 \mu \mathrm{g} / \mathrm{ml})$ to the EL and IL surfaces of isolated, perfused trachea from naive guinea pigs caused a nearly significant $(p=0.055)$ hyperpolarization of the basal $\mathrm{V}_{\mathrm{ms}}$ when compared to the control preparation to which nothing was added (Figure 41). In the untreated, control preparation, the reported $\triangle V_{\mathrm{ms}}$ was due to a slight hyperpolarization of the $\mathrm{V}_{\mathrm{ms}}$ over time which is inherent to the tissue. In addition, LPS caused a significant change in the basal $\mathrm{V}_{\mathrm{ms}}$ when compared to the $\triangle V_{\mathrm{ms}}$ in the absence of LPS.

\section{Effect of LPS-treatment on in vitro airway reactivity to MCh in epithelium- intact and -denuded isolated, perfused trachea}

Previous studies have shown that increases and decreases in EpDRF-induced smooth muscle relaxation were associated with alterations in in vitro and in vivo airway reactivity to MCh (Lawrence et al., 1996; Fedan et al., 2000). In this study LPS-treatment was shown to alter EpDRF-induced smooth muscle relaxation; therefore, it is hypothesized that in vitro and/or in vivo airway reactivity to $\mathrm{MCh}$ should be altered in accordance with the aforementioned studies. 

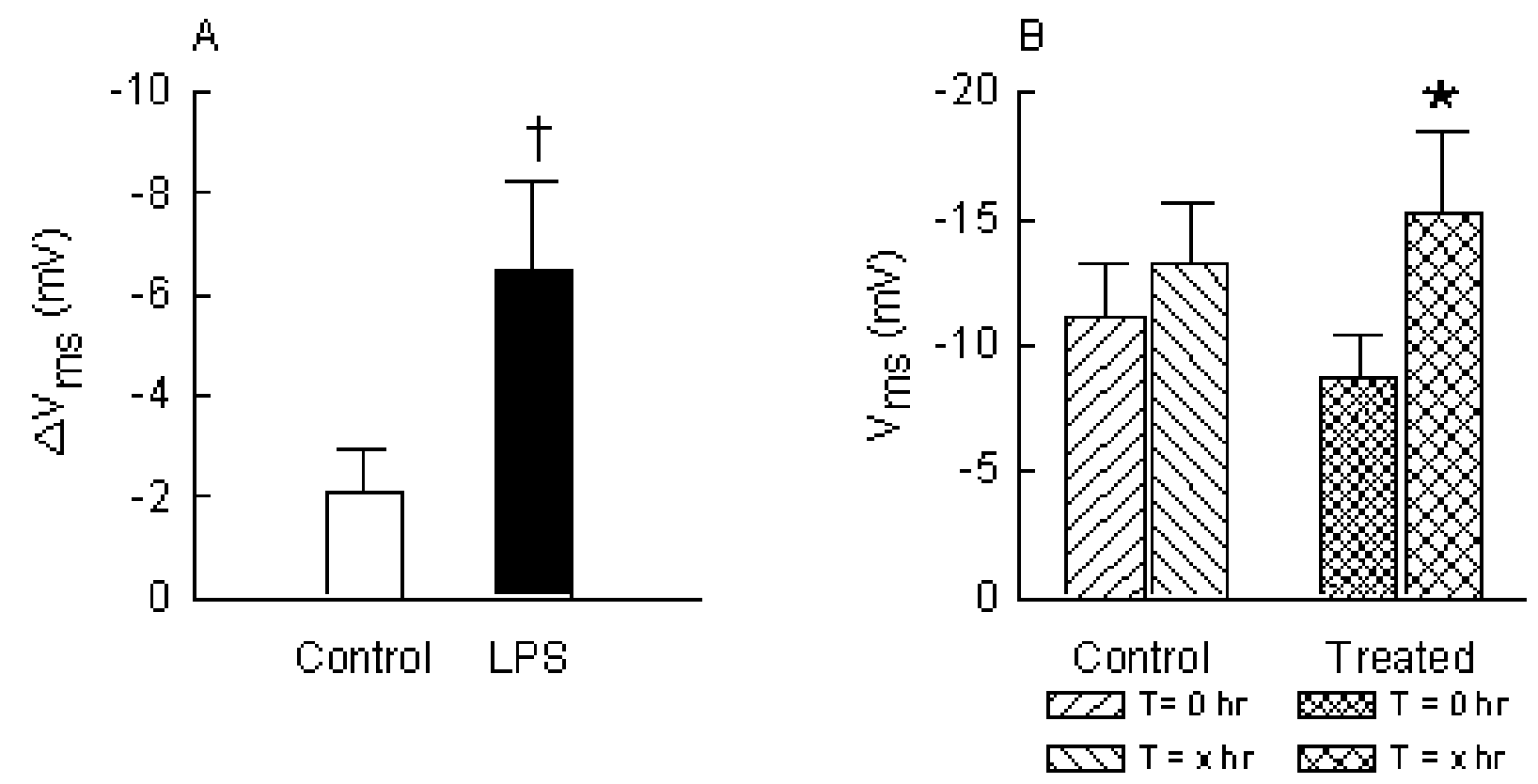

Figure 41. Effect of the in vitro addition of LPS on the basal $\mathrm{V}_{\mathrm{ms}}$ of isolated, perfused trachea from naive animals. Following a $3 \mathrm{hr}$ equilibration period, LPS $(10 \mu \mathrm{g} / \mathrm{ml})$ was simultaneously added to the EL and IL baths. (A) The hyperpolarizing effect of LPS on the basal $\mathrm{V}_{\mathrm{ms}}$ reached a nearly significant level when compared to the untreated, control preparation. The reported $\triangle V_{\text {ms }}$ for the untreated, control preparation was due to a slight hyperpolarization of the $\mathrm{V}_{\mathrm{ms}}$ over time which was inherent to the tissue. (B) There was a significant difference between the $V_{\mathrm{ms}}$ ' $\mathrm{s}$ before and after the addition of LPS. $\mathrm{t}=0 \mathrm{hr}$ refers to the time immediately before the start of the control- or LPS-treatment period. $\mathrm{t}=\mathrm{x}$ hr refers to the length of the control- or LPStreatment period which was dependent upon the time required for the response to LPS to equilibrate, and the length of this period was variable between tracheas. Control- and LPStreated, $n=5$. $\dagger p=0.055$ compared to control-treated as determined by a nonpaired Student's $t$ test. ${ }^{*} p<0.05$ compared to ! LPS as determined by a paired Student's $t$ test. 
In epithelium-intact tracheas, there was no difference in reactivity to extraluminally- and intraluminally-applied MCh between the two treatment groups; however, the tracheas from both treatment groups were more sensitive to extraluminally-applied MCh (Figure 42A, Table 4). In addition, there was no difference in the IL/EL maximum response ratio between the saline- and LPS-treated groups.

In epithelium-denuded tracheas, the differences between EL and IL reactivity to MCh were abolished, and the IL/EL maximum response ratio approached unity (Figure 42B, Table 5). In addition, there was no difference in reactivity to extraluminally- and intraluminally-applied MCh in epithelium-denuded tracheas from saline- and LPS-treated animals.

\section{Effect of LPS-treatment on tracheal epithelial bioelectric reactivity to MCh in isolated, perfused trachea}

Although LPS-treatment did not affect in vitro smooth muscle reactivity to MCh in epithelium-intact and -denuded isolated, perfused tracheas, it had a profound effect upon tracheal epithelial bioelectric reactivity to $\mathrm{MCh}$.

In tracheas from both saline- and LPS-treated animals, extraluminally-applied MCh produced a biphasic concentration-response curve (Figure 43 and Table 6). At MCh concentrations less than $10^{! 6} \mathrm{M}$, the $\mathrm{V}_{\mathrm{ms}}$ hyperpolarized while at $\mathrm{MCh}$ concentrations greater than $10^{! 6} \mathrm{M}$, the $\mathrm{V}_{\mathrm{ms}}$ depolarized. The $\mathrm{EC}_{50}{ }^{\prime} \mathrm{s}$ and maximum responses for hyperpolarization were smaller and larger, respectively, in the LPS-treated group; however, the $\mathrm{MCh}^{\mathrm{E}} \mathrm{C}_{50}$ 's and maximum responses for depolarization were not different. The IL application of MCh also generated biphasic electrical concentration-response curves in both treatment groups (hyperpolarizing at MCh concentrations less than $10^{! 3} \mathrm{M}$ and depolarizing at $\mathrm{MCh}$ concentrations greater than $10^{! 3} \mathrm{M}$; Figure 43 and Table 6). 


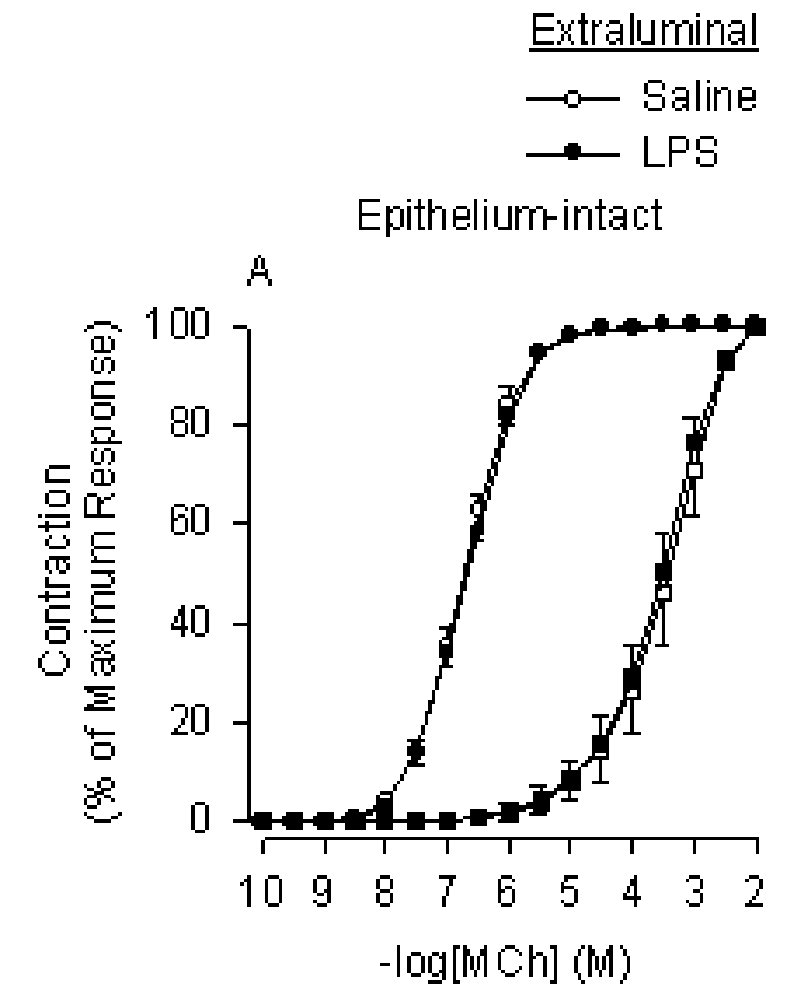

$$
\begin{aligned}
& \frac{\text { Intraluminal }}{\longrightarrow-\text { Saline }} \\
& \rightarrow-\text { LPS }
\end{aligned}
$$

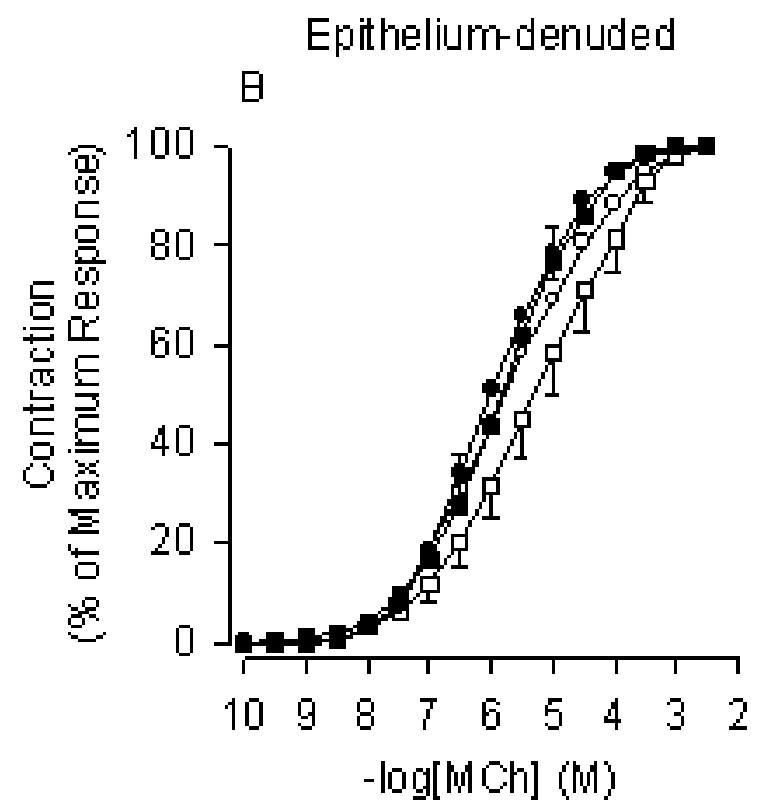

Figure 42. Lack of effect of LPS-treatment on tracheal smooth muscle reactivity to extraluminally- and intraluminally-applied MCh in (A) epithelium-intact and (B) -denuded isolated, perfused trachea. EL and IL MCh concentration-response curves were obtained from tracheas removed from saline- and LPS-treated animals $18 \mathrm{hr}$ post-treatment. In epitheliumintact tracheas from saline- and LPS-treated animals, extraluminally-applied MCh was more potent. Removal of the epithelium abolished the difference between reactivity to extraluminallyand intraluminally-applied MCh. Saline- and LPS-treated, $n=6$ for epithelium-intact and $n=4$ for epithelium-denuded. 
TABLE 4

Effect of LPS-treatment on in vitro airway reactivity to

MCh in epithelium-intact, isolated, perfused trachea

\begin{tabular}{|c|c|c|c|c|c|}
\hline \multirow[b]{2}{*}{ Treatment $(n)$} & \multicolumn{2}{|c|}{$\begin{array}{l}\mathrm{EC}_{50}(\mathrm{M}) \\
(95 \% \text { C.I. })\end{array}$} & \multicolumn{2}{|c|}{$\begin{array}{c}\text { Maximum Response } \\
\triangle \mathrm{P}\left(\mathrm{cm} \mathrm{H}_{2} \mathrm{O}\right)\end{array}$} & \multirow[t]{2}{*}{$\begin{array}{l}\text { IL/EL Maximum } \\
\text { Response Ratio }^{a}\end{array}$} \\
\hline & EL & IL & EL & $\mathrm{IL}$ & \\
\hline Saline (6) & $\begin{array}{l}1.9 \times 10^{! 7} \\
(1.4-2.5)\end{array}$ & $\begin{array}{l}3.7 \times 10^{! 4 b} \\
(1.5-9.1)\end{array}$ & $10.7 \pm 1.5$ & $8.4 \pm 1.1^{c}$ & $0.78 \pm 0.03$ \\
\hline LPS (6) & $\begin{array}{l}2.1 \times 10^{! 7} \\
(1.7-2.4) \\
\end{array}$ & $\begin{array}{l}3.2 \times 10^{!} 4 d \\
(1.8-5.8) \\
\end{array}$ & $6.6 \pm 0.9$ & $4.7 \pm 0.9^{e}$ & $0.70 \pm 0.12$ \\
\hline
\end{tabular}

EL, extraluminal; IL, intraluminal. The tracheas were removed from the animals $18 \mathrm{hr}$ posttreatment. ${ }^{a}$ The IL/EL maximum response ratio is used to gauge the modulatory effect of the epithelium on the EL and IL maximum contractile responses. In the absence of the epithelium, the ratio is unity while it becomes less than unity in the presence of the epithelium. As the modulatory effect of the epithelium becomes more inhibitory, the ratio becomes smaller (Fedan and Frazer, 1992; Fedan et al., 2000). ${ }^{b}$ Significantly greater than saline (EL). ${ }^{c}$ Significantly less than saline (EL). ${ }^{d}$ Significantly greater than LPS (EL). ${ }^{e}$ Significantly less than LPS (EL). 
TABLE 5

Effect of LPS-treatment on in vitro airway reactivity to

MCh in epithelium-denuded, isolated, perfused trachea

\begin{tabular}{|c|c|c|c|c|c|}
\hline \multirow[b]{2}{*}{ Treatment $(n)$} & \multicolumn{2}{|c|}{$\begin{array}{l}\mathrm{EC}_{50}(\mathrm{M}) \\
(95 \% \text { C.I. })\end{array}$} & \multicolumn{2}{|c|}{$\begin{array}{l}\text { Maximum Response } \\
\triangle \triangle \mathrm{P}\left(\mathrm{cm} \mathrm{H}_{2} \mathrm{O}\right)\end{array}$} & \multirow[t]{2}{*}{$\begin{array}{l}\text { IL/EL Maximum } \\
\text { Response Ratio }^{a}\end{array}$} \\
\hline & EL & $\mathrm{IL}$ & EL & $\mathrm{IL}$ & \\
\hline Saline (4) & $\begin{array}{l}2.1 \times 10^{! 6} \\
(0.8-5.2)\end{array}$ & $\begin{array}{l}7.7 \times 10^{! 6} \\
(2.0-30.0)\end{array}$ & $3.8 \pm 0.7$ & $3.5 \pm 0.6$ & $0.97 \pm 0.12$ \\
\hline LPS (4) & $\begin{array}{l}1.1 \times 10^{!} 6 \\
(0.5-2.2)\end{array}$ & $\begin{array}{l}1.5 \times 10^{! 6} \\
(0.8-2.8)\end{array}$ & $3.9 \pm 0.4$ & $3.5 \pm 0.3$ & $1.00 \pm 0.06$ \\
\hline
\end{tabular}

EL, extraluminal; IL, intraluminal. The tracheas were removed from the animals $18 \mathrm{hr}$ posttreatment. ${ }^{a}$ The IL/EL maximum response ratio is used to gauge the modulatory effect of the epithelium on the EL and IL maximum contractile responses. In the absence of the epithelium, the ratio is unity while it becomes less than unity in the presence of the epithelium. As the modulatory effect of the epithelium becomes more inhibitory, the ratio becomes smaller (Fedan and Frazer, 1992; Fedan et al., 2000). 
In the case of intraluminally-applied MCh, the maximum hyperpolarization response was greater in the LPS-treated group with respect to the saline-treated controls while there was no difference in the depolarization responses among the two treatment groups.

\section{Effect of LPS-treatment on in vivo pulmonary function and airway reactivity to MCh}

Besides investigating whether or not LPS-treatment altered in vitro airway reactivity to MCh in the isolated, perfused trachea, this study also investigated whether or not LPS-treatment altered in vivo airway reactivity to inhaled, MCh aerosol.

The post-treatment saline BF was slightly, but significantly, less than the pre-treatment value; however, there were no other differences between the pre- or post-treatment SRaw or BF values within each treatment group (Figure 44).

Eighteen hr following saline-treatment, the animals' reactivity to inhaled, $\mathrm{MCh}$ aerosol

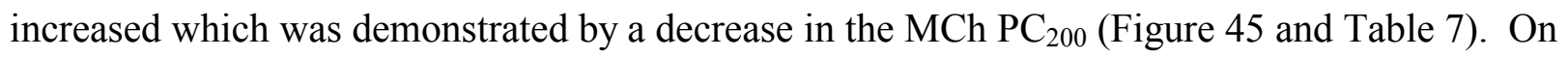
the other hand, LPS-treatment failed to produce any changes in airway reactivity to MCh when the LPS pre-and post-treatment groups were compared; however, when comparing the saline and LPS post-treatment groups to allow for the increase in reactivity in the saline-treated group, the LPS-treated animals were 3-fold hyporeactive to the effects of inhaled MCh as determined by a comparison of their $\mathrm{MCh}^{\mathrm{PC}_{200}}$ values (Table 7). 


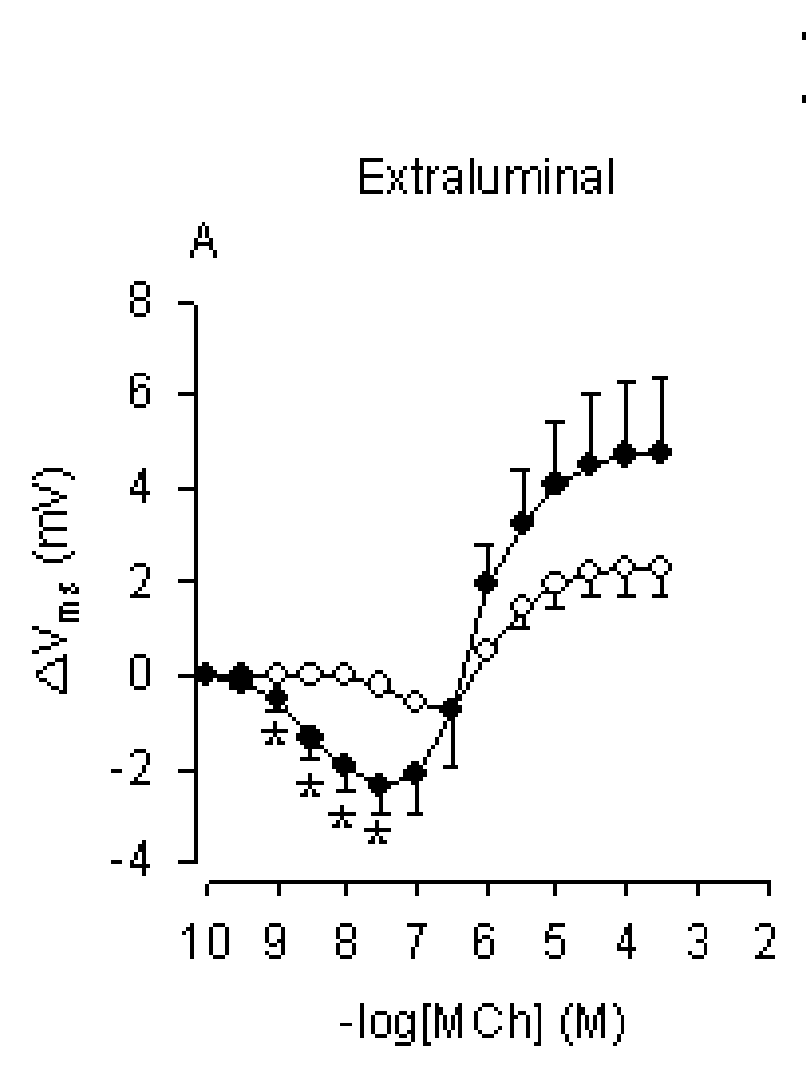

- Saline
LPS

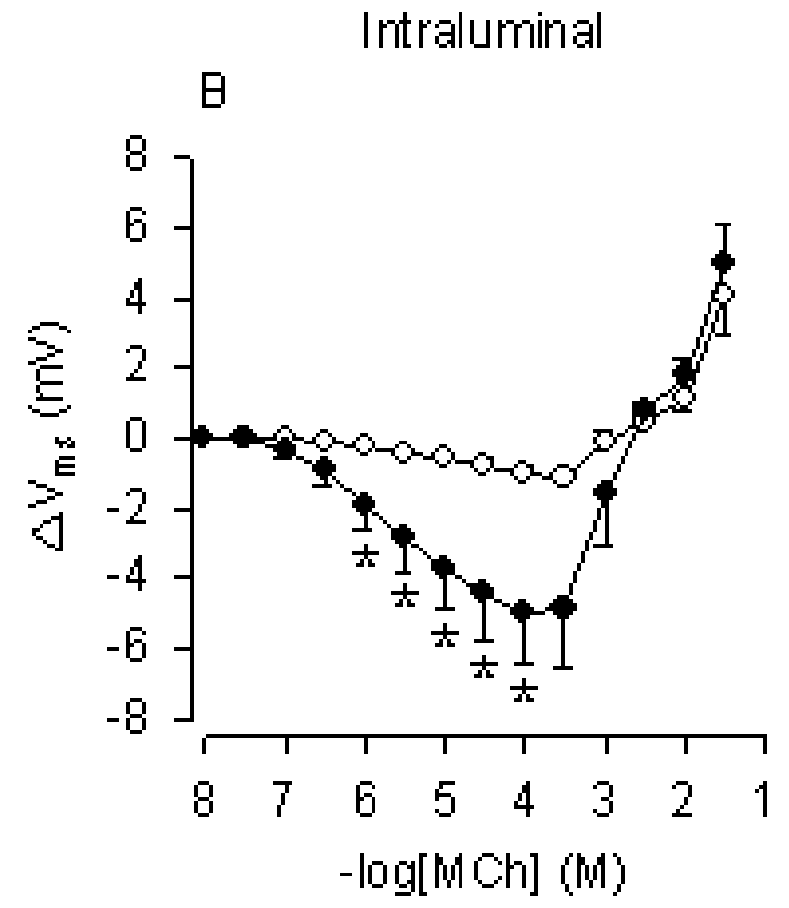

Figure 43. Effect of LPS-treatment on epithelial bioelectric reactivity to (A) extraluminally- and (B) intraluminally-applied MCh in isolated, perfused trachea. EL and IL MCh concentrationresponse curves were obtained from tracheas removed from saline- and LPS-treated animals 18 hr post-treatment. The results were expressed as changes in the transepithelial potential difference $\left(\triangle V_{m s}\right)$. Positive values refer to depolarization of the $V_{m s}$ while negative values refer to hyperpolarization of the $\mathrm{V}_{\mathrm{ms}}$. In both groups, extraluminally-applied $\mathrm{MCh}$ was more potent. Saline-treated, $n=7$ and LPS-treated, $n=6 .{ }^{*} p<0.05$ compared to saline-treated controls as determined by a nonpaired Student's $t$ test. 
TABLE 6

Effect of LPS-treatment on in vitro epithelial bioelectric reactivity to

MCh in isolated, perfused trachea

\begin{tabular}{|c|c|c|c|c|}
\hline \multirow[b]{2}{*}{ Treatment $(n)$} & \multicolumn{2}{|c|}{$\begin{array}{l}\mathrm{EC}_{50}(\mathrm{M}) \\
(95 \% \text { C.I. })\end{array}$} & \multicolumn{2}{|c|}{$\begin{array}{c}\text { Maximum Response } \\
\triangle \mathrm{V}_{\mathrm{ms}}(\mathrm{mV})^{a}\end{array}$} \\
\hline & EL & IL & EL & IL \\
\hline \multicolumn{5}{|c|}{ Hyperpolarization } \\
\hline Saline (7) & $\begin{array}{l}6.2 \times 10^{!} ! 8 \\
(2.9-13.0)\end{array}$ & $\begin{array}{l}9.8 \times 10^{!} 6 b \\
(2.1-45.0)\end{array}$ & ! $0.9 \pm 0.3$ & ! $1.6 \pm 0.3$ \\
\hline LPS (6) & $\begin{array}{l}4.2 \times 10^{! 9 c} \\
(1.6-11.0)\end{array}$ & $\begin{array}{c}2.7 \times 10^{!} 6 d \\
(1.8-5.9)\end{array}$ & ! $3.0 \pm 0.7^{b}$ & ! $5.3 \pm 1.6^{\epsilon}$ \\
\hline \multicolumn{5}{|l|}{ Depolarization } \\
\hline Saline (7) & $\begin{array}{l}2.6 \times 10^{!} 6 \\
(1.5-4.3)\end{array}$ & $\begin{array}{l}7.6 \times 10^{!} 2 b \\
(2.3-23.0)\end{array}$ & $2.3 \pm 0.6$ & $4.0 \pm 1.1$ \\
\hline LPS (6) & $\begin{array}{l}1.9 \times 10^{!} 6 \\
(1.0-3.7)\end{array}$ & $\begin{array}{l}2.8 \times 10^{! 2 d} \\
(0.7-10.0)\end{array}$ & $4.8 \pm 1.6$ & $5.0 \pm 1.1$ \\
\hline
\end{tabular}

EL, extraluminal; IL, intraluminal. The tracheas were removed from the animals $18 \mathrm{hr}$ posttreatment. ${ }^{a}$ Values refer to the change in the $\mathrm{V}_{\mathrm{ms}}{ }^{b}$ Significantly greater than saline (EL). ${ }^{c}$ Significantly less than saline (EL). ${ }^{d}$ Significantly greater than LPS (EL). ${ }^{e}$ Significantly greater than saline (IL). 

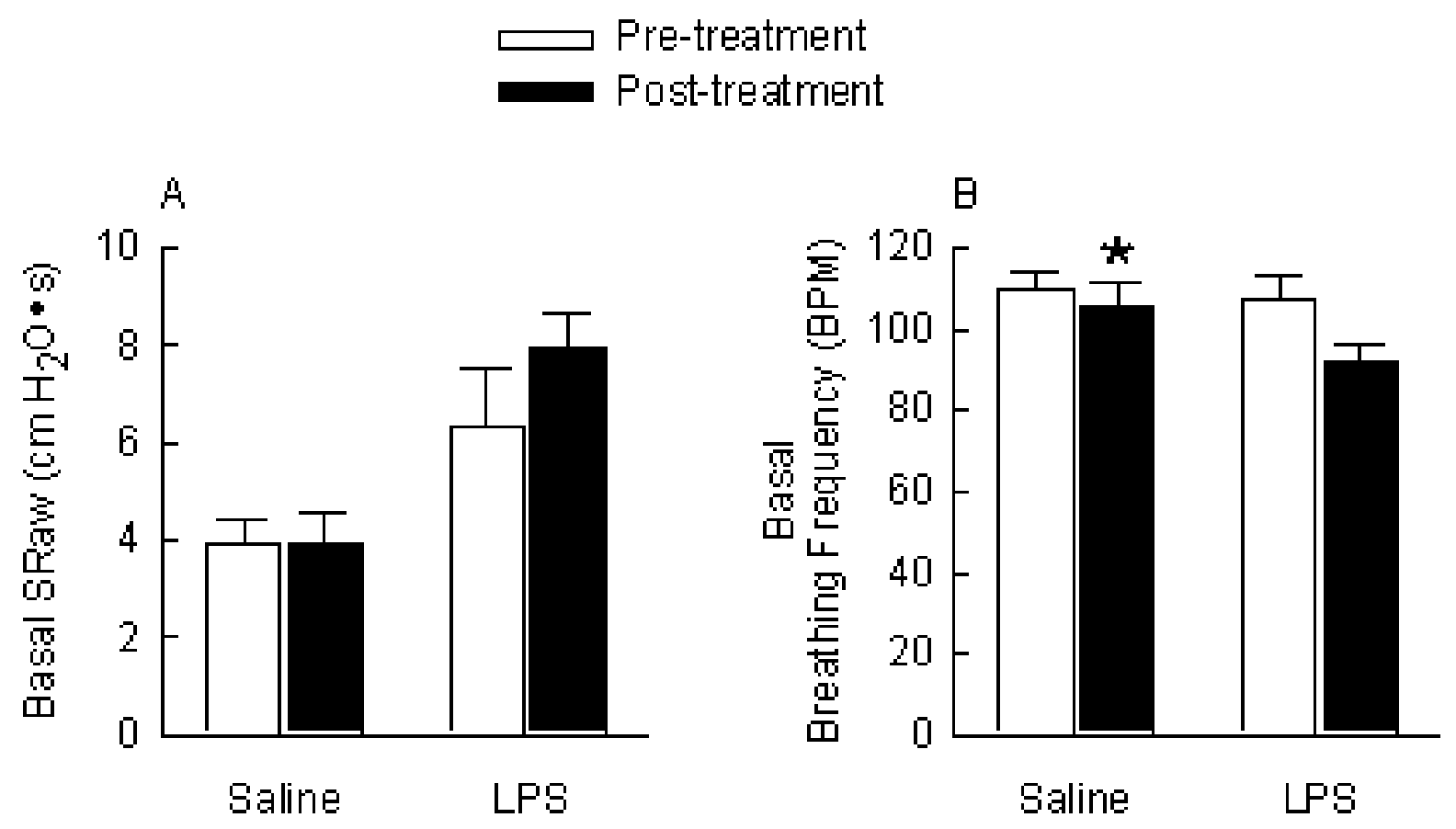

Figure 44. Effect of saline- and LPS-treatments on post-treatment basal (A) specific airway resistance (SRaw) and (B) breathing frequency (BF). Eighteen hr following saline- and LPStreatments, basal SRaw and BF were measured. There was a slight, but significant, decrease in the saline post-treatment BF when compared to the pre-treatment value. There were no other differences between the pre- and post-treatment values. Saline- and LPS-treated, $n=6$. $*_{p}<$ 0.05 compared to saline pre-treatment as determined by a paired Student's $t$ test. 


\section{o Pretreatment \\ - Post-treatment}
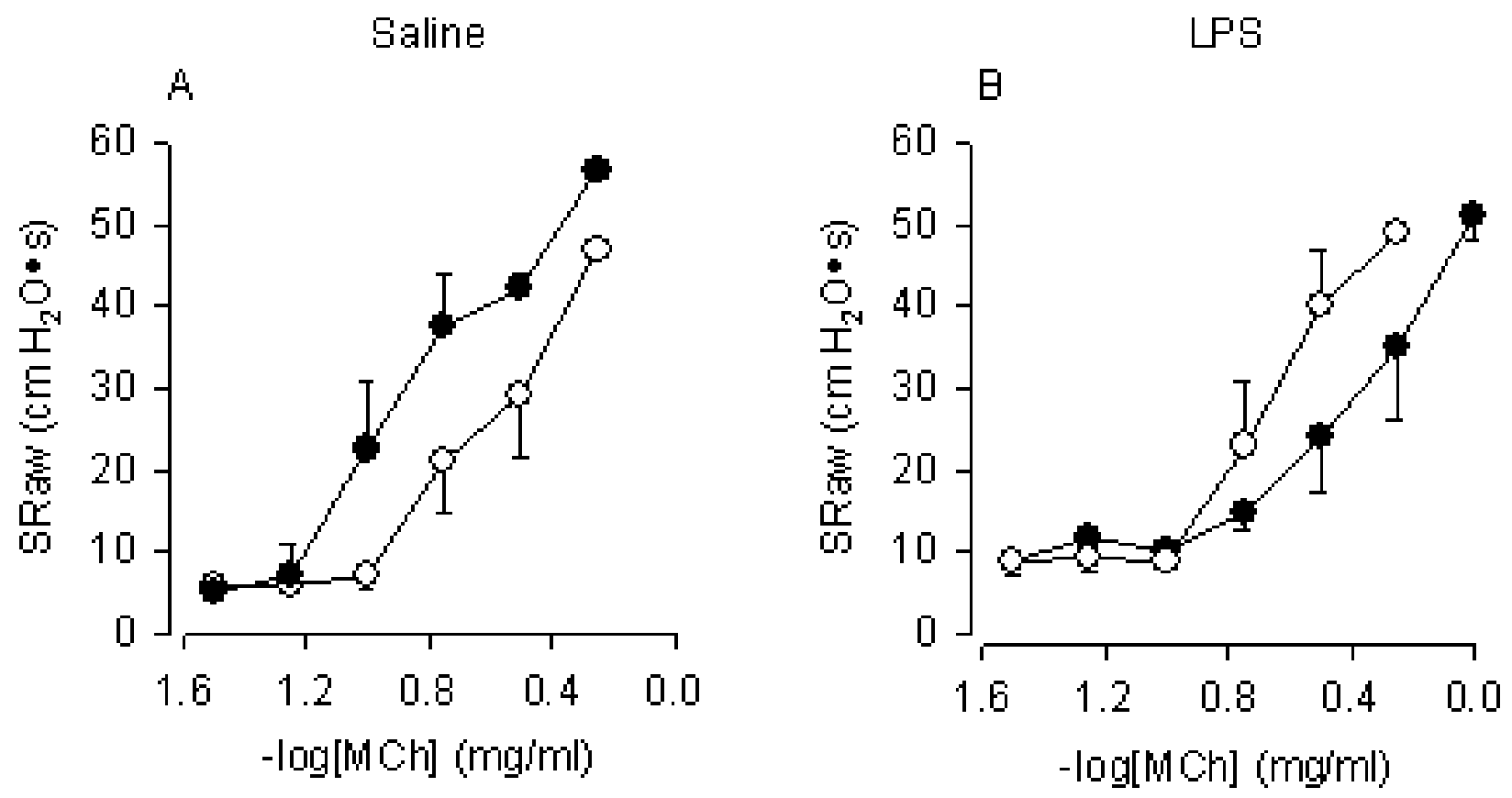

Figure 45. Effect of (A) saline- and (B) LPS-treatments on in vivo airway reactivity to inhaled $\mathrm{MCh}$ aerosol. Eighteen hr post-treatment, (A) the reactivity of the saline-treated animals to inhaled, MCh aerosol increased while (B) LPS-treatment had no significant effect on airway reactivity. When comparing the saline- and LPS-treated groups to allow for the increase in reactivity of the saline-treated group, the LPS-treated animals were hyporeactive to the effects of inhaled MCh. Saline- and LPS-treated, $n=6$. 
TABLE 7

\section{Effect of saline- and LPS-treatments on in vivo airway}

reactivity to inhaled MCh

\begin{tabular}{ccc}
\hline & \multicolumn{3}{c}{$\begin{array}{c}\text { MCh } \mathrm{PC}_{200}(\mathrm{mg} / \mathrm{ml}) \\
(95 \% \text { C.I. })\end{array}$} \\
\cline { 2 - 4 } Treatment $(n)$ & Pre-treatment & Post-treatment \\
\hline Saline (6) & 0.17 & $0.08^{a}$ \\
& $(0.11-0.27)$ & $(0.05-0.14)$ \\
LPS (6) & 0.18 & $0.26^{b}$ \\
& $(0.13-0.25)$ & $(0.08-0.79)$ \\
\hline
\end{tabular}

${ }^{a}$ Significantly less than saline (pre-treatment). ${ }^{b}$ Significantly greater than saline (post-treatment). 


\section{Effect of L-NAME on in vivo airway reactivity to MCh in saline- and LPS- treated animals}

In order to determine if an acquired NO component contributed to the in vivo airway hyporeactivity of the LPS-treated animals, the effect of an L-NAME aerosol on in vivo airway reactivity to MCh was determined in saline-and LPS-treated animals. Prior to the generation of in vivo MCh concentration-response curves in the presence of L-NAME, the LPS-treated animals were again found to be hyporeactive to the effects of inhaled MCh as demonstrated by a comparison of $\mathrm{MCh} \mathrm{PC}_{200}$ values from saline- and LPS-treated animals (Figure 46 and Table 8). The administration of L-NAME prior to MCh had no effect upon airway reactivity in either the saline- or LPS-treated animals.

\section{Histological Examination of Guinea-Pig Airways Following LPS-Treatment}

Since L-NAME failed to provide any clues to the mechanism underlying LPS-induced in vivo airway hyporeactivity to MCh, this study then examined the effects of LPS-treatment on the microscopic structure of various regions of the guinea-pig respiratory tract. This was done to determine if LPS-treatment altered the physical structure of the airways in such a way as to limit access of MCh to the airway smooth muscle (i.e., excessive mucus secretion, epithelial and basement membrane hyperplasia and/or hypertrophy, etc.). Histological sections of the distal trachea as well as the azygous, left, right apical, right cardiac, and right diaphragmatic lobes of the lung from saline- and LPS-treated animals were examined. The sections from both treatment groups were indistinguishable from one another, at least at the level of the light microscope. 


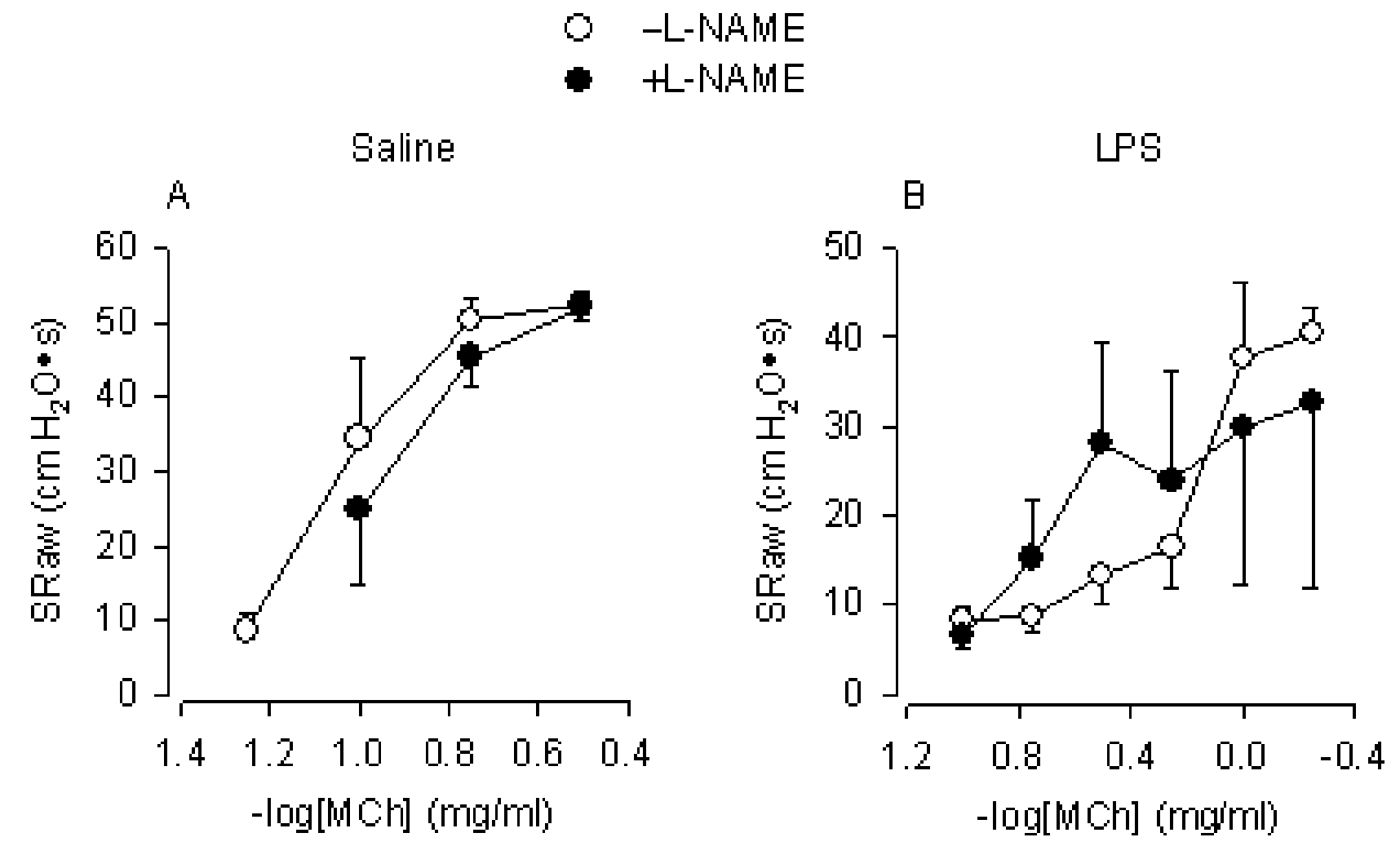

Figure 46. Lack of effect of L-NAME on in vivo airway reactivity to inhaled MCh aerosol in (A) saline- and (B) LPS-treated guinea pigs. Eighteen hr post-treatment, L-NAME did not alter airway reactivity to $\mathrm{MCh}$ in either treatment group. Saline- and LPS-treated, $n=4$. 
TABLE 8

Effect of L-NAME on in vivo airway reactivity to inhaled MCh in saline- and LPS-treated guinea pigs

\begin{tabular}{ccc}
\hline & \multicolumn{3}{c}{$\begin{array}{c}\text { MCh } \text { PC }_{200}(\mathrm{mg} / \mathrm{ml}) \\
(95 \% \text { C.I. })\end{array}$} \\
\cline { 2 - 4 } Treatment $(n)$ & ! L-NAME & + L-NAME \\
\hline Saline (4) & 0.07 & 0.01 \\
& $(0.06-0.10)$ & $(0.00-0.94)$ \\
LPS (4) & $0.78^{a}$ & 0.40 \\
& $(0.31-1.97)$ & $(0.11-1.45)$ \\
\hline
\end{tabular}

${ }^{a}$ Significantly greater than saline (! L-NAME). 
Representative sections of the distal trachea and left lobe of the lung from saline- and LPStreated animals are shown in Figure 47A-F. The bronchial and tracheal epithelium as well as the alveolar sacs remained structurally intact within each group. Finally, in both treatment groups, a few inflammatory cells, namely eosinophils and neutrophils, could be seen infiltrating the airways in some regions of the respiratory tract which were examined (data not shown). 


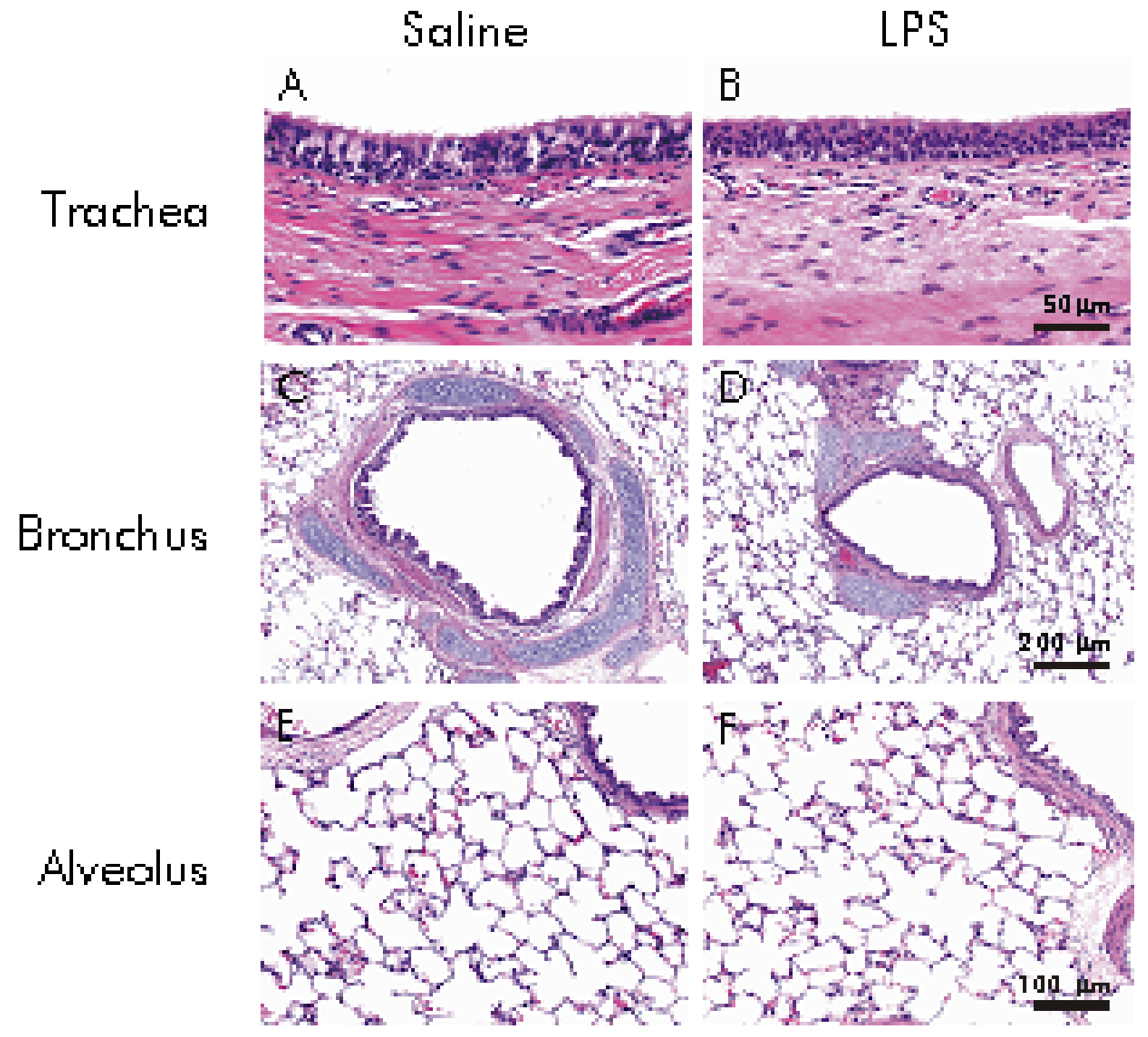

Figure 47. Histological sections of guinea-pig airways following saline- and LPS-treatments. Representative Harris' hematoxylin-eosin stained histological sections from saline (A, C, and E)and LPS (B, D, and F)-treated animals made $18 \mathrm{hr}$ post-treatment. A thorough examination of histological sections from saline- and LPS-treated animals showed that LPS-treatment did not alter airway appearance or structure, at least at the level of the light microscope. A and B are $40 \times$ sections of distal trachea, $\mathrm{C}$ and $\mathrm{D}$ are $10 \times$ sections of bronchi, and $\mathrm{E}$ and $\mathrm{F}$ are $20 \times$ sections of the alveolar regions from saline- and LPS-treated animals, respectively. Sections of the bronchi and alveolar regions were taken from the left lobe of the lung. The measurement bars $(50,200$, and $100 \mu \mathrm{m})$ found in $\mathrm{B}, \mathrm{D}$, and $\mathrm{F}$ also pertain to their respective counterpart in $\mathrm{A}, \mathrm{C}$, and $\mathrm{E}$. These results are representative of eight different experiments from each treatment group. 


\section{Discussion}

There are three general conclusions which can be drawn from the results of this study. First, the effects of EpDRF on the airway smooth muscle are associated with changes in the electrical activity of the airway epithelium following LPS-treatment; however, these changes in the effects of EpDRF are dependent upon the osmolyte used to elicit EpDRF release. Secondly, epithelial depolarization does not always precede EpDRF-induced smooth muscle relaxation, suggesting that EpDRF release is not always dependent on epithelial depolarization. Finally, LPS-induced in vivo airway hyporeactivity to inhaled, MCh aerosol may be due, at least in part, to an increase in the actions of EpDRF.

Both in vivo and in vitro LPS-treatment significantly hyperpolarized the basal $\mathrm{V}_{\mathrm{ms}}$ of isolated, perfused trachea. To our knowledge, there have been very few studies examining the effect of LPS on ion channel and/or transporter function; however, those studies which have examined this phenomenon aid in the explanation of our results. In rat tail artery smooth muscle cells, LPS increased the inward flux of $\mathrm{Ca}^{2+}$ ions through voltage-sensitive $\mathrm{Ca}^{2+}$ channels (Wilkinson et al., 1996) while two other, separate studies reported that LPS increased the conductance of $\mathrm{Ca}^{2+}$-activated $\mathrm{K}^{+}$channels in vascular smooth muscle cells (Miyoshi and Nakaya, 1994; Hoang et al., 1997). In our system, LPS or one of its mediators may be increasing epithelial cell $\mathrm{Ca}^{2+}$ influx which could activate $\mathrm{Ca}^{2+}$-activated $\mathrm{K}^{+}$channels found on the basolateral surface of the airway epithelium (Clarke and Boucher, 1993). It is intriguing to note that $\mathrm{Ca}^{2+}$-activated $\mathrm{K}^{+}$channels have been shown to be involved in the synthesis and/or release of EpDRF (Tamaoki et al., 1997), and their activation by LPS or one of its many mediators may account for the alterations in EpDRF-induced smooth muscle relaxation following LPS-treatment.

The alteration of EpDRF-induced smooth muscle relaxation following LPS-treatment was dependent upon the osmolyte used to elevate IL tonicity, suggesting that the synthesis, release, and/or effects of EpDRF are osmolyte-dependent, at least after LPS-treatment. It is 
difficult to reconcile these results with the fact that EpDRF-induced smooth muscle relaxation is initiated regardless of the osmolyte used to elevate EL or IL tonicity (Munakata et al., 1988; Fedan et al., 1999). One study has examined the potency of different osmolytes to elicit EpDRF-induced smooth muscle relaxation. This study reported that $\mathrm{NaCl}$ and $\mathrm{KCl}$ were equipotent in their ability to evoke EpDRF-induced smooth muscle relaxation; however, no other agents were examined (Fedan et al., 1999). The differences among the osmolytes used in the present study could be due to their ability or inability to interact with specific epithelial ion channels and/or transporters, namely apical $\mathrm{Na}^{+}$and $\mathrm{Cl}^{!}$channels, which seem to be involved in EpDRF-induced smooth muscle relaxation (Fedan et al., 1999). Dmannitol is membrane-impermeable so it would be unable to pass through any ion channel and/or transporter while urea is freely, membrane-permeable. On the other hand, both $\mathrm{NaCl}$ and $\mathrm{KCl}$ can enter the cell through their respective ion channels and/or transporters. In addition, this study showed that LPS-treatment altered the basal bioelectric properties of the epithelium which could further modify EpDRF-induced smooth muscle relaxation in response to these osmolytes if a specific ion channel and/or transporter involved in these relaxation responses were altered following LPS-treatment. It is of interest to note that a previous study examined the effects of $\mathrm{NaCl}$, mannitol, and urea on isolated, human bronchial strips, and they reported that hypertonic $\mathrm{NaCl}$ and mannitol initiated bronchoconstriction while urea did not (Jongejan et al., 1990). As a side note, the contractile responses elicited by these osmolytes may be attributed to species differences or more importantly to the fact that one cannot selectively add pharmacological agents to either the serosal or mucosal surfaces of strip preparations. Although our study looked at EpDRF-induced smooth muscle relaxation elicited by these agents, our study and that of Jongejan et al. (1990) demonstrated that hypertonic $\mathrm{NaCl}$ and $\mathrm{D}$-mannitol behave in a similar manner regardless of the endpoint, contraction or relaxation, examined. In our study, $\mathrm{NaCl}$ and D-mannitol seem to be working by a similar mechanism while urea and $\mathrm{KCl}$ act by a different mechanism from each other as well as from $\mathrm{NaCl}$ and D-mannitol following LPS-treatment. 
We investigated whether the potentiation of EpDRF-induced smooth muscle relaxation, elicited by $\mathrm{NaCl}$, following LPS-treatment was due to an acquired $\mathrm{NO}$ and/or prostanoid component because both $\mathrm{NO}$ and certain prostanoids are known smooth muscle relaxants (Folkerts and Nijkamp, 1998). In addition, LPS can up-regulate inducible nitric oxide synthase as well as potentiate the smooth muscle relaxant effects of some prostanoids in guinea pigs (Stein et al., 1996; Myers et al., 1999). Both L-NAME and indomethacin, inhibitors of nitric oxide synthase and cyclooxygenase, respectively, failed to have any effect upon EpDRF-induced smooth muscle relaxation in tracheas from saline- and LPS-treated animals. This further supports the conclusion that EpDRF is neither NO nor a prostanoid and that the potentiation of EpDRF-induced smooth muscle relaxation following LPS-treatment was not due to an acquired NO or prostanoid component.

The potentiation of EpDRF-induced smooth muscle relaxation following LPS-treatment was also associated with a potentiation of the epithelial bioelectric events, depolarization of the $\mathrm{V}_{\mathrm{ms}}$, preceding EpDRF-induced smooth muscle relaxation when $\mathrm{NaCl}$ was used to elevate $\mathrm{IL}$ tonicity. These results further strengthen the hypothesis that the two events are functionally linked. Both hypertonicity-induced smooth muscle relaxation and epithelial depolarization were potentiated following LPS-treatment; however, in both treatment groups, the epithelium more readily released EpDRF than depolarized in response to elevated mucosal tonicity. The epithelium can release EpDRF, as demonstrated by smooth muscle relaxation, when as little as $0.84 \mathrm{mOsm}$ of $\mathrm{NaCl}$ was added to the IL surface of the trachea; however, the $\mathrm{V}_{\mathrm{ms}}$ did not depolarize in response to hypertonicity until approximately $8.44 \mathrm{mOsm}$ of added $\mathrm{NaCl}$. If epithelial depolarization in response to hypertonicity signaled the release of EpDRF (Dortch-Carnes et al., 1999), we would have expected the epithelium to depolarize prior to every relaxation response; however, this was clearly not the case. Because of this discrepancy, it is tempting to speculate that two events are taking place. At low concentrations of hypertonicity, EpDRF release is independent of epithelial depolarization, a situation analogous to pharmacomechanical coupling. As EpDRF is released in response to higher concentrations of 
hypertonicity, epithelial depolarization may be a necessary component to signal the release of greater quantities of EpDRF, a situation analogous to electromechanical coupling.

Alternatively, transepithelial depolarization may be quite, simply a coincident, epiphenomenon occurring in parallel with another signal (i.e., change in intracellular $\mathrm{pH}$ ) that is more sensitive to osmolarity changes. It is quite possible that changes in intracellular $\mathrm{pH}$, and not transepithelial depolarization, in response to elevated mucosal or serosal tonicity is the signal for the synthesis, release, and/or effects of EpDRF. This is a potential avenue of further investigation given the fact that exposure of cells to hyperosmotic solution causes cell alkalinization (Miyata et al., 2000). In addition, the $\mathrm{Na}^{+}$channel blocker, amiloride, and the $\mathrm{Cl}^{\prime}$ channel blocker, DIDS, which attenuate EpDRF-induced smooth muscle relaxation, are also capable of inhibiting the $\mathrm{Na}^{+}-\mathrm{H}^{+}$exchanger and the $\mathrm{Cl}^{-}-\mathrm{HCO}_{3}{ }^{!}$exchanger (Kreydiyyeh, 2000; Lamb et al., 2000); respectively. The actions of these pharmacological inhibitors on their respective targets would, without a doubt, profoundly influence intracellular $\mathrm{pH}$.

This study also examined epithelial bioelectric reactivity to hypertonic $\mathrm{NaCl}$ in tracheas from saline- and LPS-treated animals. These experiments were done in the absence of MCh since smooth muscle contraction was not a necessary component of these experiments. In both treatment groups, the epithelium depolarized in response to extraluminally- and intraluminallyapplied $\mathrm{NaCl}$ with the EL surface of the trachea being more sensitive to hypertonicity. This was in contrast to another study examining the effect of hypertonicity on airway epithelial bioelectric responses, in which cultured, human nasal epithelial cells depolarized only when hypertonic mannitol was added to the apical surface of the epithelium (Willumsen et al., 1994). This discrepancy may be attributed to species or regional differences in ion transport (Boucher et al., 1980). Tracheas from LPS-treated animals were also less sensitive to the effects of intraluminally-applied $\mathrm{NaCl}$ than those from saline-treated animals. Because the basal $\mathrm{V}_{\mathrm{ms}}$ in tracheas from LPS-treated animals was significantly more hyperpolarized, it may be more difficult to depolarize the $\mathrm{V}_{\mathrm{ms}}$ or the ion channels and/or transporters affected by hypertonicity could be less sensitive to the effect of IL hypertonicity following LPS-treatment. Overall, these 
results demonstrate that the EL and IL surfaces of the trachea can function as an osmotic sensor, with the EL surface being more sensitive. In addition, LPS-treatment alters the ability of the IL surface to act as an osmotic sensor.

This study investigated the mechanism(s) behind the hyperpolarization of the $\mathrm{V}_{\mathrm{ms}}$ following LPS-treatment by examining the effect of various pharmacological agents on the $\mathrm{V}_{\mathrm{ms}}$. Because LPS is known to up-regulate inducible nitric oxide synthase and cyclooxygenase, the effect of their inhibitors on the basal $\mathrm{V}_{\mathrm{ms}}$ was examined. The small hyperpolarization of the $\mathrm{V}_{\mathrm{ms}}$ by L-NAME in both treatment groups was not consistent with the reported effects of NO on ion transport. In rat colon and human airway epithelial cells, $\mathrm{NO}$ stimulated $\mathrm{Cl}^{\prime}$ secretion (Tamai and Gaginella, 1993; Kamosinska et al., 1997). If NO stimulates $\mathrm{Cl}^{!}$secretion in our system, L-NAME, by virtue of its ability to inhibit NO synthesis, should have depolarized the $\mathrm{V}_{\mathrm{ms}}$ which was clearly not the case. Sodium nitroprusside, a NO donor, depolarized the $\mathrm{V}_{\mathrm{ms}}$, which was not consistent with a previous report demonstrating its ability to stimulate $\mathrm{Cl}^{\text {! }}$ secretion (Tamai and Gaginella, 1993). Our results, however, were internally consistent. Since L-NAME hyperpolarized the $\mathrm{V}_{\mathrm{ms}}$ in our system, we would have expected a NO donor such as sodium nitroprusside to depolarize the $\mathrm{V}_{\mathrm{ms}}$. From both of these experiments, however, we can conclude that NO makes an insignificant contribution to the generation of the $\mathrm{V}_{\mathrm{ms}}$ in tracheas from either saline- or LPS-treated animals.

Because L-NAME has muscarinic antagonist properties (Buxton et al., 1993), we investigated the effects of atropine, a nonspecific muscarinic receptor antagonist, on the basal $\mathrm{V}_{\mathrm{ms}}$ in both treatment groups. The variable effects of atropine on the $\mathrm{V}_{\mathrm{ms}}$ observed in this study were not consistent with another study which reported that atropine profoundly and rapidly depolarized the $\mathrm{V}_{\mathrm{ms}}$ of rabbit trachea in vivo (Tamaoki et al., 1996). The variable effects of atropine seen in this study could have resulted from the antagonism of different muscarinic receptor subtypes, some of which may be more prevalent in one animal than another. This is a reasonable conclusion given the fact that this study has shown that at least two populations of muscarinic receptor subtypes exist in the guinea-pig tracheal epithelium which are capable of 
initiating opposite electrophysiological effects. The fact that atropine does have an electrophysiological effect suggests that the nerves endings embedded in the airway wall are tonically releasing $\mathrm{ACh}$, which exerts its effect by binding to epithelial muscarinic receptors. From our results with atropine, the true effect of L-NAME cannot be deciphered; however, a more rigorous, future study should use equieffective concentrations of atropine and L-NAME with respect to their muscarinic antagonist properties.

Unlike L-NAME, the electrophysiological effects of indomethacin reported in this study were consistent with other studies in the literature. Two prostaglandins in particular, $\mathrm{D}_{2}$ and $\mathrm{E}_{2}$, have been shown to stimulate $\mathrm{Cl}^{\text {! }}$ secretion in several unrelated species (Tamaoki et al., 1992a,b; Saunders et al., 1993; Bourke et al., 1995). Indomethacin depolarized the $\mathrm{V}_{\mathrm{ms}}$ in both treatment groups, but significantly more so in the LPS-treated group. The depolarizing effects of indomethacin were consistent with an inhibition of prostanoid-stimulated $\mathrm{Cl}^{!}$secretion. The fact that indomethacin depolarized the $\mathrm{V}_{\mathrm{ms}}$ to a greater extent in the LPStreated group suggests that prostanoids contribute more to the generation of the basal $\mathrm{V}_{\mathrm{ms}}$ following LPS-treatment and/or their effects were potentiated by changes in their receptor or post-receptor signaling. In addition, this suggests that prostanoid-stimulated $\mathrm{Cl}^{!}$secretion may contribute to the significant hyperpolarization of the $\mathrm{V}_{\mathrm{ms}}$ after LPS treatment.

The addition of amiloride to the IL surface of the trachea abolished the difference between the $V_{m s}$ 's among the two treatment groups. This strongly suggests that the hyperpolarization of the $\mathrm{V}_{\mathrm{ms}}$ following LPS-treatment was due to an increase in amiloridesensitive $\mathrm{Na}^{+}$transport or $\mathrm{Na}^{+}$transport in general. An increase in $\mathrm{Na}^{+}$removal from the lumen, via these channels, would generate a more negative $V_{m s}$. Because the airway epithelium consists of numerous ion channels and transporters, alterations in any of them (Figure 6) could explain changes in the $\mathrm{V}_{\mathrm{ms}}$ following LPS-treatment. For example, an increase in the number and/or activity of the $\mathrm{Na}^{+}-\mathrm{K}^{+}$-ATPase would increase amiloride-sensitive $\mathrm{Na}^{+}$transport and subsequently hyperpolarize the $\mathrm{V}_{\mathrm{ms}}$. More systematic experiments which would take advantage of patch clamping and transepithelial resistance and $I_{\mathrm{sc}}$ measurements, and other ion channel and 
transporter inhibitors would be necessary to determine the mechanism responsible for LPSinduced hyperpolarization of the $\mathrm{V}_{\mathrm{ms}}$. These results with amiloride also impact on the possible mechanism(s) underlying the potentiation of EpDRF-induced smooth muscle relaxation following LPS-treatment. If amiloride-sensitive $\mathrm{Na}^{+}$channels increase in number and/or activity following LPS-treatment, this may potentiate EpDRF-induced smooth muscle relaxation inasmuch as their inhibition significantly attenuates this response (Fedan et al., 1999).

The potentiation of EpDRF-induced smooth muscle relaxation following LPS-treatment was associated with in vivo airway hyporeactivity to inhaled, $\mathrm{MCh}$ aerosol while no differences in in vitro airway reactivity to MCh existed in isolated, perfused tracheas from saline- and LPStreated animals. Over the years, there have been several, inconsistent reports concerning the effect of LPS-treatment on in vitro and in vivo guinea-pig airway reactivity. In vivo, some studies have reported hyperreactivity (Oosterhout et al., 1991), hyporeactivity (Folkerts et al., 1988), and no change (Fedan et al., 1995) in guinea-pig airway reactivity to contractile agonists. The same can be said for in vitro airway reactivity as well. Again, some studies have reported hyperreactivity to contractile agonists (ACh, histamine, arecoline) (Folkerts et al., 1989; Yamawaki et al., 1990; Oosterhout et al., 1991), hyporeactivity (Folkerts et al., 1988), and finally, others have shown no change (Fedan et al., 1995) in in vitro guinea-pig airway reactivity. In our study, there were no differences in airway reactivity to MCh in epithelium-intact and -denuded isolated, perfused tracheas. This is consistent with a previous study in our laboratory (Fedan et al., 1995) even though the $18 \mathrm{hr}$ post-treatment time point was not examined. In vivo, LPS-treated guinea pigs were hyporeactive to the effects of inhaled, MCh aerosol. This is not consistent with the earlier, published reports from our laboratory, but again the $18 \mathrm{hr}$ post-treatment time point was not examined which may account for the difference (Fedan et al., 1995). An acquired NO component does not seem to be responsible for the hyporeactivity to MCh following LPS-treatment in our study since inhaled, L-NAME aerosol was without effect on in vivo airway reactivity to MCh in LPS-treated animals. 
In the previous study reporting in vivo airway hyporeactivity to histamine following LPStreatment (Folkerts et al., 1988), the airways of these guinea pigs were infiltrated with large numbers of neutrophils and monocytes; however, there were no signs of pulmonary inflammation following LPS-treatment in our study. This was probably due to the fact that the guinea pigs in the previous study were exposed to LPS by inhalation and examined $24 \mathrm{hr}$ posttreatment while our animals were given LPS i.p. and examined $18 \mathrm{hr}$ post-treatment. The lack of pulmonary inflammation in our LPS-treated guinea pigs suggests that changes in airway reactivity are not necessarily accompanied by airway inflammation. The airway hyporeactivity following LPS-treatment does not seem to be due to an acquired NO component or alterations in the physical structure of the airways but may be due, at least in part, to an increase in the actions of EpDRF. In vivo changes in airway reactivity did not manifest themselves in vitro which suggests that the mechanism responsible for in vivo hyporeactivity to MCh did not involve the trachea or was lost in vitro.

This is not the first time that alterations in the actions of EpDRF have been accompanied by changes in in vivo and/or in vitro airway reactivity. For example, exposure to ozone causes a decrease in the actions of EpDRF which is associated with in vivo and in vitro airway hyperreactivity to MCh (Fedan et al., 2000). In addition, sensitization and challenge of guinea pigs with ovalbumin potentiates EpDRF-induced smooth muscle relaxation which is accompanied by in vitro airway hyporeactivity to $\mathrm{MCh}$, but paradoxically, these animals are hyperreactive to MCh in vivo (Lawrence et al., 1996). Now, with the addition of our study, there are at least three instances in which changes in the actions of EpDRF are associated with in vivo and/or in vitro alterations in airway reactivity to $\mathrm{MCh}$, suggesting a functional relationship between the two.

Although LPS-treatment did not have an effect upon in vitro airway smooth muscle reactivity to $\mathrm{MCh}$, it did have a profound effect upon in vitro epithelial bioelectric reactivity to MCh. Biphasic concentration-response curves were generated in response to the EL- and ILapplication of $\mathrm{MCh}$ in both treatment groups. Our results were consistent with the variable 
electrophysiological effects produced by MCh in guinea-pig nasal epithelium (Suzuki et al., 1999). Suzuki et al. (1999) reported that MCh produced triphasic $I_{\mathrm{sc}}$ responses: a transient increase followed by a small decrease and then finally a sustained increased. The authors concluded that these changes were brought about by alterations in $\mathrm{Cl}^{\text {! }}$ secretion and amiloridesensitive $\mathrm{Na}^{+}$transport (Suzuki et al., 1999). $\mathrm{MCh}$, by binding to either, $\mathrm{M}_{1}, \mathrm{M}_{3}$, or $\mathrm{M}_{5}$ muscarinic receptors increases cytosolic $\mathrm{Ca}^{2+}$ by coupling $\mathrm{G}_{\mathrm{q}}$-proteins (Janssen et al., 1998). $\mathrm{Ca}^{2+}$ in turn is capable of activating $\mathrm{Ca}^{2+}$-activated $\mathrm{Cl}^{!}$channels and/or $\mathrm{Ca}^{2+}$-activated $\mathrm{K}^{+}$ channels (Somlyo and Somlyo, 1994) which could subsequently alter the $\mathrm{V}_{\mathrm{ms}}$. In addition, the activation of $\mathrm{M}_{2}$ and $\mathrm{M}_{4}$ muscarinic receptors can open nonselective cation channels and inhibit current flow through $\mathrm{Ca}^{2+}$ channels and $\mathrm{Ca}^{2+}$-activated $\mathrm{K}^{+}$channels by coupling $\mathrm{G}_{\mathrm{i}}$-coupled proteins (Roux et al., 1998). The activation of more than one channel was most likely responsible for these biphasic electrical responses, and it is not certain which muscarinic receptor subtypes were initiating these responses. $\mathrm{M}_{1}, \mathrm{M}_{2}$, and $\mathrm{M}_{3}$ muscarinic receptors have been localized in guinea-pig lung (Mak and Barnes, 1990), and in the future it may be useful to repeat these experiments in the presence of selective, muscarinic receptor antagonists. The muscarinic receptors which were mediating these responses seem to be localized to the basolateral surface of the epithelium since MCh was more potent when added to the EL surface of the trachea. Thus, there seem to be two types of muscarinic epithelial receptors which promote opposite electrical responses in the guinea-pig tracheal epithelium. The high affinity receptor initiates hyperpolarization while the low affinity receptor mediates depolarization, and LPS-treatment seems to selectively alter the function of the high affinity receptor.

Since LPS was used as a tool to alter the basal bioelectric properties of guinea-pig tracheal epithelium, the results of this study have implications with respect to certain pathophysiological conditions in which LPS is involved, such as sepsis, ARDS, as well as gramnegative pulmonary infections. Our study has shown that LPS-treatment alters the actions of EpDRF on the airway smooth muscle, and this may potentially be happening under a variety of LPS-induced disease states. At present, EpDRF's only known function is to relax the airway 
smooth muscle; however, it may be an important mediator of other unknown, physiological functions. That is, these other potential functions of EpDRF may be altered in such a way by LPS that it contributes to the disease process. EpDRF may, when present at high or low levels, be directly toxic to various cell types such that it inhibits their normal function or enhances the release of toxic substances from cells. Conversely, high or low levels of EpDRF may protect cells from the insulting effects of LPS in such a way as to prevent further damage.

In conclusion, the results of this study suggest that the effects of EpDRF on the airway smooth muscle are associated with changes in the electrical activity of the airway epithelium; however, there is not always a direct correlation between the two events. In addition, this study has shown that perturbations in the effects of EpDRF are associated with changes in airway reactivity, demonstrating that EpDRF exerts a powerful, modulatory influence on airway smooth muscle tone. 


\section{Conclusions}

The initial reports by Raeburn and Fedan (1989), Dortch-Carnes et al. (1999), and Fedan et al. (1999), suggesting that the synthesis, release, and/or effects of EpDRF were functionally linked to the electrical activity of the airway epithelium, have been confirmed by the further characterization of the airway epithelial bioelectric mechanisms associated with the effects of EpDRF in this study.

Fedan et al. (1999) previously reported that apical $\mathrm{Na}^{+}$channels are involved in hypertonicity-induced smooth muscle relaxation via EpDRF, and this study has shown that these channels are also involved in the epithelial bioelectric response to elevated mucosal tonicity. These data suggest that the mechanical and bioelectric events are functionally linked; however, the exact role of these channels in mediating these responses is unclear. Despite the fact that inhibitors of basolateral $\mathrm{Na}^{+}-\mathrm{K}^{+}-2 \mathrm{Cl}^{!}$cotransporters, basolateral $\mathrm{Na}^{+}-\mathrm{K}^{+}$-ATPases, and apical $\mathrm{Cl}^{!}$ channels altered the response to elevated mucosal tonicity, their effects were different, in some respects, from their actions on EpDRF-induced smooth muscle relaxation, which makes their overall role in these two responses difficult to discern.

This study has also demonstrated that perturbations in the basal electrical activity of the epithelium following LPS-treatment are associated with alterations in EpDRF-induced smooth muscle relaxation and its associated bioelectric events as well as airway reactivity. This further supports the notion that the synthesis, release, and/or effects of EpDRF are linked to the electrical activity of the epithelium. Interestingly, the results seen with LPS have reinforced the importance of amiloride-sensitive $\mathrm{Na}^{+}$channels in mediating EpDRF-induced smooth muscle relaxation since LPS-induced changes in epithelial electrophysiology appear to be due alterations in these $\mathrm{Na}^{+}$channels. At the most fundamental level, apical $\mathrm{Na}^{+}$channels may control airway reactivity by modulating the synthesis, release, and/or effects of EpDRF. 


\section{Future Directions}

The two, separate studies contained within this project have provided the groundwork for a more thorough investigation of the airway epithelial bioelectric mechanisms associated with the effects of EpDRF in guinea-pig isolated trachea. In fact, this study has left many loose ends which could be tied up with future experiments.

First, the effects of other hypertonic solutions (i.e., $\mathrm{KCl}$, sucrose, urea, etc.), on epithelial bioelectric responses could be examined to determine if the epithelial response to hypertonic challenge is osmolyte dependent. In addition, the effects of ion channel and transporter inhibitors on the epithelium's bioelectric response to these hypertonic solutions could also be investigated for similar reasons. In addition, these experiments could be repeated with LPStreated tissue to determine how this would influence the epithelial bioelectric responses to hypertonic solution.

Since it appears that alterations in amiloride-sensitive $\mathrm{Na}^{+}$channels play a significant role in the hyperpolarization of the $\mathrm{V}_{\mathrm{ms}}$ following LPS-treatment, it would be interesting to examine these channels at the molecular level. Channel protein or mRNA levels may change after LPStreatment which could be examined by Western and Northern blotting, respectively. In addition, channel kinetics could be investigated through patch clamping. The effect of amiloride and the other ion channel and transporter inhibitors on LPS-induced alterations in EpDRF-induced smooth muscle relaxation could also be investigated. Finally, it would be interesting to see if epithelial depolarization is potentiated following LPS-treatment in response to the IL-application of D-mannitol, urea, and $\mathrm{KCl}$, which could shed more light on the relationship between epithelial depolarization and the degree of EpDRF-induced smooth muscle relaxation.

Although it was not a major focus of this study, it would be extremely interesting to distinguish which epithelial muscarinic receptor subtypes were responsible for the biphasic electrical responses to MCh, not only in saline-treated animals but also in those treated with LPS. The use of selective, muscarinic receptor antagonists could give insight into the receptor 
subtypes involved. In addition, it would be of interest to distinguish why LPS-treatment only affects the bioelectric reactivity of some muscarinic receptor subtypes and not others. This could be accomplished through an examination of receptor binding, coupling, expression, etc. 


\section{Literature Cited}

Allen BG and Walsh MP (1994) The biochemical basis of the regulation of smooth muscle contraction. Trends Biochem Sci 19:362-368.

Anderson SD (1985) Exercise-induced asthma: The state of the art. Chest 87:191S-195S.

Anderson SD and Daviskas E (1999) Airway drying and exercise-induced asthma, in ExerciseInduced Asthma (McFadden ER ed) pp 77-113, Marcel Dekker, Inc., New York.

Barnes PJ (1988) Airway neuropeptides, in Asthma: Basic Mechanisms and Clinical Management (Barnes PJ, Rodger IW and Thomson NC eds) pp 395-413, Academic Press, London.

Barnes PJ, Cuss TM and Palmer JB (1985) The effect of airway epithelium on smooth muscle contractility in bovine trachea. Br J Pharmacol 86:685-691.

Barnes PJ, Thomson NC and Rodger IW (1998) Preface, in Asthma: Basic Mechanisms and Clinical Management (Barnes PJ, Thomson NC and Rodger IW eds) Academic Press, London.

Bone RC (1996) The sepsis syndrome: Definition and general approach to management, in The Sepsis Syndrome (Dorinsky PM ed) pp 175-181, WB Saunders Company, Philadelphia.

Boucher RC (1999) Molecular insights into the physiology of the 'thin film' of airway surface liquid. J Physiol 516:631-638.

Boucher RC, Bromberg PA and Gatzy JT (1980) Airway transepithelial electric potential in vivo: Species and regional differences. J Appl Physiol 48:169-176.

Boucher RC, Narvarte J, Cotton C, Stutts MJ, Knowles MR, Finn AL and Gatzy JT (1982) Sodium absorption in mammalian airways, in Fluid and Electrolyte Abnormalities in Exocrine Glands in Cystic Fibrosis (Quinton PM, Martinez JR and Hopfer U eds) pp 271-287, San Francisco Press, San Francisco.

Bouhuys A (1977) The physiology of breathing, Grune \& Stratton, New York.

Bourke JR, Sand O, Abel KC, Huxham GJ and Manley SW (1995) Chloride channels in the apical membrane of thyroid epithelial cells are regulated by cAMP. J Endocrinol 147:441-448. 
Buxton ILO, Cheek DJ, Eckman D, Westfall DP, Sanders KM and Keef KD (1993) N ${ }^{\mathrm{G}}$-nitro Larginine methyl ester and other alkyl esters of arginine are muscarinic receptor antagonists. Circ Res 72:387-395.

Clarke LL and Boucher RC (1993) Ion and water transport across airway epithelia, in Pharmacology of the Respiratory Tract: Experimental and Clinical Research (Chung KF and Barnes PJ eds) pp 505-550, Marcel Dekker, Inc., New York.

Cockcroft DW and O'Byrne PM (1993) Mechanisms of airway hyperresponsiveness, in Bronchial Asthma: Mechanisms and Therapeutics (Weiss EB and Stein M eds) pp 32-42, Little, Brown and Company, Boston.

Cohen DM (1997) Mitogen-activated protein kinase cascades and the signaling of hyperosmotic stress to immediate early genes. Comp Biochem Physiol 117A:291-299.

Croxton TL (1993) Electrophysiological properties of guinea pig tracheal epithelium determined by cable analysis. Am J Physiol 265:L38-L44.

Curtis H and Barnes NS (1989) Biology of Organisms, Worth Publishers, Inc., New York.

Divertie MB and Brass A (1980) The Netter Collection of Medical Illustrations Vol. 7 Respiratory System, Novartis, East Hanover, N.J.

Dortch-Carnes J, Van Scott MR and Fedan JS (1999) Changes in smooth muscle tone during osmotic challenge in relation to epithelial bioelectric events in guinea-pig isolated trachea. J Pharmacol Exp Ther 289:911-917.

Fawcett DW and Jensh RP (1997) Bloom and Fawcett Concise Histology, Chapman \& Hall, New York.

Fedan JS and Frazer DG (1992) Influence of epithelium on the reactivity of guinea pig isolated, perfused trachea to bronchoactive drugs. J Pharmacol Exp Ther 262:741-750.

Fedan JS, Hay DWP, Farmer SG and Raeburn D (1988) Epithelial cells: Modulation of airway smooth muscle reactivity, in Asthma: Basic Mechanisms and Clinical Management (Barnes PJ, Rodger IW and Thomson NC eds) pp 143-162, Academic Press, London.

Fedan JS, Millecchia LL, Johnston RA, Rengasamy A, Hubbs A, Dey RD, Yuan L-X, Watson D, Goldsmith WT, Reynolds JS, Orsini L, Dortch-Carnes J, Cutler D and Frazer DG (2000) Effect of ozone treatment on airway reactivity and epithelium-derived relaxing factor in guinea pigs. J Pharmacol Exp Ther 293:724-734.

Fedan JS, Warner TE, Yuan L-X, Robinson VA and Frazer DG (1995) Nitric oxide synthase inhibitor and lipopolysaccharide effects on reactivity of guinea pig airways. $J$ Pharmacol Exp Ther 272:1141-1150. 
Fedan JS, Yuan L-X, Chang VC, Viola JO, Cutler D and Pettit LL (1999) Osmotic regulation of airway reactivity by the epithelium. J Pharmacol Exp Ther 289:901-910.

Fernandes LB and Goldie RG (1990) Pharmacological evaluation of a guinea-pig tracheal epithelium-derived inhibitory factor (EpDIF). Br J Pharmacol 100:614-618.

Fernandes LB, Preuss JMH, Paterson JW and Goldie RG (1990) Epithelium-derived inhibitory factor in human bronchus. Eur J Pharmacol 187:331-336.

Flavahan NA, Aarhus LL, Rimele TJ and Vanhoutte PM (1985) Respiratory epithelium inhibits bronchial smooth muscle tone. J Appl Physiol 58:834-838.

Folkerts G, Engels F and Nijkamp FP (1989) Endotoxin-induced hyperreactivity of the guineapig isolated trachea coincides with decreased prostaglandin $\mathrm{E}_{2}$ production by the epithelial layer. Br J Pharmacol 96:388-394.

Folkerts G, Henricks PAJ, Slootweg PJ and Nijkamp FP (1988) Endotoxin-induced inflammation and injury of the guinea pig respiratory airways cause bronchial hyporeactivity. Am Rev Respir Dis 137:1441-1448.

Folkerts G and Nijkamp FP (1998) Airway epithelium: More than just a barrier! Trends Pharmacol Sci 19:334-341.

Freed AN (1995) Models and mechanisms of exercise-induced asthma. Eur Respir J 8:17701785.

Freed AN and Croxton TL (1993) Submucosal tissues modulate the bioelectric properties of airway epithelium. Am J Respir Cell Mol Biol 8:433-438.

Frizzell RA, Field M and Schultz SG (1979) Sodium-coupled chloride transport by epithelial tissues. Am J Physiol 236:F1-F8.

Goldie RG and Hay DWP (1997) Epithelium-dependent responsiveness of airway smooth muscle: The role of epithelium-derived relxant factors, in Asthma (Barnes PJ, Grunstein MM, Leff AR and Woolcock AJ eds) pp 901-915, Lippincott-Raven, Philadelphia.

Gross NJ (1988) Cholinergic control, in Asthma: Basic Mechanisms and Clinical Management (Barnes PJ, Rodger IW and Thomson NC eds) pp 381-393, Academic Press, London.

Güc MO, Ilhan M and Kayaalp O (1988a) Epithelium-dependent relaxation of guinea-pig tracheal smooth muscle by carbachol. Arch Int Pharmacodyn 294:241-247.

Güc MO, Ilhan M and Kayaalp SO (1988b) The rat anococcygeus muscle is a convenient bioassay organ for the airway epithelium-derived relaxant factor. Eur J Pharmacol 148:405-409. 
Haüssinger D (1996) The role of cellular hydration in the regulation of cell function. Biochem $J$ 313:697-710.

Hay DWP, Farmer SG, Raeburn D, Robinson VA, Fleming WW and Fedan JS (1986) Airway epithelium modulates the reactivity of guinea-pig respiratory smooth muscle. Eur $J$ Pharmacol 129:11-18.

Hoang LM, Chen C and Mathers DA (1997) Lipopolysaccharide rapidly activates $\mathrm{K}^{+}$channels at the intracellular membrane face of rat cerebral artery smooth muscle cells. Neurosci Lett 231:25-28.

Holroyde MC (1986) The influence of epithelium on the responsiveness of guinea-pig isolated trachea. Br J Pharmacol 87:501-507.

Horowitz A, Menice CB, Laporte R and Morgan KG (1996) Mechanisms of smooth muscle contraction. Physiol Rev 76:967-1003.

Hulsmann AR and Jongste JCD (1996) Modulation of airway responsiveness by the airway epithelium in humans: Putative mechanisms. Clin Exp Allergy 26:1236-1242.

Ilhan M and Sahin I (1986) Tracheal epithelium releases a vascular smooth muscle relaxant factor: Demonstrated by bioassay. Eur J Pharmacol 131:293-296.

Ind PW and Barnes PJ (1988) Adrenergic control of airways in asthma, in Asthma: Basic Mechanisms and Clinical Mangement (Barnes PJ, Rodger IW and Thomson NC eds) pp 357-393, Academic Press, London.

Ito Y, Mizuno Y, Aoyama M, Kume H and Yamaki K (2000) CFTR-mediated anion conductance regulates $\mathrm{Na}^{+}-\mathrm{K}^{+}$-pump activity in Calu-3 human airway cells. Biochem Biophys Res Comm 274:230-235.

Janssen LJ, Daniel EE and Rodger IW (1998) Airway smooth muscle cells: Structure and function, in Asthma: Basic Mechanisms and Clinical Management (Barnes PJ, Rodger IW and Thomson NC eds) pp 90-112, Academic Press, London.

Jeffrey PK (1998) Airway pathology in asthma, in Asthma: Basic Mechanisms and Clinical Management (Barnes PJ, Rodger IW and Thomson NC eds) pp 47-64, Academic Press, London.

Johnson LR (1997) Essential Medical Physiology (1997) Lippincott-Raven Publishers, Philadelphia.

Johnston RA and Fedan JS (1999) Effects of lipopolysaccharide on tracheal epithelial bioelectric responses to serosally- and mucosally-applied methacholine. Am J Respir Crit Care Med 159:A294. 
Jongejan RC, Jongste JCD, Raatgeep RC, Bonta IL and Kerrebijn KF (1990) Effects of changes in osmolarity on isolated human airways. J Appl Physiol 68:1568-1575.

Kagawa J and Kerr HD (1970) Effects of brief graded exercise on specific airway conductance in normal subjects. $J$ Appl Physiol 28:138-144.

Kamosinska B, Radomski MW, Duszyk M, Radomski A and Man SF (1997) Nitric oxide activates chloride currents in human lung epithelial cells. Am J Physiol 272:L1098-1104.

Knowles MR, Olivier K, Noone P and Boucher RC (1995) Pharmacologic modulation of salt and water in the airway epithelium in cystic fibrosis. Am J Respir Crit Care Med 151:S65S69.

Kotlikoff MI and Kamm KE (1996) Molecular mechanisms of \$-adrenergic relaxation of airway smooth muscle. Аnпu Rev Physiol 58:115-141.

Kreydiyyeh SI (2000) Cyclic AMP and furosemide stimulate the $\mathrm{Na}^{+}-\mathrm{K}^{+}$ATPase in isolated rat jejunal cells. Pharmcol Res 41:179-185.

Laitinen LA, Heino M, Laitinen A, Kava T and Haahtela T (1985) Damage of the airway epithelium and bronchial reactivity in patients with asthma. Am Rev Resp Dis 131:599606.

Lamb F, Kooy N and Lewis S (2000) Role of $\mathrm{Cl}^{!}$channels in alpha-adrenoceptor-mediated vasoconstriction in the anesthetized rat. Eur J Pharmacol 401:403-412.

Lang F, Busch GL, Ritter M, Volkl H, Waldegger S, Gulbins E and Haüssinger D (1998)

Functional significance of cell volume regulatory mechanisms. Physiol Rev 70:254-306.

Lawrence TE, Frazer DG and Fedan JS (1996) Hyporeactivity to methacholine in vitro accompanying hyperreactivity to methacholine in vivo in a guinea-pig model of asthma. Am J Respir Crit Care Med 153:A627.

Lehninger AL, Nelson DL and Cox MM (1993) Principles of Biochemistry, Worth Publishers, New York.

Levitzky MG (1995) Pulmonary Physiology, McGraw-Hill, Inc., New York.

Lomask K (1996) BioSystem XA User's Manual Version 0.9.3, Buxco Electronics, Inc., Sharon, CT.

Lüderitz O, Freudenberg MA, Galanos C, Lehmann V, Rietschel ET and Shaw DH (1982) Lipopolysaccharides of gram-negative bacteria, in Current Topics in Membranes and Transport (Bronner F and Kleinzeller A eds) pp 79-151, Academic Press, London. 
Mak JCW and Barnes PJ (1990) Autoradiographic visualization of muscarinic receptor subtypes in human and guinea pig lung. Am Rev Respir Dis 141:1559-1568.

Marsh CB and Wewers M (1996) The pathogenesis of sepsis: Factors that modulate the response to gram-negative bacterial infection, in The Sepsis Syndrome (Dorinsky PM ed) pp 183-197, WB Saunders Company, Philadelphia.

McFadden ER (1995) Exercise-induced airway obstruction, in Asthma (Martin RJ ed) pp 671682, WB Saunders Company, Philadelphia.

McFeely JE and Hudson LD (1994) Sepsis, multiple-organ dysfunction syndrome, and adult respiratory distress syndrome in humans, in Endotoxin and the Lungs, (Brigham KL ed) pp 321-350, Marcel Dekker, Inc., New York.

McManus ML, Churchwell KB and Strange K (1995) Regulation of cell volume in health and disease. $N$ Engl J Med 333:1260-1266.

Miyoshi H and Nakaya Y (1994) Endotoxin-induced nonendothelial nitric oxide activates the $\mathrm{Ca}^{2+}$-activated $\mathrm{K}^{+}$channel in cultured vascular smooth muscle cells. J Mol Cell Cardiol 26:1487-1495.

Morrison KJ, Gao Y and Vanhoutte PM (1990) Epithelial modulation of airway smooth muscle. Am J Physiol 258:L254-L262.

Munakata M, Huang I, Mitzner W and Menkes H (1989) Protective role of epithelium in the guinea pig airway. $J$ Appl Physiol 66:1547-1552.

Munakata M, Masaki Y, Sakuma I, Ukita H, Otsuka Y, Homma Y and Kawakami Y (1990) Pharmacological differentiation of epithelium-derived relaxing factor from nitric oxide. $J$ Appl Physiol 69:665-670.

Munakata M, Mitzner W and Menkes H (1988) Osmotic stimuli induce epithelial-dependent relaxation in the guinea pig trachea. $J$ Appl Physiol 64:466-471.

Murlas C (1986) Effects of mucosal removal on guinea-pig airway smooth muscle responsiveness. Clin Sci 70:571-575.

Myers TP, Myers PR, Adams HR and Parker JL (1999) Enhanced prostanoid-mediated vasorelaxation in pulmonary arteries isolated during experimental endotoxemia. Shock 11:436-442.

Nagaishi C, Nagasawa N, Yamashita M, Okada Y and Inaba N (1972) Functional anatomy and histology of the lung, University Park Press, Baltimore. 
Oosterhout AJMV, Nijnanten FMAW-V and Nijkamp FP (1991) Effect of cyclosporin-A treatment on endotoxin-induced airway hyperreactivity in vivo and in vitro in guinea pigs. Naunyn-Schmideberg's Arch Pharmcol 343:542-545.

Parker JC (1993) In defense of cell volume? Am J Physiol 265:C1191-C1200.

Raeburn D and Fedan JS (1989) The effects of alterations in electrogenic $\mathrm{Na}^{+} / \mathrm{K}^{+}$-pumping in guinea-pig isolated trachealis: Their modulation by the epithelium. Br J Pharmacol 98:343-350.

Raeburn D, Hay DWP, Farmer SG and Fedan JS (1986a) Epithelium removal increases the reactivity of human isolated tracheal muscle to methacholine and reduces the effect of verapamil. Eur J Pharmacol 123:451-453.

Raeburn D, Hay DWP, Robinson VA, Farmer SG, Fleming WW and Fedan JS (1986b) The effect of verapamil is reduced in isolated airway smooth muscle preparations lacking the epithelium. Life Sci 38:809-816.

Raeburn D, Sequeira DJ and Backes WL (1988) Possible invovlement of cytochrome P-450 in the epithelium-modulated response to methacholine in guinea pig trachea. Eur $J$ Pharmacol 123:451-453.

Raetz CRH (1996) Bacterial lipopolysaccharides: A remarkable family of bioactive macroamphiphiles, in Escherichia coli and Salmonella: Cellular and molecular biology (Neidhardt FC ed) pp 1035-1063, ASM Press, Washington, D.C.

Rietschel ET, Brade H, Holst O, Brade L, Müller-Loennies S, Mamat U, Zähringer U, Beckmann F, Seydel U, Brandenburg K, Ulmer AJ, Mattern T, Heine H, Schletter J, Loppnow H, Schönbeck U, Flad H-D, Hauschildt S, Schade UF, Padova FD, Kusumoto S and Schumann RR (1996) Bacterial endotoxin: Chemical composition, biological recognition, host response, and immunological detoxification, in Pathology of Septic Shock (Rietschel ET and Wagner H eds) pp 40-81, Springer, Berlin.

Rietschel ET, Kirikae T, Schade FU, Mamat U, Schmidt G, Loppnow H, Ulmer AJ, Zähringer U, Seydel U, Padova FD, Schreier M and Brade H (1994) Bacterial endotoxin: Molecular relationships of structure to activity and function. FASEB J 8:217-225.

Robison TW and Kim K-J (1994) Air-interface cultures of guinea pig airway epithelial cells: Effects of active sodium and chloride transport inhibitors on bioelectric properties. Exp Lung Res 20:101-117.

Roux E, Molimard M, Savineau J-P and Marthan R (1998) Muscarinic stimulation of airway smooth muscle cells. Gen Pharmacol 31:349-356. 
Saunders KK, Cates JA and Roslyn JJ (1993) Prostaglandin $E_{2}$ stimulates ion transport in prairie dog gallbladder. Digest Dis Sci 38:167-72.

Sessler CN, Bloomfield GL and Fowler AA (1996) Current concepts of sepsis and acute lung injury, in The Sepsis Syndrome (Dorinsky PM ed) pp 213-235, WB Saunders Company, Philadelphia.

Shelhamer JH, Levine SJ, Wu T, Jacoby DB, Kaliner MA and Rennard SI (1995) Airway inflammation. Ann Intern Med 123:288-304.

Sherwood L (1997) Human Physiology: From Cells to Systems, Wadsworth Publishing Company, Belmont, CA.

Somlyo AP and Somlyo AV (1994) Signal transduction and regulation in smooth muscle. Nature 372:231-236.

Soybel DI (1994) Applications of electrophysiology in studies of ion transport by gut mucosa. $J$ Surg Res 57:510-526.

Stein B, Frank P, Schmitz W, Scholz H and Thoenes M (1996) Endotoxin and cytokines induce direct cardiodepressive effects in mammalian cardiomyocytes via induction of nitric oxide synthase. J Mol Cell Cardiol 28:1631-1639.

Stutts MJ and Bromberg PA (1987) Effects of ozone on airway epithelial permeability and ion transport. Toxicol Lett 35:315-319.

Suzuki K, Kawahara K, Terada N, Nomura T, Konno A and Fukuda Y (1999) Effect of ion transport inhibitors and methacholine on short-circuit current of isolated guinea pig nasal epithelium. Jap J Physiol 49:99-106.

Tamai H and Gaginella TS (1993) Direct evidence for nitric oxide stimulation of electrolyte secretion in the rat colon. Free Radical Res Comm 19:229-239.

Tamaoki J, Chiyotani A, Tagaya E, Takemura H and Konno K (1996) Cholinergic control of rabbit tracheal transepithelial potential difference in vivo. Eur Respir J 9:1632-1636.

Tamaoki J, Isono K, Chiyotani A, Kondo M and Konno K (1992a) Angiotensin II-1 receptormediated $\mathrm{Cl}^{\text {! }}$ secretion by canine tracheal epithelium. Am Rev Resp Dis 146:1187-1191.

Tamaoki J, Kanemura T, Sakai N, Isono K, Chiyotani A, Yamauchi F, Takizawa T and Konno K (1992b) Prostaglandin $\mathrm{D}_{2}$ increases $\mathrm{Cl}^{\text {! }}$ secretion across canine tracheal epithelium through cyclo-oxygenase stimulation and cAMP production. Acta Physiol Scand 145:1-6.

Tamaoki J, Tagaya E, Isono K, Kondo M and Konno K (1997) Role of $\mathrm{Ca}^{2+}$-activated K channel in epithelium-dependent relaxation of human bronchial smooth muscle. $\mathrm{Br} J$ Pharmacol 121:794-798. 
Teeter J, Munakata M and Mitzner W (1988) Attenuation of epithelial dependent relaxation in guinea pig trachea by gossypol. Am Rev Resp Dis 137:A322.

Weibel ER (1963) Morphometry of the human lung, Academic Press, London.

West JB (1995) Respiratory Physiology-the essentials, Williams \& Wilkins, Baltimore.

Widdicombe JH (1991) Physiology of airway epithelia, in The Airway Epithelium: Physiology, Pathophysiology, and Pharmacology (Farmer SG and Hay DWP eds) pp 41-64, Marcel Dekker, Inc., New York.

Widdicombe JH and Widdicombe JG (1995) Regulation of human airway surface liquid. Resp Physiol 99:3-12.

Wilkinson MF, Earle ML, Triggle CR and Barnes SA (1996) Interleukin-1beta, tumour necrosis factor-alpha, and LPS enhance calcium channel current in isolated vascular smooth muscle cells of rat artery. FASEB J 10:785-791.

Willumsen NJ, Davis CW and Boucher RC (1994) Selective response of human airway epithelia to luminal but not serosal solution hypertonicity. J Clin Invest 94:779-787.

Yamawaki I, Tamaoki J, Kanemura T, Horii S and Takizawa T (1990) Effects of lipopolysaccharide from Pseudomonas aeruginosa on airway smooth muscle function in guinea pigs. Respiration 57:268-274. 